\title{
Review \\ Dithiocarbamate Complexes of Platinum Group Metals: Structural Aspects and Applications
}

\author{
Yee Seng Tan ${ }^{1, *}$, Chien Ing Yeo ${ }^{1} \mathbb{D}$, Edward R. T. Tiekink ${ }^{1}\left(\mathbb{D}\right.$ and Peter J. Heard ${ }^{2, *} \mathbb{C}$ \\ 1 Research Centre for Crystalline Materials, School of Medical and Life Sciences, Sunway University, \\ Bandar Sunway 47500, Selangor Darul Ehsan, Malaysia; allyy@sunway.edu.my (C.I.Y.); \\ edwardt@sunway.edu.my (E.R.T.T.) \\ 2 Chancellery Office, Sunway University, Bandar Sunway 47500, Selangor Darul Ehsan, Malaysia \\ * Correspondence: yeesengt@sunway.edu.my (Y.S.T.); pheard@sunway.edu.my (P.J.H.)
}

check for updates

Citation: Tan, Y.S.; Yeo, C.I.;

Tiekink, E.R.T.; Heard, P.J.

Dithiocarbamate Complexes of Platinum Group Metals: Structural Aspects and Applications. Inorganics 2021, 9, 60. https://doi.org/10.3390/ inorganics 9080060

Academic Editor: Graeme Hogarth

Received: 29 June 2021

Accepted: 19 July 2021

Published: 22 July 2021

Publisher's Note: MDPI stays neutral with regard to jurisdictional claims in published maps and institutional affiliations.

Copyright: (c) 2021 by the authors. Licensee MDPI, Basel, Switzerland. This article is an open access article distributed under the terms and conditions of the Creative Commons Attribution (CC BY) license (https:/ / creativecommons.org/licenses/by/ $4.0 /)$.

\begin{abstract}
The incorporation of dithiocarbamate ligands in the preparation of metal complexes is largely prompted by the versatility of this molecule. Fascinating coordination chemistry can be obtained from the study of such metal complexes ranging from their preparation, the solid-state properties, solution behavior as well as their applications as bioactive materials and luminescent compounds, to name a few. In this overview, the dithiocarbamate complexes of platinum-group elements form the focus of the discussion. The structural aspects of these complexes will be discussed based upon the intriguing findings obtained from their solid- (crystallographic) and solution-state (NMR) studies. At the end of this review, the applications of platinum-group metal complexes will be discussed.
\end{abstract}

Keywords: platinum-group metals; dithiocarbamate; metal dithiocarbamate; X-ray crystallography; NMR; antitumor; CVD; sensors

\section{Introduction}

Dithiocarbamates $\left(\mathrm{RR}^{\prime} \mathrm{NCS}_{2}{ }^{-}\right.$; Figure 1) are highly versatile ligands that have attracted the attention of coordination chemists for decades [1-5]. These, usually, mono-anionic species normally coordinate in an essentially symmetric fashion via the two sulfur atoms and are able to form stable complexes with a remarkable range of different metals in a variety of different oxidation states, including those that are higher than normal. This being stated, bridging modes of coordination are sometimes observed in the structural chemistry of the main group elements [6-9]. It is also noted that polyfunctional dithiocarbamate ligands, that is, bearing more than one $\mathrm{CS}_{2}{ }^{-}$residue, are known although comparatively rare [10] as are multifunctional ligands incorporating $\mathrm{CS}_{2}{ }^{-}$functionality as well as other coordinating sites, for example, pyridyl donors [11]. The latter two classes of ligand are invariably bridging and lead to various multi-metallic aggregates.

$$
{ }_{S}^{-S}{ }_{S^{\prime}}^{\mathrm{R}^{\prime}}
$$

I

Figure 1. Canonical forms of dithiocarbamate, $\mathrm{RR}^{\prime} \mathrm{NCS}_{2}{ }^{-}, \mathrm{R} / \mathrm{R}^{\prime}=\mathrm{H}$, alkyl and aryl.

The versatility of dithiocarbamate ligands stems from the near equal contributions of the two resonance forms, I and III $(2 \times 30 \%)$ and II $(40 \%)$ (Figure 1$)$. This is also significant in terms of supramolecular chemistry as the resulting $\mathrm{MS}_{2} \mathrm{C}$ chelate ring has significant $\pi$-character, metallo-aromaticity [12], and can participate as an acceptor in $\mathrm{C}-\mathrm{H} \cdots \pi$ (chelate 
ring) interactions in the same way as $\mathrm{C}-\mathrm{H} \cdots \pi$ (arene) interactions $[13,14]$ but impart greater energies of stabilization [15-17].

From a structural point of view, dithiocarbamate complexes are of interest precisely because of the range of different heavy element ions to which they can coordinate and the resulting structural motifs. In summary, recent work has uncovered an even more diverse molecular and supramolecular chemistry with a wide variety of structural arrangements.

However, dithiocarbamate complexes are not just of intrinsic interest. Dithiocarbamates themselves are biologically active and when coupled with different metals, transition metals and main group elements, which often show enhanced activity, this activity can be modified, fine-tuned, and amplified [18-22]. Dithiocarbamate complexes also show utility as single-source precursors to metal sulfide nanoparticles [23,24]. It is thus unsurprising there has been no let-up in recent years in the number of dithiocarbamate complexes reported in the literature. In this review, we explore recent advances in the molecular chemistry of platinum-group metal dithiocarbamate complexes, focusing on structural data acquired via X-ray crystallography and multi-NMR spectroscopy as well as an overview of potential applications. In many of these complexes, the dithiocarbamate ligands have been incorporated because of their ability to stabilize the metal moiety, allowing other, active functional groups to be present. In other cases, it is the dithiocarbamates themselves that are the primary focus of interest. In either case, without the presence of such a stable and tolerant ligand as dithiocarbamate, the potential utility of the resultant complexes is likely to be diminished.

Homoleptic dithiocarbamate complexes are typically prepared in a single-pot synthesis from the reaction of a secondary (or, less commonly, primary) amine with carbon disulfide under basic conditions and in the presence of the appropriate metal ion synthon (Equation (1)) [25]. Alternative routes, more applicable to heteroleptic complexes include the reaction of a suitable dithiocarbamate salt (typically the ammonium [26], Group 1 [27] salt) with a metal halide (Equation (2)), or insertion of $\mathrm{CS}_{2}$ into the metal-nitrogen bond of the corresponding amide complex (Equation (3)) [28].

$$
\begin{gathered}
\mathrm{nNHR}_{2}+\mathrm{nCS}_{2}+\mathrm{M}^{\mathrm{n}+} \stackrel{\mathrm{OH}^{-}}{\longrightarrow}\left(\mathrm{S}_{2} \mathrm{CNR}_{2}\right)_{\mathrm{n}}+\mathrm{H}_{2} \mathrm{O} \\
\mathrm{nX}\left(\mathrm{S}_{2} \mathrm{CNR}_{2}\right)+\mathrm{M}^{\mathrm{n}+} \mathrm{Y}_{\mathrm{n}} \rightarrow \mathrm{M}\left(\mathrm{S}_{2} \mathrm{CNR}_{2}\right)_{\mathrm{n}}+\mathrm{nXY} \\
\mathrm{M}\left(\mathrm{NR}_{2}\right)_{\mathrm{n}}(\mathrm{L})+\mathrm{nCS}_{2} \rightarrow \mathrm{M}\left(\mathrm{S}_{2} \mathrm{CNR}_{2}\right)_{\mathrm{n}}(\mathrm{L})
\end{gathered}
$$

$\mathrm{R}=$ alkyl/aryl; $\mathrm{M}=$ metal; $\mathrm{X}=$ cation; $\mathrm{Y}=$ halides; $\mathrm{L}=$ ancillary ligand.

\section{Methods}

A systematic search of the Cambridge Structural Database (CSD, version 5.42) [29] employing ConQuest (version 2.0.4) [30] was performed for the present bibliographic review of the crystallographic literature where the search evaluated all platinum-group metals $\left(\mathrm{Ru}, \mathrm{Rh}, \mathrm{Pd}, \mathrm{Os}\right.$, Ir, and $\mathrm{Pt}$ ) structures bearing a $\mathrm{NCS}_{2}$ moiety. The search was restricted to structures deposited from 2004 onwards. No other restrictions were applied. All crystallographic diagrams were generated employing DIAMOND [31] using the Crystallographic Information Files deposited for each structure.

\section{Results}

The solid-state structures revealed by X-ray crystallographic methods will be discussed initially. This will be followed by an evaluation of solution-state structures/chemistry established by multi-NMR studies as well as an overview of complementary solid-state NMR studies. Lastly, comments will be made on the potential applications of the platinum group metal dithiocarbamate complexes. 


\subsection{Solid-State Structures}

The discussion of the platinum group metal dithiocarbamate structures is made in the order following the Group of the Periodic Table, followed by the classification of the complexes such as binary, ternary, etc., and lastly, as organometallic species. A general overview of molecular structures will be provided and molecular packing only described for examples showing intriguing interactions. The dithiocarbamate ligands employed in this review are shown in Table 1 and Figure 2. The full chemical composition for the crystals discussed is included in the respective table under each subsection.

Table 1. List of dithiocarbamates $\mathrm{L}^{1}$ to $\mathrm{L}^{56}$.

\begin{tabular}{|c|c|c|}
\hline \multicolumn{3}{|c|}{${ }^{-} \mathrm{S}_{\mathbf{2}} \mathrm{CNRR}^{\prime}$} \\
\hline Ligand & $\mathbf{R}$ & $\mathbf{R}^{\prime}$ \\
\hline $\mathrm{L}^{1}$ & $\mathrm{H}$ & $\mathrm{H}$ \\
\hline $\mathrm{L}^{2}$ & $\mathrm{H}$ & $\mathrm{Me}$ \\
\hline $\mathrm{L}^{3}$ & $\mathrm{H}$ & Et \\
\hline $\mathrm{L}^{4}$ & $\mathrm{H}$ & $\mathrm{Ph}$ \\
\hline $\mathrm{L}^{5}$ & $\mathrm{H}$ & p-tolyl \\
\hline $\mathrm{L}^{6}$ & $\mathrm{H}$ & $\mathrm{C}_{6} \mathrm{H}_{4} \mathrm{Et}-\mathrm{p}$ \\
\hline $\mathrm{L}^{7}$ & $\mathrm{H}$ & $\mathrm{C}_{6} \mathrm{H}_{4} \mathrm{Cl}-\mathrm{p}$ \\
\hline $\mathrm{L}^{8}$ & $\mathrm{Me}$ & $\mathrm{Me}$ \\
\hline $\mathrm{L}^{9}$ & $\mathrm{Me}$ & $\mathrm{CH}_{2} \mathrm{C} \equiv \mathrm{CH}$ \\
\hline $\mathrm{L}^{10}$ & $\mathrm{Me}$ & $\mathrm{CH}_{2} \mathrm{CH}=\mathrm{CH}_{2}$ \\
\hline $\mathrm{L}^{11}$ & $\mathrm{Me}$ & $\mathrm{n}-\mathrm{Bu}$ \\
\hline $\mathrm{L}^{12}$ & $\mathrm{Me}$ & n-Hex \\
\hline $\mathrm{L}^{13}$ & $\mathrm{Me}$ & $\mathrm{CH}_{2} \mathrm{Ph}$ \\
\hline $\mathrm{L}^{14}$ & $\mathrm{Me}$ & $\mathrm{Ph}$ \\
\hline $\mathrm{L}^{15}$ & $\mathrm{Me}$ & Cy \\
\hline $\mathrm{L}^{16}$ & $\mathrm{Me}$ & $\mathrm{CH}_{2} \mathrm{CH}(\mathrm{OMe})_{2}$ \\
\hline $\mathrm{L}^{17}$ & $\mathrm{Me}$ & $\mathrm{CH}_{2} \mathrm{COO}^{-}$ \\
\hline $\mathrm{L}^{18}$ & $\mathrm{Me}$ & $\mathrm{CH}_{2} \mathrm{COOH}$ \\
\hline $\mathrm{L}^{19}$ & $\mathrm{Me}$ & $\mathrm{CH}_{2} \mathrm{COOMe}$ \\
\hline $\mathrm{L}^{20}$ & $\mathrm{Me}$ & $\mathrm{CH}_{2} \mathrm{COOEt}$ \\
\hline $\mathrm{L}^{21}$ & $\mathrm{Me}$ & $\mathrm{CH}_{2} \mathrm{COO}-t-\mathrm{Bu}$ \\
\hline $\mathrm{L}^{22}$ & $\mathrm{Me}$ & $\mathrm{CH}_{2} \mathrm{CH}_{2} \mathrm{OC}(=\mathrm{O}) \mathrm{CH}=\mathrm{CHCOOEt}$ \\
\hline $\mathrm{L}^{23}$ & $\mathrm{Me}$ & 2-methyl-1,3-dioxolane \\
\hline $\mathrm{L}^{24}$ & Et & Et \\
\hline $\mathrm{L}^{25}$ & Et & $\mathrm{CH}_{2} \mathrm{CH}_{2} \mathrm{OH}$ \\
\hline $\mathrm{L}^{26}$ & $\mathrm{CH}_{2} \mathrm{CH}_{2} \mathrm{OMe}$ & $\mathrm{CH}_{2} \mathrm{CH}_{2} \mathrm{OMe}$ \\
\hline $\mathrm{L}^{27}$ & $\mathrm{CH}_{2} \mathrm{CH}=\mathrm{CH}_{2}$ & $\mathrm{CH}_{2} \mathrm{CH}=\mathrm{CH}_{2}$ \\
\hline $\mathrm{L}^{28}$ & $\mathrm{n}-\operatorname{Pr}$ & $\mathrm{n}-\operatorname{Pr}$ \\
\hline $\mathrm{L}^{29}$ & i-Pr & i-Pr \\
\hline $\mathrm{L}^{30}$ & $\left(\mathrm{CH}_{2}\right)_{3} \mathrm{NMe}_{2}$ & $\left(\mathrm{CH}_{2}\right)_{3} \mathrm{NMe}_{2}$ \\
\hline $\mathrm{L}^{31}$ & $\mathrm{n}-\mathrm{Bu}$ & $\mathrm{n}-\mathrm{Bu}$ \\
\hline $\mathrm{L}^{32}$ & $\mathrm{n}-\mathrm{Bu}$ & $\mathrm{CH}_{2} \mathrm{Ph}$ \\
\hline $\mathrm{L}^{33}$ & $\mathrm{n}-\mathrm{Bu}$ & $\mathrm{Ph}$ \\
\hline $\mathrm{L}^{34}$ & $\mathrm{CH}_{2}(\mathrm{i}-\mathrm{Pr})$ & $\mathrm{CH}_{2}(\mathrm{i}-\mathrm{Pr})$ \\
\hline $\mathrm{L}^{35}$ & $\mathrm{n}-\mathrm{Hex}$ & $\mathrm{n}-\mathrm{Hex}$ \\
\hline $\mathrm{L}^{36}$ & $\mathrm{n}$-Dec & $\mathrm{n}-\mathrm{Dec}$ \\
\hline $\mathrm{L}^{37}$ & $\mathrm{CH}_{2} \mathrm{CH}(\mathrm{Me})(\mathrm{Et})$ & $\mathrm{CH}_{2} \mathrm{CH}(\mathrm{Me})(\mathrm{Et})$ \\
\hline $\mathrm{L}^{38}$ & $\mathrm{CH}_{2} \mathrm{CH}(\mathrm{Et})(\mathrm{n}-\mathrm{Bu})$ & $\mathrm{CH}_{2} \mathrm{CH}(\mathrm{Et})(\mathrm{n}-\mathrm{Bu})$ \\
\hline $\mathrm{L}^{39}$ & $\mathrm{CH}_{2} \mathrm{Ph}$ & $\mathrm{CH}_{2} \mathrm{Ph}$ \\
\hline $\mathrm{L}^{40}$ & $\mathrm{CH}_{2} \mathrm{Ph}$ & $\mathrm{CH}_{2} \mathrm{C}_{6} \mathrm{H}_{4} \mathrm{OMe}-3$ \\
\hline $\mathrm{L}^{41}$ & $\mathrm{CH}_{2} \mathrm{Ph}$ & $\mathrm{CH}_{2} \mathrm{C}_{5} \mathrm{H}_{4} \mathrm{~N}-3$ \\
\hline $\mathrm{L}^{42}$ & $\mathrm{CH}_{2} \mathrm{Ph}$ & $\mathrm{CH}_{2} \mathrm{C}_{4} \mathrm{H}_{3} \mathrm{NMe}$ \\
\hline
\end{tabular}


Table 1. Cont.

\begin{tabular}{|c|c|c|}
\hline \multicolumn{3}{|c|}{${ }^{-} \mathrm{S}_{\mathbf{2}} \mathrm{CNRR}^{\prime}$} \\
\hline Ligand & $\mathbf{R}$ & $\mathbf{R}^{\prime}$ \\
\hline $\mathrm{L}^{43}$ & $\mathrm{CH}_{2} \mathrm{Ph}$ & $\mathrm{CH}_{2} \mathrm{C}_{5} \mathrm{H}_{4} \mathrm{FeCp}$ \\
\hline $\mathrm{L}^{44}$ & $\left(\mathrm{CH}_{2}\right)_{2} \mathrm{PPh}_{2}$ & $\left(\mathrm{CH}_{2}\right)_{2} \mathrm{PPh}_{2}$ \\
\hline $\mathrm{L}^{45}$ & $\mathrm{C}_{5} \mathrm{H}_{4} \mathrm{~N}-2$ & $\mathrm{C}_{5} \mathrm{H}_{4} \mathrm{~N}-2$ \\
\hline $\mathrm{L}^{46}$ & $\mathrm{Ph}$ & $\mathrm{Ph}$ \\
\hline $\mathrm{L}^{47}$ & Cy & $\mathrm{Cy}$ \\
\hline $\mathrm{L}^{48}$ & p-tolyl & p-tolyl \\
\hline $\mathrm{L}^{49}$ & $\mathrm{C}_{6} \mathrm{H}_{4} \mathrm{CF}_{3}-\mathrm{p}$ & $\mathrm{C}_{6} \mathrm{H}_{4} \mathrm{CF}_{3}-\mathrm{p}$ \\
\hline $\mathrm{L}^{50}$ & $\mathrm{C}_{6} \mathrm{H}_{4}(\mathrm{t}-\mathrm{Bu})-\mathrm{p}$ & $\mathrm{C}_{6} \mathrm{H}_{4}(\mathrm{t}-\mathrm{Bu})-\mathrm{p}$ \\
\hline $\mathrm{L}^{51}$ & $\mathrm{CH}_{2} \mathrm{COO}^{-}$ & $\mathrm{CH}_{2} \mathrm{COO}^{-}$ \\
\hline $\mathrm{L}^{52}$ & $\mathrm{CH}_{2} \mathrm{COOH}$ & $\mathrm{CH}_{2} \mathrm{COOH}$ \\
\hline $\mathrm{L}^{53}$ & $\mathrm{CH}_{2} \mathrm{COOH}$ & $\mathrm{CH}_{2} \mathrm{COO}^{-}$ \\
\hline $\mathrm{L}^{54}$ & $\mathrm{CH}_{2} \mathrm{C}_{4} \mathrm{H}_{3} \mathrm{O}$ & $\mathrm{CH}_{2} \mathrm{C}_{4} \mathrm{H}_{3} \mathrm{O}$ \\
\hline $\mathrm{L}^{55}$ & $\mathrm{CH}_{2} \mathrm{C}_{6} \mathrm{H}_{4} \mathrm{OH}-\mathrm{O}$ & p-tolyl \\
\hline $\mathrm{L}^{56}$ & $\mathrm{CH}_{2} \mathrm{C}(=\mathrm{O}) \mathrm{N}(\mathrm{H})\left(\mathrm{CH}_{2}\right)_{3} \mathrm{CH}_{3}$ & $\mathrm{CH}_{2} \mathrm{C}(=\mathrm{O}) \mathrm{N}(\mathrm{H})\left(\mathrm{CH}_{2}\right)_{3} \mathrm{CH}_{3}$ \\
\hline
\end{tabular}

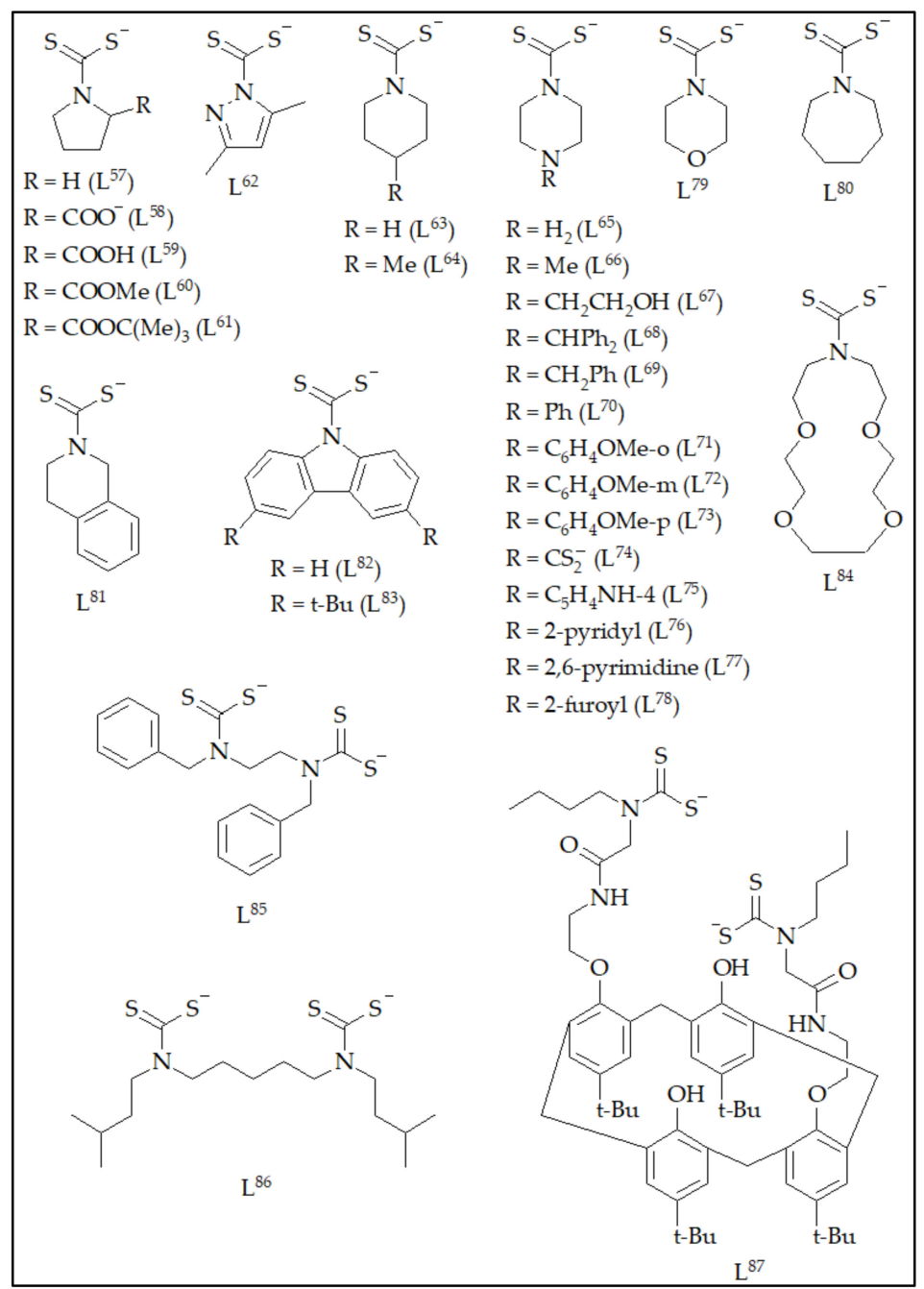

Figure 2. Chemical diagrams of dithiocarbamates $\mathrm{L}^{57}$ to $\mathrm{L}^{87}$.

\subsubsection{Ruthenium}

The CSD search for ruthenium complexes bearing a dithiocarbamate ligand consistent with the search parameters described in Section 2 resulted in 72 different crystal structures: 
these are listed in Table 2. The most common oxidation states of ruthenium complexes are +II and +III with ruthenium(II) complexes being the most predominant. Generally, the ruthenium complexes adopt octahedral coordination geometries with the common feature of the dithiocarbamate acting as a $\kappa^{2}$ ligand. Binary complexes $\mathbf{1}-\mathbf{5}$ feature ruthenium(III) centers while ternary (6-17) and quaternary (21) complexes constitute a ruthenium(II) metal center whereas crystals of $\mathbf{1 8 - 2 0}$ have ruthenium(III) centers. The organization of the remaining structures, namely $\mathbf{2 2 - 7 2}$, is problematic. The common feature of $\mathbf{2 2 - 4 9}$ is the presence of at least one carbonyl ligand, 50-55, a cyclopentadienyl (Cp) ligand and 56-66, a pentamethylcyclopentadienyl $\left(\mathrm{Cp}^{*}\right)$ ligand. It is acknowledged this categorization is arbitrary. For example, the complex in crystal 55 contains both $\mathrm{CO}$ and $\mathrm{Cp}$ while crystal 66 contains both $\mathrm{CO}$ and $\mathrm{Cp}^{*}$. The last set of organometallic complexes 67-72, containing neither $\mathrm{CO}, \mathrm{Cp}$ nor $\mathrm{Cp}^{*}$, are discussed in more detail below.

Table 2. Summary of ruthenium dithiocarbamate structures established by X-ray crystallography. See Table 1, Figure 2, and the Abbreviations section.

\begin{tabular}{|c|c|c|c|c|}
\hline Crystal & Formulation & REFCODE & Donor Set & Ref. \\
\hline 1 & $\mathrm{Ru}\left(\mathrm{L}^{8}\right)_{3}$ & OCOVUZ & $\mathrm{S}_{6}$ & [25] \\
\hline 2 & $\mathrm{Ru}\left(\mathrm{L}^{20}\right)_{3}$ & OCOWEK & $\mathrm{S}_{6}$ & [25] \\
\hline 3 & {$\left[\mathrm{Ru}\left(\mathbf{L}^{57}\right)_{3}\right], \mathrm{CHCl}_{3}$} & OCOWAG & $\mathrm{S}_{6}$ & [25] \\
\hline 4 & {$\left[\mathrm{Ru}_{2}\left(\mathbf{L}^{8}\right)_{5}\right] \mathrm{BF}_{4}, \mathrm{CHCl}_{3}, \mathrm{CH}_{3} \mathrm{CN}$} & REDCEK & $\mathrm{S}_{6}$ & [32] \\
\hline 5 & {$\left[\mathrm{Ru}_{2}\left(\mathbf{L}^{8}\right)_{5}\right]\left\{\left[\mathrm{Mo}_{6} \mathrm{O}_{19}\right] 0.5\right\}, \mathrm{CH}_{3} \mathrm{COCH}_{3}$} & YUJFOZ & $\mathrm{S}_{6}$ & [33] \\
\hline 6 & $\mathrm{Ru}\left(\mathrm{L}^{8}\right)_{2}\left(\left(\mathrm{CH}_{3}\right)_{2} \mathrm{SO}\right)_{2}$ & REDBUZ & cis- $\mathrm{O}_{2} \mathrm{~S}_{4}$ & [32] \\
\hline 7 & $\mathrm{Ru}\left(\mathrm{L}^{57}\right)_{2}\left(\left(\mathrm{CH}_{3}\right)_{2} \mathrm{SO}\right)_{2}$ & REDCAG & cis- $-\mathrm{O}_{2} \mathrm{~S}_{4}$ & [32] \\
\hline 8 & $(\mathrm{dppb}) \mathrm{Ru}\left(\mathrm{L}^{24}\right)_{2}$ & EBESOW & cis- $-\mathrm{P}_{2} \mathrm{~S}_{4}$ & [34] \\
\hline 9 & {$\left[(\mathrm{dppb}) \mathrm{Ru}\left(\mathrm{L}^{63}\right)_{2}\right], \mathrm{CH}_{2} \mathrm{Cl}_{2}$} & PAZWOD & cis- $\mathrm{P}_{2} \mathrm{~S}_{4}$ & [35] \\
\hline 10 & $(\mathrm{dppb}) \mathrm{Ru}\left(\mathrm{L}^{79}\right)_{2}$ & EBESIQ & cis- $\mathrm{P}_{2} \mathrm{~S}_{4}$ & [34] \\
\hline 11 & {$\left[\mathrm{Ru}(\mathrm{bpy})_{2}\left(\mathrm{~L}^{8}\right)\right] \mathrm{PF}_{6}, 0.75\left(\left(\mathrm{CH}_{3} \mathrm{CH}_{2}\right)_{2} \mathrm{O}\right)$} & DANDOM & $\mathrm{N}_{4} \mathrm{~S}_{2}$ & [36] \\
\hline 12 & $\left.\left[\mathrm{Ru}(\mathrm{bpy})_{2} \mathbf{L}^{56}\right)\right] \mathrm{PF}_{6}$ & WOHLAH & $\mathrm{N}_{4} \mathrm{~S}_{2}$ & [37] \\
\hline 13 & {$\left[\mathrm{Ru}(\right.$ cyclam $\left.)\left(\mathrm{L}^{24}\right)\right] \mathrm{BPh}_{4}, 0.16\left(\mathrm{CH}_{2} \mathrm{Cl}_{2}\right)$} & BUPMEF & $\mathrm{N}_{4} \mathrm{~S}_{2}$ & [38] \\
\hline 14 & {$\left[(\mathrm{dppm})_{2} \mathrm{Ru}\left(\mathrm{L}^{9}\right)\right] \mathrm{PF}_{6}, 0.5\left(\mathrm{CH}_{2} \mathrm{Cl}_{2}\right)$} & COZMIP & $\mathrm{P}_{4} \mathrm{~S}_{2}$ & [39] \\
\hline 15 & {$\left[(\mathrm{dppm})_{2} \mathrm{Ru}\left(\mathrm{L}^{26}\right)\right] \mathrm{PF}_{6}$} & HUYPAT & $\mathrm{P}_{4} \mathrm{~S}_{2}$ & [40] \\
\hline 16 & $\left\{\left[(\mathrm{dppm})_{2} \mathrm{Ru}\right]_{2}\left(\mathrm{~L}^{74}\right)\right\}\left[\mathrm{BF}_{4}\right]_{2}, 3\left(\mathrm{CHCl}_{3}\right)$ & QAVHAX & $\mathrm{P}_{4} \mathrm{~S}_{2}$ & [41] \\
\hline 17 & {$\left[(\mathrm{dppm})_{2} \mathrm{Ru}\left(\mathrm{L}^{74}\right) \mathrm{Pd}\left(\mathrm{PPh}_{3}\right)_{2}\right]\left[\mathrm{BF}_{4}\right]_{2}, 3\left(\mathrm{CH}_{2} \mathrm{Cl}_{2}\right),\left(\mathrm{CH}_{3} \mathrm{CH}_{2}\right)_{2} \mathrm{O}$} & QONVAR & $\mathrm{P}_{4} \mathrm{~S}_{2}$ & [42] \\
\hline 18 & $\left(\mathrm{PPh}_{3}\right)_{2} \mathrm{Ru}\left(\mathrm{L}^{8}\right) \mathrm{Cl}_{2}, 2\left(\mathrm{CH}_{2} \mathrm{Cl}_{2}\right)$ & HUPMAH & $\mathrm{Cl}_{2} \mathrm{P}_{2} \mathrm{~S}_{2}$ & [43] \\
\hline 19 & $\left(\mathrm{PPh}_{3}\right)_{2} \mathrm{Ru}\left(\mathrm{L}^{24}\right) \mathrm{Cl}_{2}, \mathrm{CH}_{2} \mathrm{Cl}_{2}$ & GUVPIX & $\mathrm{Cl}_{2} \mathrm{P}_{2} \mathrm{~S}_{2}$ & [44] \\
\hline 20 & $\left(\mathrm{PPh}_{3}\right)_{2} \mathrm{Ru}\left(\mathrm{L}^{29}\right) \mathrm{Cl}_{2}, \mathrm{CH}_{2} \mathrm{Cl}_{2}$ & GUVPOD & $\mathrm{Cl}_{2} \mathrm{P}_{2} \mathrm{~S}_{2}$ & [44] \\
\hline 21 & {$\left[\left(\mathrm{PPh}_{3}\right) \mathrm{Ru}(\mathrm{tptz})\left(\mathrm{L}^{24}\right)\right] \mathrm{Cl}, 2.5\left(\mathrm{H}_{2} \mathrm{O}\right)$} & ABIBET & $\mathrm{N}_{3} \mathrm{PS}_{2}$ & [45] \\
\hline 22 & $\left(\mathrm{PEt}_{3}\right) \mathrm{Ru}\left(\mathbf{L}^{8}\right)_{2}(\mathrm{CO})$ & PEBZUS & $\mathrm{CPS}_{4}$ & [46] \\
\hline 23 & $\left(\mathrm{PPh}_{3}\right) \mathrm{Ru}\left\{\mathrm{Ge}(\mathrm{p} \text {-tolyl })_{2}\right\}\left(\mathbf{L}^{8}\right)_{2}(\mathrm{CO}), \mathrm{CH}_{2} \mathrm{Cl}_{2}$ & VEKKIH & $\mathrm{CGePS}_{3}$ & [47] \\
\hline 24 & $\left(\mathrm{PPh}_{3}\right) \mathrm{Ru}\left(\mathrm{K}^{2}(\mathrm{Si}, \mathrm{N})-\mathrm{SiPh}_{2} \mathrm{NHC}_{5} \mathrm{H}_{4} \mathrm{~N}\right)\left(\mathbf{L}^{8}\right)(\mathrm{CO})$ & RADNIU & $\mathrm{CNPS}_{2} \mathrm{Si}$ & [48] \\
\hline 25 & $\left(\mathrm{PPh}_{3}\right) \mathrm{Ru}\left(\kappa^{2}(\mathrm{Si}, \mathrm{N})-\mathrm{SiPh}_{2} \mathrm{OC}_{5} \mathrm{H}_{4} \mathrm{~N}\right)\left(\mathrm{L}^{8}\right)(\mathrm{CO}), 2.5\left(\mathrm{C}_{6} \mathrm{H}_{6}\right)$ & RADNEQ & $\mathrm{CNPS}_{2} \mathrm{Si}$ & [48] \\
\hline 26 & $\left(\mathrm{PPh}_{3}\right) \mathrm{Ru}\left(\mathrm{k}^{2}(\mathrm{Si}, \mathrm{O})-\mathrm{SiPh} 2 \mathrm{OCCH} \mathrm{C}_{3} \mathrm{O}\right)\left(\mathrm{L}^{8}\right)(\mathrm{CO}), \mathrm{CH}_{2} \mathrm{Cl}_{2}$ & RADNOA & $\mathrm{CNPS}_{2} \mathrm{Si}$ & [48] \\
\hline 27 & $\mathrm{Ru}\left(\mathrm{B}\left(\mathrm{NCH}_{2} \mathrm{PPh}_{2}\right)_{2} \mathrm{C}_{6} \mathrm{H}_{4}\right)\left(\mathrm{L}^{24}\right)(\mathrm{CO})$ & UNAVEM & $\mathrm{BCP}_{2} \mathrm{~S}_{2}$ & [49] \\
\hline 28 & $\left(\mathrm{PPh}_{3}\right)_{2} \mathrm{Ru}(\mathrm{H})\left(\mathrm{L}^{7}\right)(\mathrm{CO}), 0.5\left(\mathrm{CH}_{3}\left(\mathrm{CH}_{2}\right)_{4} \mathrm{CH}_{3}\right)$ & OWOKER & $\mathrm{CHP}_{2} \mathrm{~S}_{2}$ & [50] \\
\hline 29 & $\left(\mathrm{PPh}_{3}\right)_{2} \mathrm{Ru}(\mathrm{H})\left(\mathbf{L}^{8}\right)(\mathrm{CO})$ & RADMUF & $\mathrm{CHP}_{2} \mathrm{~S}_{2}$ & [48] \\
\hline 30 & $\left(\mathrm{PPh}_{3}\right)_{2} \mathrm{Ru}(\mathrm{H})\left(\mathrm{L}^{84}\right)(\mathrm{CO})$ & YOSJEX & $\mathrm{CHP}_{2} \mathrm{~S}_{2}$ & [51] \\
\hline 31 & $\left(\mathrm{PPh}_{3}\right)_{2} \mathrm{Ru}\left(\mathrm{Si}(\mathrm{Cl}) \mathrm{Ph}_{2}\right)\left(\mathrm{L}^{8}\right)(\mathrm{CO})$ & RADNAM & $\mathrm{CP}_{2} \mathrm{~S}_{2} \mathrm{Si}$ & [48] \\
\hline 32 & $\left(\mathrm{PPh}_{3}\right)_{2} \mathrm{Ru}\left(\mathrm{Ge}(\mathrm{p}-\text { tolyl })_{3}\right)\left(\mathrm{L}^{24}\right)(\mathrm{CO}), 1.19\left(\mathrm{CH}_{2} \mathrm{Cl}_{2}\right)$ & VEKKAZ & $\mathrm{CGeP}_{2} \mathrm{~S}_{2}$ & [47] \\
\hline 33 & $\left(\mathrm{PPh}_{3}\right)_{2} \mathrm{Ru}(\mathrm{C}(\mathrm{H})=\mathrm{C}(\mathrm{H}) \mathrm{BMIDA})\left(\mathrm{L}^{24}\right)(\mathrm{CO}), \mathrm{CH}_{3} \mathrm{COCH}_{3}$ & HUDPON & $\mathrm{C}_{2} \mathrm{P}_{2} \mathrm{~S}_{2}$ & [52] \\
\hline 34 & $\left(\mathrm{PPh}_{3}\right)_{2} \mathrm{Ru}\left(\mathrm{C}(\mathrm{H})=\mathrm{C}(\mathrm{H}) \mathrm{C}_{6} \mathrm{H}_{4} \mathrm{Me}-4\right)\left(\mathrm{L}^{10}\right)(\mathrm{CO}), \mathrm{CH}_{2} \mathrm{Cl}_{2}$ & COZMEL & $\mathrm{C}_{2} \mathrm{P}_{2} \mathrm{~S}_{2}$ & [39] \\
\hline 35 & $\left(\mathrm{PPh}_{3}\right)_{2} \mathrm{Ru}\left(\mathrm{C}(\mathrm{H})=\mathrm{C}(\mathrm{H}) \mathrm{C}_{6} \mathrm{H}_{4} \mathrm{Me}-4\right)\left(\mathrm{L}^{26}\right)(\mathrm{CO}), 0.3\left(\mathrm{CH}_{2} \mathrm{Cl}_{2}\right)$ & HUYPEX & $\mathrm{C}_{2} \mathrm{P}_{2} \mathrm{~S}_{2}$ & [40] \\
\hline 36 & $\left(\mathrm{PPh}_{3}\right)_{2} \mathrm{Ru}\left(\mathrm{C}(\mathrm{H})=\mathrm{C}(\mathrm{H}) \mathrm{C}_{6} \mathrm{H}_{4} \mathrm{Me}-4\right)\left(\mathrm{L}^{27}\right)(\mathrm{CO}), \mathrm{CH}_{2} \mathrm{Cl}_{2}$ & OJEYIM & $\mathrm{C}_{2} \mathrm{P}_{2} \mathrm{~S}_{2}$ & [53] \\
\hline 37 & $\left(\mathrm{PPh}_{3}\right)_{2} \mathrm{Ru}\left(\mathrm{C}(\mathrm{H})=\mathrm{C}(\mathrm{H})\right.$ pyrenyl-1) $\left(\mathrm{L}^{84}\right)(\mathrm{CO}), 1.5\left(\mathrm{CH}_{2} \mathrm{Cl}_{2}\right)$ & YOSJIB & $\mathrm{C}_{2} \mathrm{P}_{2} \mathrm{~S}_{2}$ & [51] \\
\hline 38 & $\left(\mathrm{PPh}_{3}\right)_{2} \mathrm{Ru}\left(\mathrm{C}(\mathrm{C} \equiv \mathrm{C}(\mathrm{Ph})=\mathrm{C}(\mathrm{H}) \mathrm{Ph})\left(\mathrm{L}^{84}\right)(\mathrm{CO})\right.$ & YOSJOH & $\mathrm{C}_{2} \mathrm{P}_{2} \mathrm{~S}_{2}$ & [51] \\
\hline
\end{tabular}


Table 2. Cont.

\begin{tabular}{|c|c|c|c|c|}
\hline Crystal & Formulation & REFCODE & Donor Set & Ref. \\
\hline 39 & $\left(\mathrm{PPh}_{3}\right)_{2} \mathrm{Ru}\left(\mathrm{C}\left(\mathrm{C} \equiv \mathrm{CSiMe}_{3}\right)=\mathrm{C}(\mathrm{H}) \mathrm{SiMe}_{3}\right)\left(\mathrm{L}^{8}\right)(\mathrm{CO})$, unknown solvate & KOQPEO & $\mathrm{C}_{2} \mathrm{P}_{2} \mathrm{~S}_{2}$ & [54] \\
\hline 40 & {$\left[\left(\mathrm{PPh}_{3}\right)_{2} \mathrm{Ru}(\mathrm{C}(\mathrm{C} \equiv \mathrm{CPh})=\mathrm{C}(\mathrm{H}) \mathrm{Ph})\left(\mathrm{L}^{65}\right)(\mathrm{CO})\right] \mathrm{Cl}, 1.25\left(\mathrm{CH}_{2} \mathrm{CH}_{2} \mathrm{OH}\right)$} & DOZHOQ & $\mathrm{C}_{2} \mathrm{P}_{2} \mathrm{~S}_{2}$ & [55] \\
\hline 41 & $\left(\mathrm{PPh}_{3}\right)_{2} \mathrm{Ru}(\mathrm{C} \equiv \mathrm{CBMIDA})\left(\mathrm{L}^{8}\right)(\mathrm{CO})$ & HUDPAZ & $\mathrm{C}_{2} \mathrm{P}_{2} \mathrm{~S}_{2}$ & [52] \\
\hline 42 & $\left(\mathrm{PPh}_{3}\right)_{2} \mathrm{Ru}(\mathrm{C} \equiv \mathrm{CBMIDA})\left(\mathrm{L}^{24}\right)(\mathrm{CO}), 0.5\left(\mathrm{CH}_{2} \mathrm{Cl}_{2}\right)$, unknown solvate & HUDPED & $\mathrm{C}_{2} \mathrm{P}_{2} \mathrm{~S}_{2}$ & [52] \\
\hline 43 & $\left(\mathrm{PPh}_{3}\right)_{2} \mathrm{Ru}(\mathrm{X})\left(\mathrm{L}^{24}\right)(\mathrm{CO})$ & ВOHТOK & $\mathrm{C}_{2} \mathrm{P}_{2} \mathrm{~S}_{2}$ & [56] \\
\hline 44 & $\left(\mathrm{PPh}_{3}\right)_{2} \mathrm{Ru}\left(\mathrm{L}^{24}\right)(\mathrm{CO}) \mathrm{Cl}, 2.5\left(\mathrm{C}_{6} \mathrm{H}_{6}\right)$ & HUDQAA & $\mathrm{CClP}_{2} \mathrm{~S}_{2}$ & [52] \\
\hline 45 & $\left(\mathrm{PPh}_{3}\right)_{2} \mathrm{Ru}\left(\mathrm{L}^{24}\right)(\mathrm{CO}) \mathrm{Cl}, \mathrm{CH}_{2} \mathrm{Cl}_{2}$ & HUDQEE & $\mathrm{CClP}_{2} \mathrm{~S}_{2}$ & [52] \\
\hline 46 & $\left(\mathrm{PPh}_{3}\right)_{2} \mathrm{Ru}\left(\mathrm{L}^{24}\right)(\mathrm{CO}) \mathrm{Cl}, 2\left(\mathrm{CHCl}_{3}\right)$, unknown solvate & HUDQII & $\mathrm{CClP}_{2} \mathrm{~S}_{2}$ & [52] \\
\hline 47 & $\left(\mathrm{PPh}_{3}\right)_{2} \mathrm{Ru}\left(\mathrm{L}^{24}\right)(\mathrm{CO}) \mathrm{Cl}, \mathrm{CH}_{3} \mathrm{OH}$ & HUDQOO & $\mathrm{CClP}_{2} \mathrm{~S}_{2}$ & [52] \\
\hline 48 & $\left(\mathrm{PPh}_{3}\right) \mathrm{Ru}\left(\mathrm{Ge}(\mathrm{p} \text {-tolyl })_{3}\right)\left(\mathrm{L}^{24}\right)(\mathrm{CO})_{2}$ & VEKKED & $\mathrm{C}_{2} \mathrm{GePS}_{2}$ & [47] \\
\hline 49 & $\mathrm{Ru}\left(\mathrm{L}^{22}\right)(\mathrm{CO})_{3} \mathrm{Cl}$ & ROQQUM & fac- $\mathrm{C}_{3} \mathrm{ClS}_{2}$ & [57] \\
\hline 50 & $\mathrm{CpRu}\left(\mathrm{PPh}_{3}\right)\left(\mathbf{L}^{\mathbf{1 1}}\right)$ & YEDMEA & $\mathrm{C}_{3} \mathrm{PS}_{2}$ & [58] \\
\hline 51 & $\mathrm{CpRu}\left(\mathrm{PPh}_{3}\right)\left(\mathrm{L}^{24}\right)$ & KEZVOB & $\mathrm{C}_{3} \mathrm{PS}_{2}$ & [59] \\
\hline 52 & $\mathrm{CpRu}\left(\mathrm{PPh}_{3}\right)\left(\mathbf{L}^{28}\right)$ & YEDMAW & $\mathrm{C}_{3} \mathrm{PS}_{2}$ & [58] \\
\hline 53 & $\mathrm{CpRu}\left(\mathrm{PPh}_{3}\right)\left(\mathrm{L}^{57}\right)$ & BUYQIW & $\mathrm{C}_{3} \mathrm{PS}_{2}$ & [60] \\
\hline 54 & $\left\{\left[\mathrm{CpRu}\left(\mathrm{L}^{24}\right)\right]_{2}(\mathrm{dppf})\right\}, \mathrm{CH}_{2} \mathrm{Cl}_{2}$ & IZIBAU & $\mathrm{C}_{3} \mathrm{PS}_{2}$ & [61] \\
\hline 55 & $\mathrm{CpRu}\left(\mathrm{L}^{8}\right)(\mathrm{CO})$ & QIWNES & $\mathrm{C}_{4} \mathrm{~S}_{2}$ & [62] \\
\hline 56 & {$\left[\mathrm{Cp} * \mathrm{Ru}\left(\mathrm{L}^{8}\right)_{2}\right]\left[\mathrm{N}\left(\mathrm{S}=\mathrm{PPh}_{2}\right)_{2}\right], 0.5\left(\mathrm{H}_{2} \mathrm{O}\right)$} & PARVUA & $\mathrm{C}_{3} \mathrm{~S}_{4}$ & [63] \\
\hline 57 & {$\left[\mathrm{Cp} * \mathrm{Ru}\left(\mathrm{L}^{8}\right)\left(\mathrm{L}^{24}\right)\right] \mathrm{Cl}, 2 \mathrm{CHCl}_{3}$} & TEYTEX & $\mathrm{C}_{3} \mathrm{~S}_{4}$ & [64] \\
\hline 58 & $\mathrm{Cp} \mathrm{p}^{*} \mathrm{Ru}\left(\mathrm{L}^{8}\right) \mathrm{Cl}_{2}$ & WEYTUQ & $\mathrm{C}_{3} \mathrm{Cl}_{2} \mathrm{~S}_{2}$ & [65] \\
\hline 59 & $\mathrm{Cp} \mathrm{p}^{*} \mathrm{Ru}\left(\mathrm{L}^{24}\right) \mathrm{Cl}_{2}$ & TEYSUM & $\mathrm{C}_{3} \mathrm{Cl}_{2} \mathrm{~S}_{2}$ & [64] \\
\hline 60 & $\mathrm{Cp}{ }^{*} \mathrm{Ru}\left(\mathrm{S}_{2} \mathrm{C}=\mathrm{O}\right)\left(\mathrm{L}^{8}\right)$ & TEYTIB & $\mathrm{C}_{3} \mathrm{~S}_{4}$ & [64] \\
\hline 61 & $\mathrm{Cp} \mathrm{p}^{*} \mathrm{Ru}\left(\mathrm{S}_{2} \mathrm{C}=\mathrm{O}\right)\left(\mathrm{L}^{24}\right)$ & TEYTOH & $\mathrm{C}_{3} \mathrm{~S}_{4}$ & [64] \\
\hline 62 & {$\left[\mathrm{Cp} * \mathrm{Ru}\left(\mathrm{PPh}_{3}\right)\left(\mathrm{L}^{8}\right) \mathrm{Cl}\right] \mathrm{Cl}, 0.5\left(\mathrm{H}_{2} \mathrm{O}\right), 1.5\left(\mathrm{CH}_{3} \mathrm{CN}\right)$} & TEYTUN & $\mathrm{C}_{3} \mathrm{ClPS}_{2}$ & [64] \\
\hline 63 & {$\left[\mathrm{Cp} * \mathrm{Ru}\left(\mathrm{PMe}_{3}\right)\left(\mathrm{L}^{8}\right) \mathrm{Cl}\right] \mathrm{BPh}_{4}$} & TEYVAV & $\mathrm{C}_{3} \mathrm{ClPS}_{2}$ & [64] \\
\hline 64 & {$\left[\mathrm{Cp} * \mathrm{Ru}\left(\mathrm{L}^{8}\right) \mathrm{Cl}\right]_{2}\left[\mathrm{SbCl}_{6}\right]_{2}$} & WEYVEC & $\mathrm{C}_{3} \mathrm{Cl}_{2} \mathrm{~S}_{2}$ & [65] \\
\hline 65 & {$\left[\mathrm{Cp} * \mathrm{Ru}\left(\mathrm{L}^{8}\right)\left(\mathrm{CH}_{3} \mathrm{CN}\right)_{2}\right]\left[\mathrm{PF}_{6}\right]_{2}, 2 \mathrm{CH}_{3} \mathrm{CN}$} & WEYVAY & $\mathrm{C}_{3} \mathrm{~N}_{2} \mathrm{~S}_{2}$ & [65] \\
\hline 66 & $\mathrm{Cp}^{*} \mathrm{Ru}\left(\mathrm{L}^{8}\right)(\mathrm{CO})$ & TEYVID & $\mathrm{C}_{4} \mathrm{~S}_{2}$ & [64] \\
\hline 67 & {$\left[\left(\mathrm{PPh}_{3}\right)_{2} \mathrm{Ru}\left(\mathrm{C}\left(\mathrm{NMe}_{2}\right) \mathrm{OC}\left(\mathrm{NMe}_{2}\right)=\mathrm{S}\right)\left(\mathrm{L}^{8}\right)\right] \mathrm{ClO}_{4}$} & HUPMEL & $\mathrm{CP}_{2} \mathrm{~S}_{3}$ & [43] \\
\hline 68 & {$\left[\left(\mathrm{PPh}_{3}\right)_{2} \mathrm{Ru}\left(\mathrm{C}\left(\mathrm{NMe}_{2}\right) \mathrm{SC}\left(\mathrm{NMe}_{2}\right)=\mathrm{S}\right)\left(\mathrm{L}^{8}\right)\right] \mathrm{ClO}_{4}, 2\left(\mathrm{CH}_{2} \mathrm{Cl}_{2}\right)$} & HUPLUA & $\mathrm{CP}_{2} \mathrm{~S}_{3}$ & [43] \\
\hline 69 & $\mathrm{Ru}\left(X^{1}\right)\left(\mathbf{L}^{8}\right) \mathrm{Cl}$ & IQUFEG & $\mathrm{C}_{4} \mathrm{ClS}_{2}$ & [66] \\
\hline 70 & $\mathrm{Ru}\left(\mathrm{X}^{1}\right)\left(\mathrm{L}^{29}\right) \mathrm{Cl}$ & IQUFIK & $\mathrm{C}_{4} \mathrm{ClS}_{2}$ & [66] \\
\hline 71 & {$\left[\mathrm{Ru}\left(\mathrm{X}^{1}\right)\left(\mathbf{L}^{8}\right)\left(\mathrm{CH}_{3} \mathrm{CN}\right)\right] \mathrm{PF}_{6}$} & IQUFOQ & $\mathrm{C}_{4} \mathrm{NS}_{2}$ & [66] \\
\hline 72 & $(\mathrm{p}$-cymene $) \mathrm{Ru}\left(\mathrm{L}^{79}\right) \mathrm{Cl}$ & PEKVIN & $\mathrm{C}_{3} \mathrm{ClS}_{2}$ & [67] \\
\hline
\end{tabular}

Crystals 1-3 [25] feature binary tris dithiocarbamate complexes. The presence of three symmetrically chelating ligands defines an octahedral geometry and enables the formation of enantiomers, i.e., $\Delta$ and $\Lambda$. A representative example is shown in Figure 3a being the $\Delta$-enantiomer of $\mathbf{1}$. The Ru-S bond lengths are equivalent and the main distortions from the ideal geometry arise as a consequence of the small bite angle of the chelating dithiocarbamate ligands. Two crystals have dinuclear $\left[\mathrm{Ru}_{2}\left(\mathrm{~L}^{8}\right)_{5}\right]^{+}$cations. Despite the common formulation, two distinct patterns of coordination are noted. In the crystal of 4 [32], Figure 3b, two dithiocarbamate ligands bridge the two ruthenium atoms, chelating one and simultaneously bridging the other via one sulfur atom only. The third ligand is also bridging but forms only one bond to each ruthenium atom; the remaining dithiocarbamate ligands are chelating. As shown in Figure 3c, in 5 [33], two dithiocarbamate ligands bridge the two ruthenium atoms by chelating one and simultaneously bridging the other via one sulfur atom only. The bridging dithiocarbamate ligands are connected to the same ruthenium atom. The $\mathrm{S}_{6}$-octahedral geometry for each metal center is completed by one and two chelating ligands, respectively. The presence of different counter ions and solvates in the crystal may be responsible for the observed behavior. 


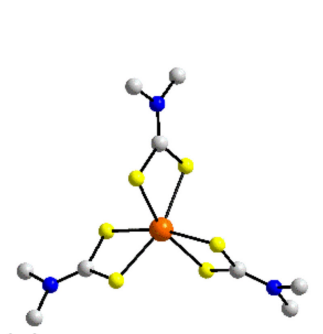

(a)

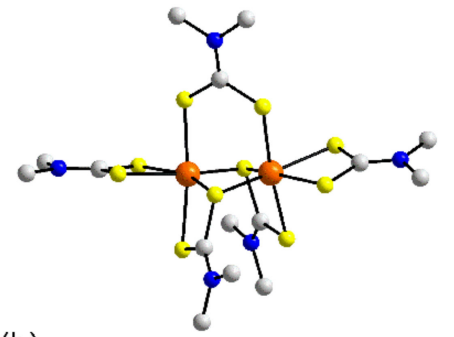

(b)

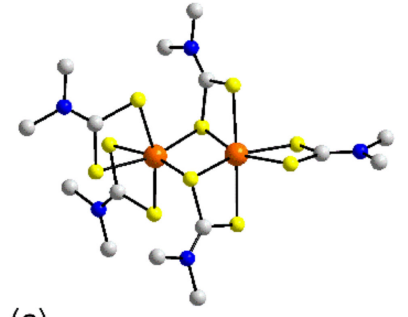

(c)

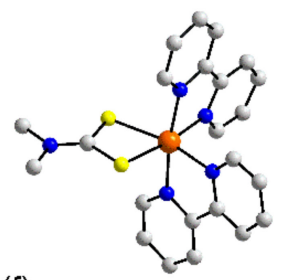

(f)

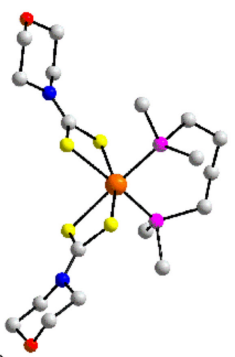

(e)

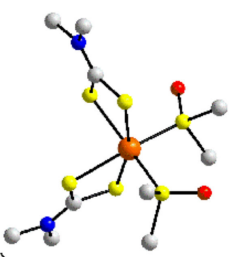

(d)

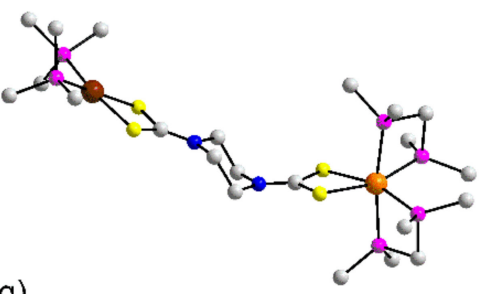

(g)

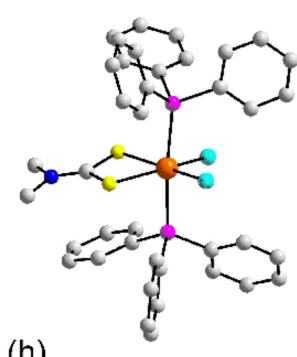

(h)

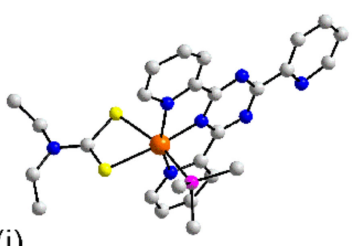

(i)

Figure 3. Molecular structures of ruthenium complexes: $(\mathbf{a}) \operatorname{Ru}\left(\mathbf{L}^{8}\right)_{3}(\mathbf{1}),(\mathbf{b})\left[R u\left(\mathbf{L}^{8}\right)_{3}\right]$ in the crystal of 4, (c) $\left[\mathrm{Ru}_{2}\left(\mathbf{L}^{8}\right)_{3}\right]$ in 5, (d) $\mathrm{Ru}\left(\mathbf{L}^{8}\right)_{2}\left(\left(\mathrm{CH}_{3}\right)_{2} \mathrm{SO}\right)_{2}(\mathbf{6}),(\mathbf{e})(\mathrm{dppb}) \mathrm{Ru}\left(\mathrm{L}^{79}\right)_{2}(\mathbf{1 0}),(\mathbf{f})\left[\mathrm{Ru}(\mathrm{bpy})_{2}\left(\mathbf{L}^{8}\right)\right]^{+}$in 11, (g) $\left[(\mathrm{dppm})_{2} \mathrm{Ru}\left(\mathrm{L}^{74}\right) \mathrm{Pd}\left(\mathrm{PPh}_{3}\right)_{2}\right]^{2+}$ in $\mathbf{1 7}$, (h) $\left[\left(\mathrm{PPh}_{3}\right)_{2} \mathrm{Ru}\left(\mathbf{L}^{8}\right) \mathrm{Cl}_{2}\right]$ in $\mathbf{1 8}$, and (i) $\left[\left(\mathrm{PPh}_{3}\right) \mathrm{Ru}(\mathrm{tptz})\left(\mathrm{L}^{24}\right)\right]^{+}$ in 21. In $(\mathbf{e}, \mathbf{g}, \mathbf{i})$, only the ispo-C atoms of the phosphane bound phenyl rings are shown for clarity. Color code in this and subsequent crystallographic images: platinum-group element, orange; other heavy element, brown; chloride, cyan; sulfur, yellow; phosphorus, purple; oxygen, red; nitrogen, blue; carbon, grey; hydrogen, green. Non-participating $\mathrm{H}$ atoms are omitted.

Attention now turns to ternary complexes. In common with 1-5, mononuclear complexes in 6 [32], Figure 3d, and 7 [32] feature octahedral geometries defined by $\mathrm{S}_{6}$ donor sets. In the latter, the S-bound DMSO molecules occupy cis-positions with the dithiocarbamate ligands chelating in a slightly asymmetric mode with the longer Ru-S(dithiocarbamate) bonds occupying positions trans to the DMSO-S atoms. In crystals, 8 [34], 9 [35], and 10 [34], Figure 3e, the DMSO molecules of 6 and 7 have been substituted by a chelating dppb ligand leading to cis- $\mathrm{P}_{2} \mathrm{~S}_{4}$ donor sets. The number of dithiocarbamate ligands in the remaining structures, $\mathbf{1 1}$ to $\mathbf{2 1}$ is reduced to one. In the cationic salts $\mathbf{1 1}$ [36], Figure 3f, and 12 [37], the 2,2'-bipyridyl ligands are each chelating so the donor set is cis- $\mathrm{N}_{4} \mathrm{~S}_{2}$. A similar geometry is found for the cation in 13 [38] where the four nitrogen atoms of the former examples are now replaced by the amine-nitrogen atoms of the cyclam ligand. The ruthenium center in the complexes of crystals $\mathbf{1 4}$ [39], 15 [40], 16 [41], and 17 [42] have the common feature of bearing two chelating dppm ligands and in a sense, the resulting cis- $\mathrm{P}_{4} \mathrm{~S}_{2}$ coordination geometries are the inverse to those seen in the crystals of 8 to 10 . The heterometallic dinuclear complex in 17, Figure $3 \mathrm{~g}$, is worthy of special mention in that the ruthenium and square planar palladium atoms are connected by a multifunctional dithiocarbamate dianion, $\mathbf{L}^{74}$. The neutral complexes in quaternary crystals of $\mathbf{1 8}$ [43], 19 [44], and 20 [44] have trans $\mathrm{PPh}_{3}$ ligands with the chloride atoms occupying positions trans to 
the sulfur atoms of the chelating dithiocarbamate ligands; a representative molecule, i.e., 18 is illustrated in Figure $3 \mathrm{~h}$. The final non-organometallic molecule to be described in this section is the cation in 21 [45]. Here, the nitrogen atoms of the tridentate tptz ligand occupy mer-positions in the octahedral geometry defined by the $\mathrm{N}_{3} \mathrm{PS}_{2}$ donor set, Figure $3 \mathrm{i}$.

The common feature of organometallic species $22-49$ is the presence of at least one carbonyl ligand: indeed, all but 48 (two) and 49 (three) have one terminally bound carbonyl group. In each of 22 [46] and 23 [47], there are two dithiocarbamate ligands while the remaining structures have one dithiocarbamate each. In 22, Figure 4a, the ruthenium(II) center is bis chelated by two dithiocarbamate ligands with the distorted octahedral geometry completed by the phosphane and carbonyl ligands. In 23, Figure 4b, one of the dithiocarbamate ligands of $\mathbf{2 2}$ now bridges an already coordinated germanium atom, forming one bond to each atom with the resultant octahedral coordination geometry based on a $\mathrm{CGePS}_{3}$ donor set. The common feature of 24-26 [48] is a $\mathrm{CNPS}_{2} \mathrm{Si}$ donor set as one of the chelating dithiocarbamate ligands of 22 is replaced by a $\mathrm{Si} \cap \mathrm{N}$ chelating ligand with the difference in structures related to the backbone of the $\mathrm{Si} \cap \mathrm{N}$ chelating ligand; the molecule in 24 is shown in Figure 4c. In 27 [49], the dithiocarbamate and phosphane ligands of 22 are replaced by a tridentate ligand where coordination occurs from the ring-boron atom and two pendant phosphane ligands, Figure $4 \mathrm{~d}$. Thus, this is the first example where ruthenium is coordinated by two phosphane ligands representing a key structural difference from 22-26. The common feature of the complexes in the crystals of 28-47 is that they conform to the general formula $\left(\mathrm{PPh}_{3}\right)_{2} \mathrm{Ru}\left(\mathbf{L}^{\mathbf{n}}\right)(\mathrm{CO}) \mathrm{Y}$. For 28 [50], 29 [48], Figure 4e, and 30 [51], $\mathrm{Y}$ is a hydride. In common with all structures up to an including 43 , the phosphane ligands are mutually trans. Similar arrangements are found in 31 [48] and 32 [47] where the hydride has been substituted for $\mathrm{Si}(\mathrm{Cl}) \mathrm{Ph}_{2}$ and $\mathrm{Ge}(\mathrm{p} \text {-tolyl) })_{3}$ groups, respectively.

The $\left(\mathrm{PPh}_{3}\right)_{2} \mathrm{Ru}\left(\mathbf{L}^{\mathbf{n}}\right)(\mathrm{CO})$ framework figures prominently in the stabilization of a variety of ruthenium-bound alkenes and alkynes. Alkenes of the type $\mathrm{C}(\mathrm{H})=\mathrm{C}(\mathrm{H}) \mathrm{R}$ feature in the structures of 33 [52], Figure 4f, 34 [39], 35 [40], 36 [53], and 37 [51], whereas those of the type $\left.C\left(C \equiv R^{\prime}\right)\right)=C(H) R$ are seen in 38 [51], Figure $4 \mathrm{~g}, 39$ [54], and salt 40 [55]. Alkynes are observed in 41 [52] and 42 [52]. In 43 [56], Figure 4h, there is a Ru-C(phenyl) bond. The introduction of chloride to replace the alkene, alkyne, and phenyl ligands of 33-43 results in a major readjustment of the coordination geometry in that the phosphane ligands are now cis. The crystals of 44-47 [52] contain the same complex species but differ in the nature of the crystalline solvent; the molecule of 44 , being representative, is shown in Figure $4 \mathrm{i}$. The complex molecule in 48 [47] is different from the previous carbonyl compounds in that there are two carbonyl groups, occupying positions trans to the dithiocarbamate-sulfur atoms; the phosphane and $\mathrm{Ge}(\mathrm{p} \text {-tolyl })_{3}$ ligands are trans within the octahedral geometry defined by a $\mathrm{C}_{2} \mathrm{GePS}_{2}$ donor set, Figure $4 \mathrm{j}$. In 49 [57], a tri-carbonyl species lacking a phosphane ligand, the carbonyls occupy one octahedral face with the remaining sites defined by dithiocarbamate-sulfur and chloride atoms, Figure $4 \mathrm{k}$.

The other common ligands featuring in the ruthenium dithiocarbamates are those based on cyclopentadienyl, namely the parent $\mathrm{Cp}$ and permethylated $\mathrm{Cp} \mathrm{p}^{*}$ ligands. Molecules in the crystals 50-55 feature $\mathrm{Cp}$ and three district structural motifs are evident. The molecules in 50 [58], 51 [59], 52 [58], and 53 [60] conform to the same motif shown in Figure 5 a for 50 , notable for having a dissymmetric dithiocarbamate ligand. The ruthenium is coordinated by a chelating dithiocarbamate ligand, a phosphane and the three remaining positions in the octahedral geometry defined by the $\mathrm{Cp}$ ligand. A similar coordination geometry pertains in the dinuclear species 54 [61] where two ruthenium atoms are linked by a dppf molecule, Figure 5b. In 55 [62], a mixed carbonyl/cyclopentadienyl compound, the former effectively substitutes for a phosphane ligand leading to an octahedral geometry-based, formally, on a $\mathrm{C}_{4} \mathrm{~S}_{2}$ donor set, Figure $5 \mathrm{c}$. 


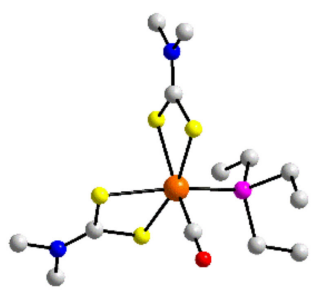

(a)

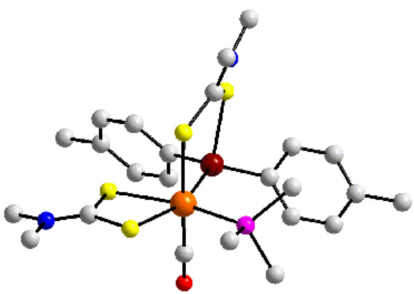

(b)

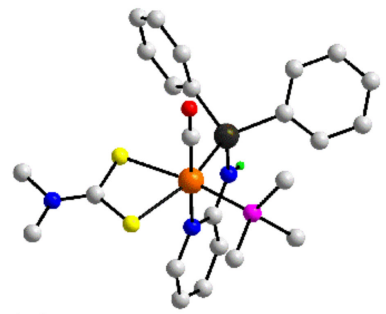

(c)

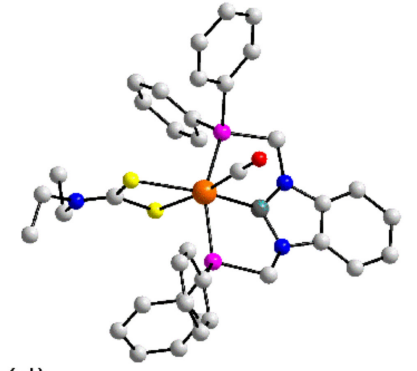

(d)

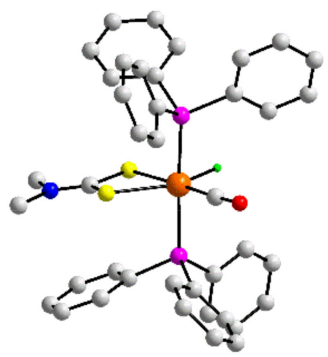

(e)

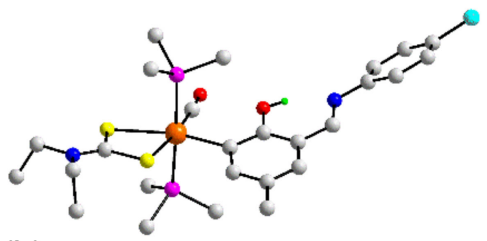

(h)

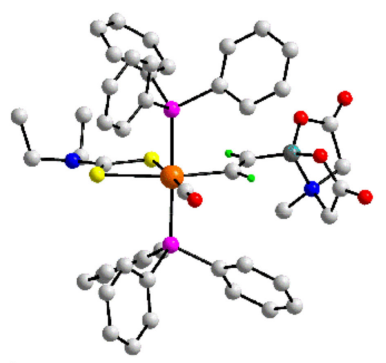

(f)

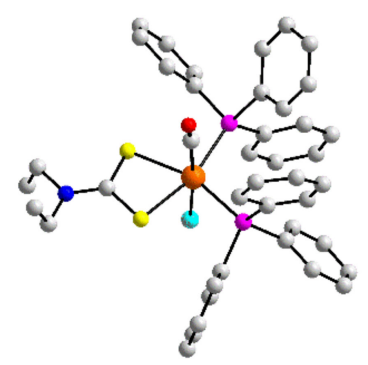

(i)

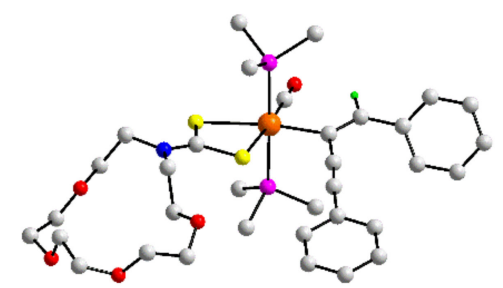

(g)

Figure 4. Molecular structures of ruthenium complexes: (a) $\left(\mathrm{PEt}_{3}\right) \mathrm{Ru}\left(\mathrm{L}^{8}\right)_{2}(\mathrm{CO})(\mathbf{2 2}),(\mathbf{b})\left(\mathrm{PPh}_{3}\right) \mathrm{Ru}\left\{\mathrm{Ge}(\mathrm{p} \text {-tolyl })_{2}\right\}\left(\mathrm{L}^{8}\right)_{2}(\mathrm{CO})$ in the crystal of 23, (c) $\left(\mathrm{PPh}_{3}\right) \mathrm{Ru}\left(\kappa^{2}(\mathrm{Si}, \mathrm{N})-\mathrm{SiPh}_{2} \mathrm{NHC}_{5} \mathrm{H}_{4} \mathrm{~N}\right)\left(\mathrm{L}^{8}\right)(\mathrm{CO})(\mathbf{2 4})$, (d) $\mathrm{Ru}\left(\mathrm{B}\left(\mathrm{NCH}_{2} \mathrm{PPh}_{2}\right)_{2} \mathrm{C}_{6} \mathrm{H}_{4}\right)\left(\mathrm{L}^{24}\right)(\mathrm{CO})(\mathbf{2 7})$, (e) $\left(\mathrm{PPh}_{3}\right)_{2} \mathrm{Ru}(\mathrm{H})\left(\mathrm{L}^{8}\right)(\mathrm{CO})(\mathbf{2 9}),(\mathbf{f})\left(\mathrm{PPh}_{3}\right)_{2} \mathrm{Ru}(\mathrm{C}(\mathrm{H})=\mathrm{C}(\mathrm{H}) \mathrm{BMIDA})\left(\mathrm{L}^{24}\right)(\mathrm{CO})$ in 33, (g) $\left(\mathrm{PPh}_{3}\right)_{2} \mathrm{Ru}\left(\mathrm{C}(\mathrm{C} \equiv \mathrm{C}(\mathrm{Ph})=\mathrm{C}(\mathrm{H}) \mathrm{Ph})\left(\mathrm{L}^{84}\right)\right.$ (CO) (38), (h) $\left(\mathrm{PPh}_{3}\right)_{2} \mathrm{Ru}(\mathrm{X})\left(\mathrm{L}^{24}\right)(\mathrm{CO})(43)$, (i) $\left(\mathrm{PPh}_{3}\right)_{2} \mathrm{Ru}\left(\mathrm{L}^{24}\right)(\mathrm{CO}) \mathrm{Cl}$ in 44 , (j) $\left(\mathrm{PPh}_{3}\right) \mathrm{Ru}\left(\mathrm{Ge}(\mathrm{p}-\mathrm{tolyl})_{3}\right)\left(\mathrm{L}^{24}\right)(\mathrm{CO})_{2}(48)$, and (k) $\mathrm{Ru}\left(\mathrm{L}^{22}\right)(\mathrm{CO})_{3} \mathrm{Cl}(49)$. In $(\mathbf{b}, \mathbf{c}, \mathbf{g}, \mathbf{h})$, only the ispo-C atoms of the phosphane bound phenyl rings are shown for clarity. Additional color code: silicon, dark green; boron, teal.

Among the $\mathrm{Cp}^{*}$ structures, a new motif is noted for 56 [63] and 57 [64], with that of cationic 57, with a dissymmetric dithiocarbamate ligand, illustrated in Figure 5d. Here, the ruthenium is chelated by two dithiocarbamates and is capped by a Cp* resulting in, formally, a seven coordinate geometry based on a piano stool. A similar geometry is formed in neutral 58 [65], Figure 5e, and 59 [64] where one dithiocarbamate ligand has been replaced by two chlorides. Similar neutral species are seen in 60 [64] and 61 [64], Figure $5 f$, where the one dithiocarbamate ligand of 56 has been substituted by dinegative dithiocarbonate. The geometries of the cations in 62 [64] and 63 [64], resembling that seen in Figure $5 c$ for 55 whereby the carbonyl has been substituted for a chloride. A variation is observed in 64 [65] as dimerization has occurred leading to the dicationic species shown in Figure $5 \mathrm{~g}$ and a coordination geometry based on a piano stool. A piano stool geometry is also noted for dicationic 65 [65]. The molecule in 66 [64] is the $C \mathrm{p}^{*}$ analogue of 55 shown in Figure 5c. 


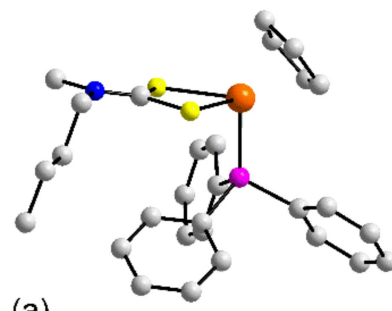

(a)

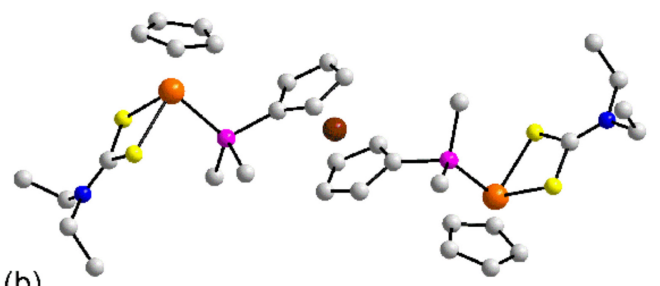

(b)

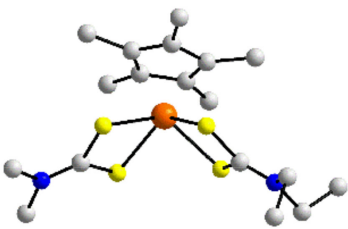

(d)

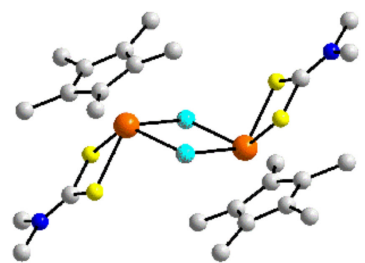

(g)

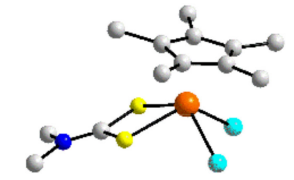

(e)

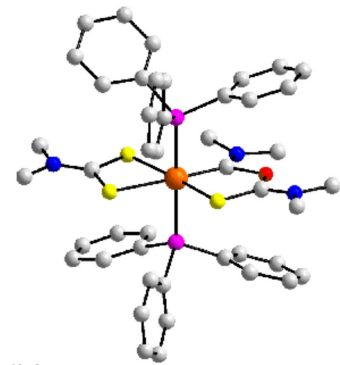

(h)

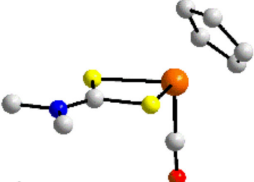

(c)

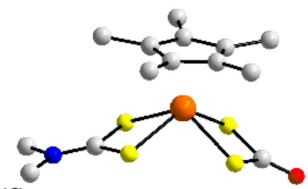

(f)

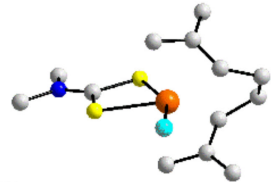

(i)

Figure 5. Molecular structures of ruthenium complexes: (a) $\mathrm{CpRu}\left(\mathrm{PPh}_{3}\right)\left(\mathbf{L}^{11}\right)(\mathbf{5 0})$, (b) $\left\{\left[\mathrm{CpRu}\left(\mathrm{L}^{\mathbf{2 4}}\right)\right]_{2}(\mathrm{dppf})\right\}$ in the crystal of 54, (c) $\mathrm{CpRu}\left(\mathbf{L}^{8}\right)(\mathrm{CO})(55)$, (d) $\left[\mathrm{Cp} * \mathrm{Ru}\left(\mathbf{L}^{8}\right)\left(\mathbf{L}^{24}\right)\right]^{+}$in 57, (e) $\mathrm{Cp}^{*} \mathrm{Ru}\left(\mathrm{L}^{8}\right) \mathrm{Cl}_{2}$ (58), (f) $\mathrm{Cp} \mathrm{p}^{*} \mathrm{Ru}\left(\mathrm{S}_{2} \mathrm{C}=\mathrm{O}\right)\left(\mathrm{L}^{8}\right)(60)$, (g) $\left[\mathrm{Cp} * \mathrm{Ru}\left(\mathrm{L}^{8}\right) \mathrm{Cl}\right]_{2}{ }^{2+}$ in 64 , (h) $\left[\left(\mathrm{PPh}_{3}\right)_{2} \mathrm{Ru}\left(\mathrm{C}\left(\mathrm{NMe}_{2}\right) \mathrm{OC}\left(\mathrm{NMe}_{2}\right)=\mathrm{S}\right)\left(\mathrm{L}^{8}\right)\right]^{+}$in 67 , and $(\mathbf{i}) \mathrm{Ru}\left(\mathrm{X}^{1}\right)\left(\mathrm{L}^{8}\right) \mathrm{Cl}(69)$. In (b), only the ispo-C atoms of the phosphane bound phenyl rings are shown for clarity.

The remaining structures to be described may be considered miscellaneous organometallic species as they contain neither carbonyl nor cyclopentadienyl type ligands. For the cations in 67 [43], Figure 5h, and 68 [43], the ruthenium is chelated by a dithiocarbamate as well as $C, N$-chelated by the $C\left(\mathrm{NMe}_{2}\right) X C\left(\mathrm{NMe}_{2}\right)=S, X=\mathrm{O}(67), S(68)$, ligand with the octahedral geometry completed by two trans oriented phosphane ligands. Cationic structure 68 was derived from 18 whereby 18 was treated with one molar equivalent of $\left[\mathrm{M}(\mathrm{MeCN})_{4}\right]\left[\mathrm{ClO}_{4}\right](\mathrm{M}=\mathrm{Cu}, \mathrm{Ag})$ which resulted in C-S bond cleavage of $\mathbf{L}^{8}$ and reassembly with another $\mathbf{L}^{8}$ anion to generate the $C, S$ chelating ligand. Prolonged standing resulted in hydrolysis and the ligand shown in Figure $5 \mathrm{~h}$. The common feature of 69 [66], Figure 5i, 70 [66], and 71 [66] is the presence of the di-allyl type ligand, $\left(\eta^{3}\right.$, $\eta^{3-}$ 2,7-dimethylocta-1,6-dien-3,8-diyl), which is assumed. Finally, the molecule in 72 [67], with a p-cymene ligand, assumes a coordination geometry resembling that of in 55 in Figure 5c.

As mentioned in the Introduction, crystals of metal dithiocarbamates are prone to display assemblies featuring $\mathrm{C}-\mathrm{H} \cdots \pi\left(\right.$ metal- $\left._{2} \mathrm{C}\right)$ interactions [15-17]. While the focus of the present overview is upon molecular geometries, it is worth highlighting some examples drawn from the ruthenium dithiocarbamates 1-72 where $\mathrm{C}-\mathrm{H} \cdots \pi\left(\mathrm{RuS}_{2} \mathrm{C}\right)$ interactions are evident in their crystals. In Figure $6 \mathrm{a}$, a supramolecular dimer is formed in the crystal of 3 through intermolecular $\mathrm{C}-\mathrm{H} \cdots \pi\left(\mathrm{RuS}_{2} \mathrm{C}\right)$ interactions occurring between centrosymmetrically related molecules. A similar aggregate is formed in the crystal of $\mathbf{8}$ despite the presence of bulky phosphane ligands. Supramolecular chains are also evident, for example in the crystal of $\mathbf{1}$ where a zigzag chain is evident with each independent molecule forming 
on average two $\mathrm{C}-\mathrm{H} \cdots \pi\left(\mathrm{RuS}_{2} \mathrm{C}\right)$ interactions. In the crystal of 58 , a helical chain features $\mathrm{C}-\mathrm{H} \cdots \pi\left(\mathrm{RuS}_{2} \mathrm{C}\right)$ interactions.

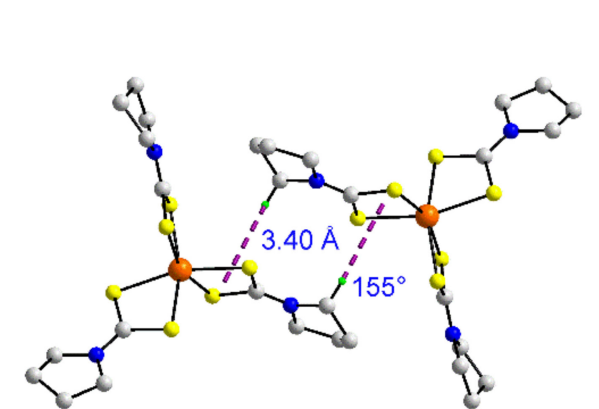

(a)

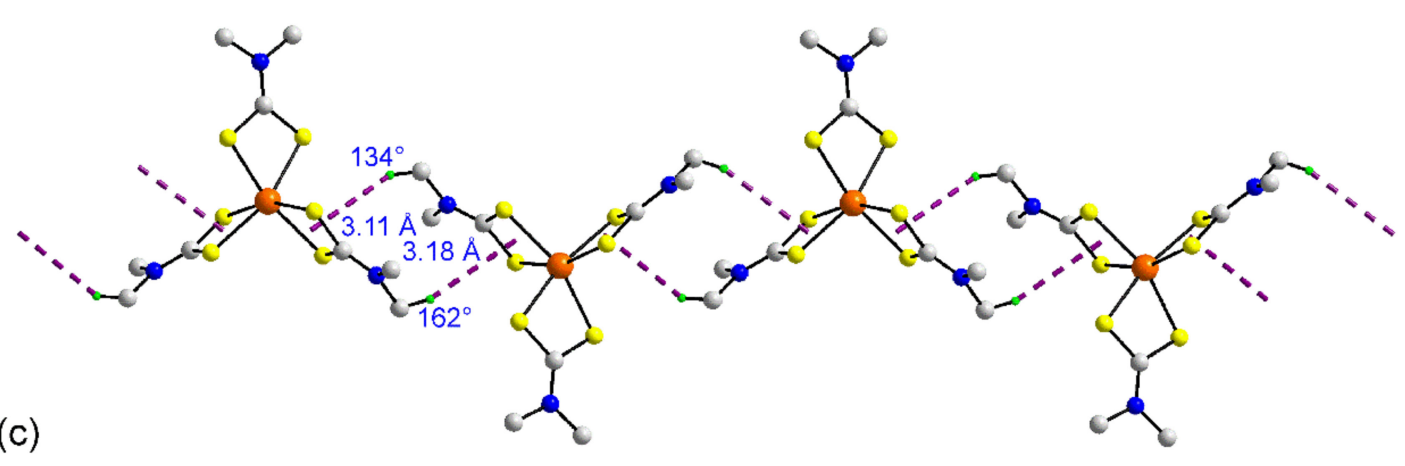

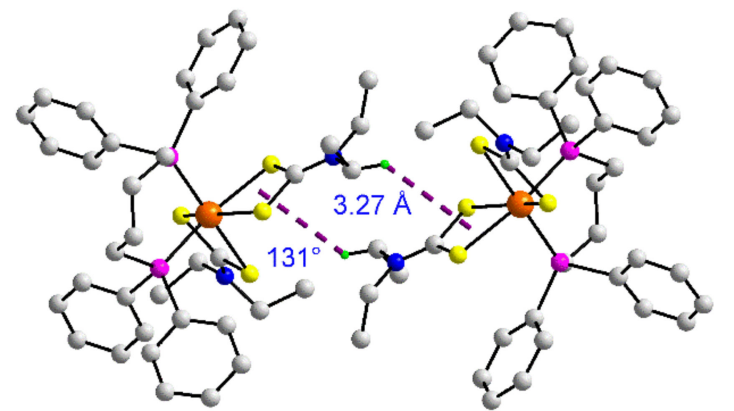

(b)

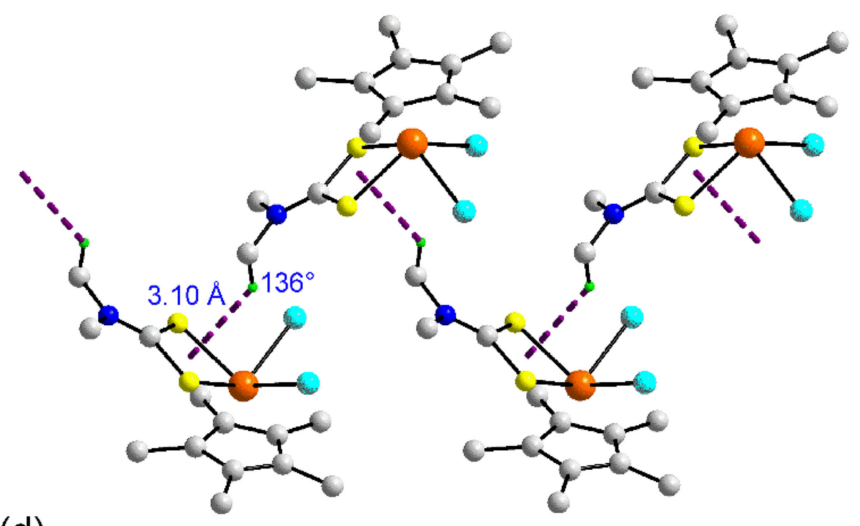

(d)

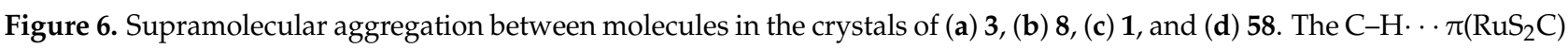
interactions are represented by dashed purple lines, and the distances and angles associated with the $\mathrm{C}-\mathrm{H} \cdots \pi\left(\mathrm{RuS}{ }_{2} \mathrm{C}\right)$ interactions are given on each image.

\subsubsection{Rhodium}

As summarized in Table 3, there are five known rhodium dithiocarbamate structures reported in the timeframe of this review.

Table 3. Summary of rhodium dithiocarbamate structures established by X-ray crystallography. See Table 1, Figure 2, and the Abbreviations section.

\begin{tabular}{clccc}
\hline Crystal & \multicolumn{1}{c}{ Formulation } & REFCODE & Donor Set & Ref. \\
\hline 73 & $\mathrm{Tp} * \mathrm{Rh}\left(\eta^{2}-\mathrm{L}^{\mathbf{2 4}}\right)\left(\eta^{1}-\mathrm{L}^{\mathbf{2 4}}\right)$ & BILHIO & $\mathrm{N}_{3} \mathrm{~S}_{3}$ & {$[68]$} \\
74 & {$\left[\mathrm{Rh}(\mathrm{H})\left\{\mathrm{k}^{3}-\mathrm{P}, \mathrm{Si}_{1} \mathrm{P}^{\prime}-\mathrm{SiPh}\left(\mathrm{NCH}_{2} \mathrm{PPh}_{2}\right)_{2} \mathrm{C}_{6} \mathrm{H}_{4}\right\}\left(\mathbf{L}^{24}\right)\right], 0.5\left(\mathrm{C}_{6} \mathrm{H}_{6}\right)$} & WODHOO & $\mathrm{HP}_{2} \mathrm{~S}_{2} \mathrm{Si}$ & {$[69]$} \\
75 & $\mathrm{Rh}(\mathrm{phpy})_{2}\left(\mathrm{~L}^{24}\right)$ & EMOKAU & $\mathrm{C}_{2} \mathrm{~N}_{2} \mathrm{~S}_{2}$ & {$[70]$} \\
76 & $\mathrm{Rh}(\mathrm{phpy})_{2}\left(\mathbf{L}^{66}\right)$ & XOCFUS & $\mathrm{C}_{2} \mathrm{~N}_{2} \mathrm{~S}_{2}$ & {$[71]$} \\
77 & $(\mathrm{PMe})_{2} \mathrm{Rh}\left(\mathrm{X}^{2}\right)\left(\mathrm{L}^{24}\right)$ & KUPVOH & $\mathrm{C}_{2} \mathrm{P}_{2} \mathrm{~S}_{2}$ & {$[72]$} \\
\hline
\end{tabular}


In keeping with the predominance of octahedral coordination geometries about the rhodium dithiocarbamate structures in 73-77, in 73 [68], one of the dithiocarbamate ligands adopts a relatively rare monodentate mode of coordination while the other retains its usual chelating mode. The three remaining positions in the octahedral geometry are occupied by the nitrogen atoms of the tripodal $\mathrm{Tp} \mathrm{p}^{*}$ ligand, Figure $7 \mathrm{a}$. Figure $7 \mathrm{~b}$ gives a representation of the hydride in 74 [69]. Stabilization to the molecule is provided by the tripodal ligand which coordinates by the central silicon atom and the two phosphorus atoms leading to a $\mathrm{HP}_{2} \mathrm{~S}_{2} \mathrm{Si}$ donor set as the dithiocarbamate ligand is coordinating in the anticipated chelating mode. The molecules in 75 [70], Figure 7c, and 76 [71], are closely related with the rhodium atom being coordinated by a chelating dithiocarbamate ligand and two C,Nchelating 2-(pyridin-2-yl)phenyl ligands with the nitrogen atoms being mutually trans. Finally, in 77 [72], Figure $7 d, a C_{2} P_{2} S_{2}$ donor set is apparent owing to the presence of a chelating 1,2-bis(2-phenylethynylmethylene)cyclohexane ligand, the phosphorus atoms are mutually trans.

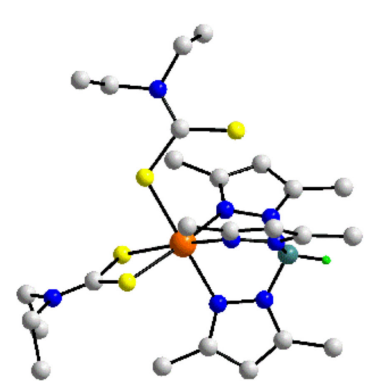

(a)

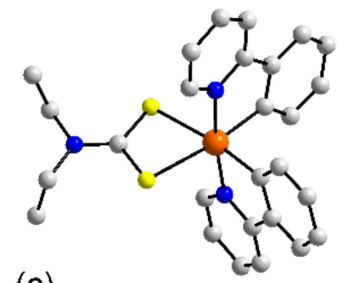

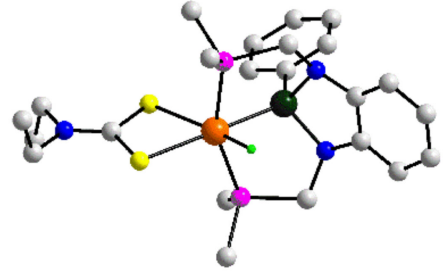

(b)

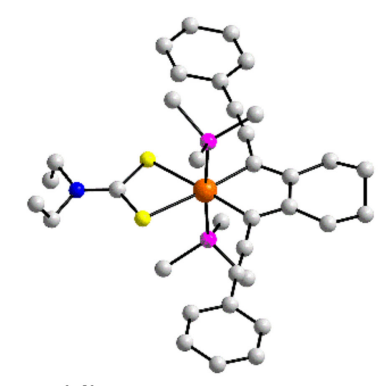

(d)

Figure 7. Molecular structures of rhodium complexes: (a) $\mathrm{Tp} \mathrm{p}^{*} \mathrm{Rh}\left(\eta^{2}-\mathrm{L}^{24}\right)\left(\eta^{1}-\mathrm{L}^{24}\right)(\mathbf{7 3}),(\mathbf{b})\left[\mathrm{Rh}(\mathrm{H})\left\{\mathrm{k}^{3}-\right.\right.$ $\left.\left.\mathrm{P}, \mathrm{Si}, \mathrm{P}^{\prime}-\mathrm{SiPh}\left(\mathrm{NCH}_{2} \mathrm{PPh}_{2}\right)_{2} \mathrm{C}_{6} \mathrm{H}_{4}\right\}\left(\mathrm{L}^{24}\right)\right]$ in the crystal of 74, (c) $\mathrm{Rh}(\mathrm{phpy})_{2}\left(\mathrm{~L}^{24}\right)(\mathbf{7 5})$, and (d) $\left(\mathrm{PMe}_{3}\right)_{2} \mathrm{Rh}\left(\mathrm{X}^{2}\right)$ $\left(\mathrm{L}^{24}\right)(77)$. In (b), only the ispo-C atoms of the phosphane bound phenyl rings are shown for clarity.

\subsubsection{Palladium}

A total of 79 distinct structures have been identified for palladium dithiocarbamates, Table 4. Despite the rather large number of structures, there is limited structural diversity among these so there are less than 10 distinct structural motifs in this category. The molecules contain palladium(II) centers and are generally considered four coordinate complexes within a square planar coordination geometry; in all cases the dithiocarbamate ligand functions as a $\mathrm{k}^{2}$ ligand. This is exemplified for the binary complexes of the general formula $\operatorname{Pd}\left(\mathrm{L}^{\mathbf{n}}\right)_{2}: \mathbf{7 8}$ [73], 79 [74-76], 80 [77], 81 [78], 82 [79], 83 [80], 84 [81], 85 [82], 86 [83], 87 [84], 88 [79], 89 [78], 90 [79], 91 [85], 92 [86], 93 [87], 94 [79], 95 [88], 96 [89], 97 [90], and 98 [91]. A representative molecule for 78-98 is that of $\operatorname{Pd}\left(\mathbf{L}^{13}\right)_{2}(\mathbf{8 1})$ shown in Figure 8a. Here, the palladium atom is bis-chelated by two dithiocarbamate ligands resulting in a square planar geometry defined by a $S_{4}$ donor set. The next three binary palladium dithiocarbamates are constructed with multifunctional dithiocarbamate ligands, that is, bearing two $\mathrm{CS}_{2}{ }^{-}$functionalities. Thus, in 99 [92], 100 [92], Figure 8b, and 101 [93] two palladium atoms are bridged by two such ligands giving rise to the anticipated $\mathrm{S}_{4}$ donor sets, the aggregates in $\mathbf{9 9}$ and $\mathbf{1 0 1}$ are disposed about a center of inversion. The final 
binary complex is exceptional in that the polyfunctional dithiocarbamate ligand bears two phosphane donors. As shown in Figure 8c for the dication in the crystal of 102 [94], each dithiocarbamate ligand chelates one palladium atom via the sulfur atoms and the second palladium atoms via the phosphane atoms giving rise to a centrosymmetric aggregate and $\mathrm{P}_{2} \mathrm{~S}_{2}$ donor sets.

Table 4. Summary of palladium dithiocarbamate structures established by X-ray crystallography. See Table 1, Figure 2, and the Abbreviations section.

\begin{tabular}{|c|c|c|c|c|}
\hline Crystal & Formulation & REFCODE & Donor Set & Ref. \\
\hline 78 & $\operatorname{Pd}\left(\mathbf{L}^{4}\right)_{2}$ & YIRSIE & $\mathrm{S}_{4}$ & [73] \\
\hline 79 & $\operatorname{Pd}\left(\mathbf{L}^{8}\right)_{2}$ & OMUJEN01 * & $\mathrm{S}_{4}$ & [74-76] \\
\hline 80 & $\operatorname{Pd}\left(\mathbf{L}^{11}\right)_{2}$ & ODOYAI & $\mathrm{S}_{4}$ & [77] \\
\hline 81 & $\operatorname{Pd}\left(\mathbf{L}^{\mathbf{1 3}}\right)_{2}$ & LIGSAW & $\mathrm{S}_{4}$ & [78] \\
\hline 82 & $\operatorname{Pd}\left(\mathbf{L}^{15}\right)_{2}$ & ZOJVAX & $\mathrm{S}_{4}$ & [79] \\
\hline 83 & $\operatorname{Pd}\left(\mathbf{L}^{16}\right)_{2}$ & FUHXEN & $\mathrm{S}_{4}$ & [80] \\
\hline 84 & $\operatorname{Pd}\left(\mathbf{L}^{24}\right)_{2}$ & DETCPD02 & $\mathrm{S}_{4}$ & [81] \\
\hline 85 & $\operatorname{Pd}\left(\mathbf{L}^{25}\right)_{2}$ & FEVCER & $\mathrm{S}_{4}$ & [82] \\
\hline 86 & $\operatorname{Pd}\left(\mathbf{L}^{30}\right)_{2}$ & QOWXUW & $\mathrm{S}_{4}$ & [83] \\
\hline 87 & $\operatorname{Pd}\left(\mathbf{L}^{33}\right)_{2}$ & EMOKOI & $\mathrm{S}_{4}$ & [84] \\
\hline 88 & $\operatorname{Pd}\left(\mathbf{L}^{35}\right)_{2}$ & ZOJTUP & $\mathrm{S}_{4}$ & [79] \\
\hline 89 & $\operatorname{Pd}\left(\mathbf{L}^{39}\right)_{2}$ & LIGSEA & $\mathrm{S}_{4}$ & [78] \\
\hline 90 & $\operatorname{Pd}\left(\mathrm{L}^{39}\right)_{2}, \mathrm{C}_{5} \mathrm{H}_{5} \mathrm{~N}$ & ZOJTID & $\mathrm{S}_{4}$ & [79] \\
\hline 91 & $\operatorname{Pd}\left(\mathrm{L}^{39}\right)_{2}, 2 \mathrm{Cr}\left(\mathrm{C}_{6} \mathrm{H}_{6}\right)_{2}, 2\left(\mathrm{C}_{60}\right)$ & CEBCES & $\mathrm{S}_{4}$ & [85] \\
\hline 92 & $\operatorname{Pd}\left(\mathbf{L}^{41}\right)_{2}$ & HOMXEO & $\mathrm{S}_{4}$ & [86] \\
\hline 93 & $\operatorname{Pd}\left(\mathrm{L}^{42}\right)_{2}$ & LUKTES & $\mathrm{S}_{4}$ & [87] \\
\hline 94 & $\mathrm{Pd}\left(\mathrm{L}^{47}\right)_{2}, \mathrm{C}_{5} \mathrm{H}_{5} \mathrm{~N}$ & ZOJTOJ & $\mathrm{S}_{4}$ & [79] \\
\hline 95 & $\operatorname{Pd}\left(\mathbf{L}^{57}\right)_{2}$ & UBIFUI & $\mathrm{S}_{4}$ & [88] \\
\hline 96 & $\operatorname{Pd}\left(\mathbf{L}^{63}\right)_{2}$ & XAZZOO & $\mathrm{S}_{4}$ & [89] \\
\hline 97 & $\operatorname{Pd}\left(\mathbf{L}^{64}\right)_{2}$ & JESJUN & $\mathrm{S}_{4}$ & {$[90]$} \\
\hline 98 & {$\left[\mathrm{Pd}\left(\mathrm{L}^{66}\right)_{2}\right], 3 \mathrm{H}_{2} \mathrm{O}$} & BEZKAU & $\mathrm{S}_{4}$ & [91] \\
\hline 99 & $\mathrm{Pd}_{2}\left(\mathrm{~L}^{86}\right)_{2}$ & KENYIN & $\mathrm{S}_{4}$ & [92] \\
\hline 100 & $\mathrm{Pd}_{2}\left(\mathbf{L}^{86}\right)_{2}, \mathrm{CHCl}_{3}$ & KENYOT & $\mathrm{S}_{4}$ & [92] \\
\hline 101 & $\mathrm{Pd}_{2}\left(\mathrm{~L}^{87}\right)_{2}, 2\left(\mathrm{CH}_{3} \mathrm{OH}\right)$ & IXIWAN & $\mathrm{S}_{4}$ & [93] \\
\hline 102 & {$\left[\mathrm{Pd}_{2}\left(\mathrm{~L}^{44}\right)_{2}\right] \mathrm{Cl}_{2}, 1.5\left(\mathrm{CH}_{3} \mathrm{OH}\right), 2\left(\mathrm{H}_{2} \mathrm{O}\right)$} & IGUBAP & $\mathrm{P}_{2} \mathrm{~S}_{2}$ & [94] \\
\hline 103 & $($ fcdpm $) \operatorname{Pd}\left(\mathbf{L}^{24}\right)$ & YEFVOW & $\mathrm{N}_{2} \mathrm{~S}_{2}$ & [95] \\
\hline 104 & $($ fcdpm $) \operatorname{Pd}\left(\mathbf{L}^{29}\right)$ & YEFVUC & $\mathrm{N}_{2} \mathrm{~S}_{2}$ & [95] \\
\hline 105 & $($ fcdpm $) \operatorname{Pd}\left(\mathbf{L}^{43}\right)$ & VIZJOF & $\mathrm{N}_{2} \mathrm{~S}_{2}$ & [96] \\
\hline 106 & {$\left[\mathrm{Pd}(\mathrm{dmbp})\left(\mathrm{L}^{75}\right)\right]\left[\mathrm{PF}_{6}\right]_{2}$} & XEDWOV & $\mathrm{N}_{2} \mathrm{~S}_{2}$ & [97] \\
\hline 107 & {$\left[\mathrm{Pd}(\mathrm{bpy})\left(\mathrm{L}^{75}\right)\right]\left[\mathrm{PF}_{6}\right]_{2}, \mathrm{CH}_{3} \mathrm{CN}$} & XEDWUB & $\mathrm{N}_{2} \mathrm{~S}_{2}$ & [97] \\
\hline 108 & {$\left[(\right.$ dppe $\left.) \mathrm{Pd}\left(\mathrm{L}^{57}\right)\right] \mathrm{Cl}, 1.33\left(\mathrm{H}_{2} \mathrm{O}\right), \mathrm{CHCl}_{3}$} & DIFQIU & $\mathrm{P}_{2} \mathrm{~S}_{2}$ & [26] \\
\hline 109 & {$\left[(\mathrm{dppp}) \mathrm{Pd}\left(\mathrm{L}^{24}\right)\right] \mathrm{BF}_{4}, 2\left(\mathrm{CHCl}_{3}\right)$} & WUMQEB & $\mathrm{P}_{2} \mathrm{~S}_{2}$ & [98] \\
\hline 110 & {$\left[(\mathrm{dppf}) \mathrm{Pd}\left(\mathbf{L}^{57}\right)\right] \mathrm{PF}_{6}, \mathrm{CH}_{2} \mathrm{Cl}_{2}$} & DOZKOV & $\mathrm{P}_{2} \mathrm{~S}_{2}$ & [99] \\
\hline 111 & $\left\{\left[\left(\mathrm{PPh}_{3}\right)_{2} \mathrm{Pd}\right]_{2}\left(\mathbf{L}^{74}\right)\right\}\left[\mathrm{PF}_{6}\right]_{2},\left(\mathrm{CH}_{3} \mathrm{CH}_{2}\right)_{2} \mathrm{O}$ & KEKKAP & $\mathrm{P}_{2} \mathrm{~S}_{2}$ & [100] \\
\hline 112 & $\left\{\left[\left(\mathrm{PPh}_{3}\right)_{2} \mathrm{Pd}\right]_{2}\left(\mathrm{~L}^{74}\right)\right\}\left[\mathrm{PF}_{6}\right]_{2},\left(\mathrm{CH}_{3} \mathrm{CH}_{2}\right)_{2} \mathrm{O}$ & KEKKET & $\mathrm{P}_{2} \mathrm{~S}_{2}$ & [100] \\
\hline 113 & $\left\{\left[\left(\mathrm{PPh}_{3}\right)_{2} \mathrm{Pd}\right]_{2}\left(\mathrm{~L}^{85}\right)\right\}\left[\mathrm{PF}_{6}\right]_{2},\left(\mathrm{CH}_{3} \mathrm{CH}_{2}\right)_{2} \mathrm{O}$ & KEKKIX & $\mathrm{P}_{2} \mathrm{~S}_{2}$ & [100] \\
\hline 114 & $\left\{[(\mathrm{dppf}) \mathrm{Pd}]_{2}\left(\mathrm{~L}^{74}\right)\right\}\left[\mathrm{BF}_{4}\right]_{2}, 2.6\left(\mathrm{CH}_{2} \mathrm{Cl}_{2}\right)$ & RUDLEI & $\mathrm{P}_{2} \mathrm{~S}_{2}$ & [101] \\
\hline 115 & $\left\{[(\mathrm{dppf}) \mathrm{Pd}]_{2}\left(\mathrm{~L}^{85}\right)\right\}\left[\mathrm{PF}_{6}\right]_{2}, \mathrm{CH}_{2} \mathrm{Cl}_{2}, \mathrm{CH}_{3} \mathrm{CH}_{2} \mathrm{OH}$ & ISEWIN & $\mathrm{P}_{2} \mathrm{~S}_{2}$ & [102] \\
\hline 116 & $\left(\mathrm{PPh}_{3}\right) \mathrm{Pd}\left(\mathrm{L}^{31}\right) \mathrm{Cl}$ & RASYAN & $\mathrm{ClPS}_{2}$ & [103] \\
\hline 117 & $\left(\mathrm{PPh}_{3}\right) \mathrm{Pd}\left(\mathrm{L}^{32}\right) \mathrm{Cl}$ & NAJVEC & $\mathrm{ClPS}_{2}$ & {$[104]$} \\
\hline 118 & $\left(\mathrm{PPh}_{3}\right) \mathrm{Pd}\left(\mathrm{L}^{47}\right) \mathrm{Cl}$ & KIJROL & $\mathrm{ClPS}_{2}$ & [105] \\
\hline 119 & $\left(\mathrm{PPh}_{3}\right) \mathrm{Pd}\left(\mathbf{L}^{57}\right) \mathrm{Cl}, 0.5\left(\mathrm{CHCl}_{3}\right)$ & DICXOE & $\mathrm{ClPS}_{2}$ & [26] \\
\hline 120 & $\left(\mathrm{PPh}_{3}\right) \mathrm{Pd}\left(\mathrm{L}^{64}\right) \mathrm{Cl}$ & SAYZOI & $\mathrm{ClPS}_{2}$ & [106] \\
\hline 121 & $\left(\mathrm{PPh}_{3}\right) \mathrm{Pd}\left(\mathrm{L}^{68}\right) \mathrm{Cl}, 0.5\left(\mathrm{CHCl}_{3}\right)$ & NAJNOE & $\mathrm{ClPS}_{2}$ & [104] \\
\hline
\end{tabular}


Table 4. Cont.

\begin{tabular}{|c|c|c|c|c|}
\hline Crystal & Formulation & REFCODE & Donor Set & Ref. \\
\hline 122 & $\left(\mathrm{PPh}_{3}\right) \mathrm{Pd}\left(\mathbf{L}^{81}\right) \mathrm{Cl}$ & BULBAM & $\mathrm{ClPS}_{2}$ & [107] \\
\hline 123 & {$\left[\mathrm{P}(\text { o-tolyl })_{3}\right] \mathrm{Pd}\left(\mathbf{L}^{\mathbf{8 1}}\right) \mathrm{Cl}$} & BUKZUD * & $\mathrm{ClPS}_{2}$ & {$[107,108]$} \\
\hline 124 & {$\left[\mathrm{P}(\text { p-tolyl })_{3}\right] \mathrm{Pd}\left(\mathbf{L}^{39}\right) \mathrm{Cl}$} & VANWAL & $\mathrm{ClPS}_{2}$ & [109] \\
\hline 125 & {$\left[\mathrm{P}(\mathrm{p} \text {-tolyl })_{3}\right] \mathrm{Pd}\left(\mathrm{L}^{69}\right) \mathrm{Cl}$} & ROHJUW & $\mathrm{ClPS}_{2}$ & [110] \\
\hline 126 & {$\left[\mathrm{P}(\mathrm{p} \text {-tolyl })_{3}\right] \mathrm{Pd}\left(\mathrm{L}^{71}\right) \mathrm{Cl}, \mathrm{CHCl}_{3}$} & UNOYAZ & $\mathrm{ClPS}_{2}$ & [111] \\
\hline 127 & {$\left[\mathrm{P}(\mathrm{p} \text {-tolyl })_{3}\right] \mathrm{Pd}\left(\mathrm{L}^{76}\right) \mathrm{Cl}$} & ROHKAD & $\mathrm{ClPS}_{2}$ & [110] \\
\hline 128 & {$\left[\mathrm{P}\left(\mathrm{C}_{6} \mathrm{H}_{4} \mathrm{Cl}-\mathrm{p}\right)_{3}\right] \mathrm{Pd}\left(\mathrm{L}^{69}\right) \mathrm{Cl}$} & ADUHAL & $\mathrm{ClPS}_{2}$ & [112] \\
\hline 129 & {$\left[\mathrm{P}\left(\mathrm{C}_{6} \mathrm{H}_{4} \mathrm{Cl}-\mathrm{p}\right)_{3}\right] \mathrm{Pd}\left(\mathbf{L}^{72}\right) \mathrm{Cl}$} & MEHHIT & $\mathrm{ClPS}_{2}$ & [113] \\
\hline 130 & {$\left[\mathrm{P}\left(\mathrm{C}_{6} \mathrm{H}_{4} \mathrm{Cl}-\mathrm{p}\right)_{3}\right] \mathrm{Pd}\left(\mathbf{L}^{77}\right) \mathrm{Cl}$} & QEKTAE & $\mathrm{ClPS}_{2}$ & [114] \\
\hline 131 & {$\left[\mathrm{P}\left(\mathrm{C}_{6} \mathrm{H}_{4} \mathrm{~F}-\mathrm{p}\right)\right] \mathrm{Pd}\left(\mathrm{L}^{69}\right) \mathrm{Cl}$} & ADUGOY & $\mathrm{ClPS}_{2}$ & [112] \\
\hline 132 & {$\left[\mathrm{P}\left(\mathrm{C}_{6} \mathrm{H}_{4} \mathrm{~F}-\mathrm{p}\right)\right] \mathrm{Pd}\left(\mathrm{L}^{71}\right) \mathrm{Cl}$} & ADUGUE & $\mathrm{ClPS}_{2}$ & [112] \\
\hline 133 & {$\left[\mathrm{P}\left(\mathrm{C}_{6} \mathrm{H}_{4} \mathrm{~F}-\mathrm{p}\right)\right] \mathrm{Pd}\left(\mathbf{L}^{72}\right) \mathrm{Cl}$} & MEHHEP & $\mathrm{ClPS}_{2}$ & [113] \\
\hline 134 & $\left.\left[\mathrm{P}\left(\mathrm{C}_{6} \mathrm{H}_{4} \mathrm{~F}-\mathrm{p}\right)\right] \mathrm{Pd}\left(\mathrm{L}^{77}\right) \mathrm{Cl}\right\} 0.5\left(\mathrm{CH}_{3} \mathrm{COCH}_{3}\right)$ & QEKSUX & $\mathrm{ClPS}_{2}$ & [114] \\
\hline 135 & {$\left[\mathrm{PPh}_{2}(\right.$ o-tolyl $\left.)\right] \mathrm{Pd}\left(\mathbf{L}^{71}\right) \mathrm{Cl}$} & GIQGUK & $\mathrm{ClPS}_{2}$ & [115] \\
\hline 136 & {$\left[\mathrm{PPh}_{2}(\right.$ p-tolyl $\left.)\right] \mathrm{Pd}\left(\mathrm{L}^{31}\right) \mathrm{Cl}$} & RASYER & $\mathrm{ClPS}_{2}$ & [103] \\
\hline 137 & {$\left[\mathrm{PPh}_{2}(\mathrm{p}\right.$-tolyl $\left.)\right] \mathrm{Pd}\left(\mathrm{L}^{39}\right) \mathrm{Cl}$} & VANVUE & $\mathrm{ClPS}_{2}$ & [109] \\
\hline 138 & {$\left[\mathrm{PPh}_{2}(\mathrm{p}\right.$-tolyl $\left.)\right] \mathrm{Pd}\left(\mathrm{L}^{67}\right) \mathrm{Cl}$} & VANVOY & $\mathrm{ClPS}_{2}$ & [109] \\
\hline 139 & {$\left[\mathrm{PPh}_{2}(\mathrm{p}\right.$-tolyl $\left.)\right] \mathrm{Pd}\left(\mathrm{L}^{69}\right) \mathrm{Cl}$} & ROHJOQ & $\mathrm{ClPS}_{2}$ & [110] \\
\hline 140 & {$\left[\mathrm{PPh}_{2}(\mathrm{p}\right.$-tolyl $\left.)\right] \mathrm{Pd}\left(\mathbf{L}^{71}\right) \mathrm{Cl}$} & UNOYON & $\mathrm{ClPS}_{2}$ & [111] \\
\hline 141 & {$\left[\mathrm{PPh}_{2}\left(\mathrm{C}_{6} \mathrm{H}_{4} \mathrm{OMe}-\mathrm{O}\right)\right] \mathrm{Pd}\left(\mathbf{L}^{24}\right) \mathrm{Cl}, \mathrm{H}_{2} \mathrm{O}$} & RAFLER & $\mathrm{ClPS}_{2}$ & [116] \\
\hline 142 & {$\left[\mathrm{PPh}_{2}(\mathrm{nPr})\right] \mathrm{Pd}\left(\mathrm{L}^{26}\right) \mathrm{Cl}$} & EHOBOU & $\mathrm{ClPS}_{2}$ & [117] \\
\hline 143 & {$\left[\mathrm{PPh}_{2}(\mathrm{tBu})\right] \mathrm{Pd}\left(\mathrm{L}^{38}\right) \mathrm{Cl}$} & EHOBUA & $\mathrm{ClPS}_{2}$ & [117] \\
\hline 144 & {$\left[\mathrm{PPh}_{2}\left(\mathrm{CH}_{2} \mathrm{Ph}\right)\right] \mathrm{Pd}\left(\mathbf{L}^{8}\right) \mathrm{Cl}$} & RAFLAN & $\mathrm{ClPS}_{2}$ & [116] \\
\hline 145 & {$\left[\mathrm{PPh}_{2}\left(\mathrm{CH}_{2} \mathrm{Ph}\right)\right] \mathrm{Pd}\left(\mathrm{L}^{24}\right) \mathrm{Cl}$} & GIQHAR & $\mathrm{ClPS}_{2}$ & [115] \\
\hline 146 & {$\left[\left(\mathrm{PPh}_{3}\right) \mathrm{Pd}\left(\mathrm{L}^{68}\right)(\mathrm{SCN})\right], 0.5\left(\mathrm{CH}_{2} \mathrm{Cl}_{2}\right)$} & NAJNIY & $\mathrm{PS}_{3}$ & [104] \\
\hline 147 & {$[\mathrm{tBu}(\mathrm{Ph}) \mathrm{POH}][\mathrm{tBu}(\mathrm{Ph}) \mathrm{P}(=\mathrm{O})] \mathrm{Pd}\left(\mathrm{L}^{24}\right)$} & SAGBIM & $\mathrm{P}_{2} \mathrm{~S}_{2}$ & [118] \\
\hline 148 & $\mathrm{Cu}\left\{[\mathrm{tBu}(\mathrm{PhP})(\mathrm{O})] \mathrm{Pd}\left(\mathrm{L}^{24}\right)\right\}_{2}$ & SAFZUV & $\mathrm{P}_{2} \mathrm{~S}_{2}$ & [118] \\
\hline 149 & $\operatorname{Pd}(\mathrm{phpz})\left(\mathrm{L}^{24}\right)$ & UMEBEU & $\mathrm{CNS}_{2}$ & [119] \\
\hline 150 & $\operatorname{Pd}($ phpy $)\left(\mathbf{L}^{63}\right)$ & JUPNIT & $\mathrm{CNS}_{2}$ & [120] \\
\hline 151 & $\operatorname{Pd}\left(\mathrm{C}^{\wedge} \mathrm{N}\right)\left(\mathbf{L}^{24}\right)$ & OVEPOV & $\mathrm{CNS}_{2}$ & [121] \\
\hline 152 & $\operatorname{Pd}\left(X^{3}\right)\left(\mathbf{L}^{24}\right), \mathrm{CH}_{3}\left(\mathrm{CH}_{2}\right)_{4} \mathrm{CH}_{3}, 0.5\left(\mathrm{CH}_{2} \mathrm{Cl}_{2}\right)$ & FOHYEH & $\mathrm{CNS}_{2}$ & [122] \\
\hline 153 & $\mathrm{Pd}\left(\kappa^{2}-\mathrm{C}, \mathrm{N}-\mathrm{CH}_{2} \mathrm{SiPh}_{2}\left(\mathrm{CH}_{2} \mathrm{NC}_{5} \mathrm{H}_{10}\right)\right)\left(\mathrm{L}^{24}\right)$ & EZEXIQ & $\mathrm{CNS}_{2}$ & [123] \\
\hline 154 & $\left(\mathrm{PPh}_{3}\right) \operatorname{Pd}(\mathrm{pyr})\left(\mathrm{L}^{57}\right)$ & UQANEG & $\mathrm{CPS}_{2}$ & [124] \\
\hline 155 & $\mathrm{Pd}\left(\eta^{3}-\mathrm{C}_{4} \mathrm{H}_{7}\right)\left(\mathbf{L}^{8}\right)$ & ODOXOV & $\mathrm{C}_{2} \mathrm{~S}_{2}$ & [77] \\
\hline 156 & $\operatorname{Pd}\left(\eta^{3}-\mathrm{C}_{4} \mathrm{H}_{7}\right)\left(\mathrm{L}^{28}\right)$ & ODOYEM & $\mathrm{C}_{2} \mathrm{~S}_{2}$ & [77] \\
\hline
\end{tabular}

* CSD depositions are available for repeated measurements.

The ternary crystals 103 [95], 104 [95], 105 [96], 106 [97], and 107 [97], feature square planar $\mathrm{N}_{2} \mathrm{~S}_{2}$ donor sets defined by chelating dithiocarbamate and $\mathrm{N} \cap \mathrm{N}$ ligands. An example is illustrated for (fcdpm) $\mathrm{Pd}\left(\mathbf{L}^{43}\right)(\mathbf{1 0 5})$ in Figure $8 \mathrm{~d}$ which is notable for having both ligands bearing a ferrocenyl $(\mathrm{Fc})$ group. The next series of square planar complexes have $\mathrm{P}_{2} \mathrm{~S}_{2}$ donor sets with the phosphorus atoms derived from bidentate phosphane ligands in the mononuclear cations 108 [26], 109 [98], and 110 [99]. Bifunctional dithiocarbamates feature in complexes with monodentate phosphane ligands in dinuclear 111 [100], 112 [100], and 113 [100], and bidentate phosphanes in dinuclear 114 [101] and 115 [102]; a representative complex is shown for $\left\{\left[\left(\mathrm{PPh}_{3}\right)_{2} \mathrm{Pd}\right]_{2}\left(\mathbf{L}^{85}\right)\right\}^{2+}$ of $\mathbf{1 1 3}$ in Figure 8e. Quaternary molecules of the general formula $\left(\mathrm{PR}_{3}\right) \mathrm{Pd}\left(\mathrm{L}^{\mathbf{n}}\right) \mathrm{Cl}$ are found in 116 [103], 117 [104], 118 [105], 119 [26], 120 [106], 121 [104], 122 [107], 123 [107,108], 124 [109], 125 [110], 126 [111], 127 [110], $\mathbf{1 2 8}$ [112], 129 [113], 130 [114], 131 [112], 132 [112], 133 [113], 134 [114], 135 [115], 136 [103], 137 [109], 138 [109], 139 [110], 140 [111], 141 [116], 142 [117], 143 [117], 144 [116], and 145 [115]. The square planar coordination geometries have $\mathrm{ClPS}_{2}$ donor sets; a representative molecule is shown in Figure $8 \mathrm{f}$ for $\left(\mathrm{PPh}_{3}\right) \mathrm{Pd}\left(\mathbf{L}^{\mathbf{8 1}}\right) \mathrm{Cl}$ in $\mathbf{1 2 2}$. In $\mathbf{1 4 6}$ [104], the chloride has been replaced by a thiocyanate ligand leading to a $\mathrm{PS}_{3}$ donor set. The remaining structures in this category are deserving of special mention and/or are organometallic in nature. 
(a)

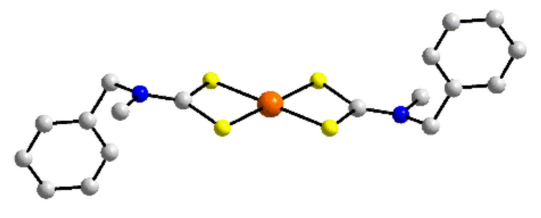

(b)
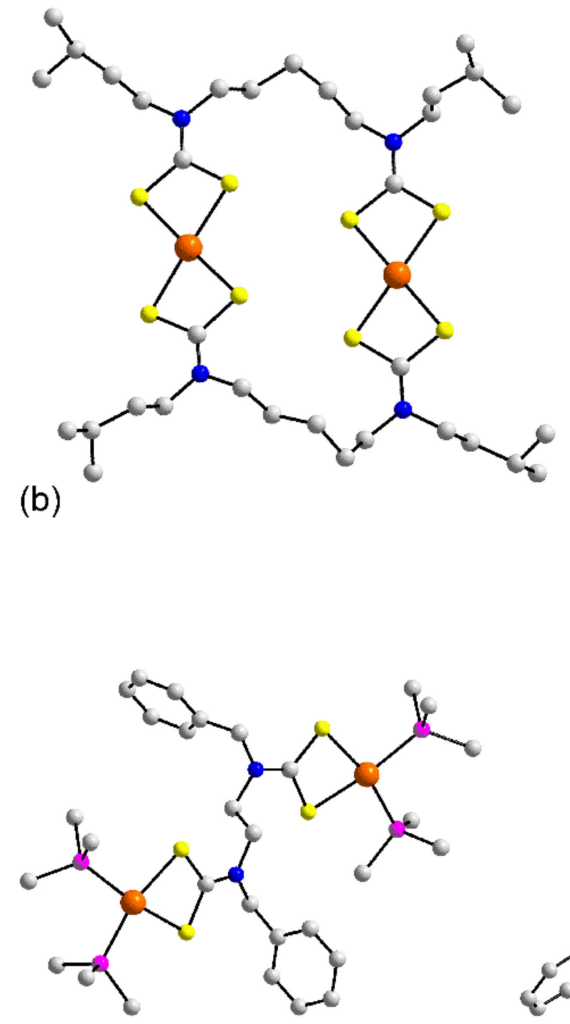

(e)

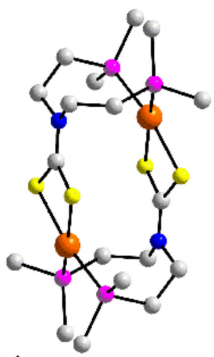

(c)

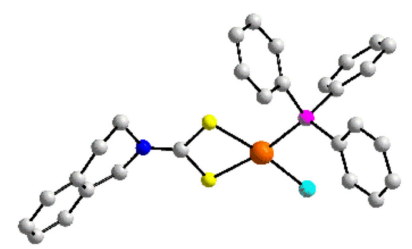

(f)

Figure 8. Molecular structures of palladium complexes: $(\mathbf{a}) \operatorname{Pd}\left(\mathbf{L}^{13}\right)_{2}(\mathbf{8 1}),(\mathbf{b}) \mathrm{Pd}_{2}\left(\mathbf{L}^{86}\right)_{2}$ in the crystal of $100,(\mathbf{c})\left[\mathrm{Pd}_{2}\left(\mathrm{~L}^{\mathbf{4 4}}\right)_{2}\right]^{2+}$ in 102, (d) (fcdpm) Pd $\left(\mathbf{L}^{43}\right)(\mathbf{1 0 5}),(\mathbf{e})\left\{\left[\left(\mathrm{PPh}_{3}\right)_{2} \mathrm{Pd}\right]_{2}\left(\mathrm{~L}^{85}\right)\right\}^{2+}$ in $\mathbf{1 1 3}$, and $(\mathbf{f})\left(\mathrm{PPh}_{3}\right) \mathrm{Pd}\left(\mathbf{L}^{81}\right) \mathrm{Cl}$ in 122 . In $(\mathrm{c}, \mathbf{e})$, only the ispo-C atoms of the phosphane bound phenyl rings are shown for clarity.

In the crystal of 147 [118], the neutral complex, Figure 9a, features a $\mathrm{P}_{2} \mathrm{~S}_{2}$ square planar geometry defined by, formally phosphane, $\mathrm{tBu}(\mathrm{Ph}) \mathrm{POH}$, and $\mathrm{P}$-bound phosphinoyl, $[\mathrm{tBu}(\mathrm{Ph}) \mathrm{PO}]^{-}$ligands as well as a chelating dithiocarbamate. However, in the crystal, the hydrogen atom is equally shared by the oxygen atoms, a feature of the molecule reflected in the equivalence of the Pd-P bond lengths. In $\mathbf{1 4 8}$ [118], Figure 9b, a similar coordination geometry is found with the palladium atom bound by two phosphinoyl-P atoms. A central copper(II) atom is also in a square planar geometry but defined by, formally, four oxido-O atoms. The complexes in 149 [119], 150 [120], 151 [121], 152 [122], and 153 [123] feature C,Nchelating ligands and $\mathrm{CNS}_{2}$ donor sets. An impressive example is that of 152, Figure 9c, being notable for the tethering, via cyclometallation, of the palladium dithiocarbamate complex to a nickel(II) porphyrin. Whereas $\mathbf{1 4 9 - 1 5 3}$ featured chelating C,N ligands, in the phosphane complex 154 [124], Figure $9 \mathrm{~d}$, the $\mathrm{CPS}_{2}$ donor set comprises monodentate phosphane and pyrimidin-5-yl ligands as well as the chelating dithiocarbamate ligand. The final two examples, that is 155 [77], Figure 9e, and 156 [77], formally feature $C_{2} S_{2}$ donor sets with the two coordination sites in addition to those provided by the dithiocarbamate ligand occupied by carbon atoms derived from the $\eta^{3-} 2$-methylallyl ligand. 


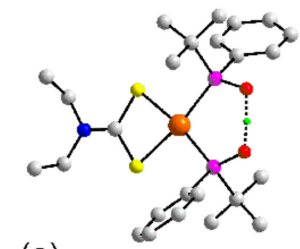

(a)

(b)

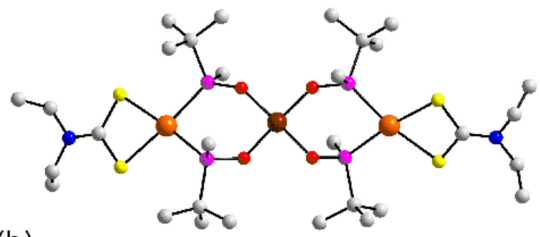

(b)

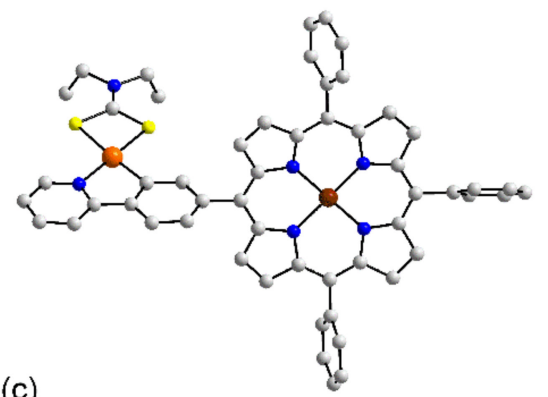

(c)

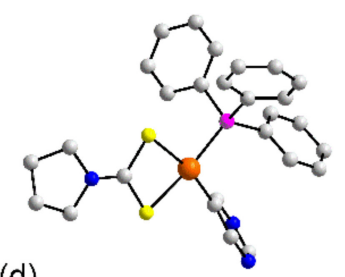

(d)

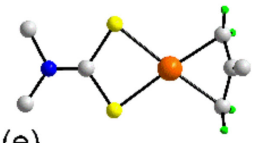

(e)

Figure 9. Molecular structures of palladium complexes: (a) $[\mathrm{tBu}(\mathrm{Ph}) \mathrm{POH}][\mathrm{tBu}(\mathrm{Ph}) \mathrm{P}(=\mathrm{O})] \mathrm{Pd}\left(\mathrm{L}^{24}\right)$ (147), (b) $\mathrm{Cu}\left\{[\mathrm{tBu}(\mathrm{PhP})(\mathrm{O})] \mathrm{Pd}\left(\mathrm{L}^{24}\right)\right\}_{2}(148),(\mathbf{c}) \mathrm{Pd}\left(\mathrm{X}^{3}\right)\left(\mathrm{L}^{24}\right)$ in 152, (d) $\left(\mathrm{PPh}_{3}\right) \mathrm{Pd}(\mathrm{pyr})\left(\mathrm{L}^{57}\right)(\mathbf{1 5 4})$, and (e) $\mathrm{Pd}\left(\eta^{3}-\mathrm{C}_{4} \mathrm{H}_{7}\right)\left(\mathbf{L}^{8}\right)$ (155). In (b), only the ispo-C atoms of the phosphane bound phenyl rings are shown for clarity.

\subsubsection{Osmium}

Nearly $80 \%$ of the structures identified for organometallic osmium(II) dithiocarbamates, crystals 157-176, are mainly contributed by Wright et al. over four years i.e., 2004 to 2007 with the primary motivation being interest in their reactivity [125-129]. The majority of the osmium centers have a coordination number six and exhibit octahedral geometry. Of note, all structures but $\mathbf{1 7 6}$ feature $\mathbf{L}^{\mathbf{8}}$; the exceptional structure, i.e., $\mathbf{1 7 6}$, has $\mathbf{L}^{\mathbf{2 4}}$. A list osmium dithiocarbamate structures appears in Table 5.

Table 5. Summary of osmium dithiocarbamate structures established by X-ray crystallography. See Table 1, Figure 2, and the Abbreviations section.

\begin{tabular}{|c|c|c|c|c|}
\hline Crystal & Formulation & REFCODE & Donor Set & Ref. \\
\hline 157 & $\mathrm{Os}\left(\mathbf{L}^{8}\right)_{2}(\mathrm{CO})_{2}$ & KAZHIE & $\mathrm{C}_{2} \mathrm{~S}_{4}$ & [130] \\
\hline 158 & $\left(\mathrm{PPh}_{3}\right)_{2} \mathrm{Os}\left(\mathrm{SiCl}_{3}\right)\left(\mathbf{L}^{8}\right)(\mathrm{CO}), 2\left(\mathrm{CH}_{2} \mathrm{Cl}_{2}\right)$ & NEKGOA & $\mathrm{CP}_{2} \mathrm{~S}_{2} \mathrm{Si}$ & [125] \\
\hline 159 & $\left(\mathrm{PPh}_{3}\right)_{2} \mathrm{Os}\left(\mathrm{SiMeCl}_{2}\right)\left(\mathrm{L}^{8}\right)(\mathrm{CO}), 2\left(\mathrm{CH}_{2} \mathrm{Cl}_{2}\right)$ & NEKGIU & $\mathrm{CP}_{2} \mathrm{~S}_{2} \mathrm{Si}$ & [125] \\
\hline 160 & $\left(\mathrm{PPh}_{3}\right)_{2} \mathrm{Os}\left(\mathrm{SiMe}_{2} \mathrm{Cl}\right)\left(\mathrm{L}^{8}\right)(\mathrm{CO}), \mathrm{CH}_{2} \mathrm{Cl}_{2}$ & FIXRAG & $\mathrm{CP}_{2} \mathrm{~S}_{2} \mathrm{Si}$ & [126] \\
\hline 161 & $\left(\mathrm{PPh}_{3}\right)_{2} \mathrm{Os}\left(\mathrm{SiMe}_{2} \mathrm{OH}\right)\left(\mathrm{L}^{8}\right)(\mathrm{CO}), x\left(\mathrm{CH}_{3}\left(\mathrm{CH}_{2}\right)_{5} \mathrm{CH}_{3}\right)$ & FIXREK & $\mathrm{CP}_{2} \mathrm{~S}_{2} \mathrm{Si}$ & [126] \\
\hline 162 & $\left(\mathrm{PPh}_{3}\right)_{2} \mathrm{Os}\left(\mathrm{SiMe}_{2} \mathrm{OSiMe}_{3}\right)\left(\mathbf{L}^{8}\right)(\mathrm{CO})$ & FIXROU & $\mathrm{CP}_{2} \mathrm{~S}_{2} \mathrm{Si}$ & [126] \\
\hline 163 & $\left(\mathrm{PPh}_{3}\right)_{2} \mathrm{Os}\left(\mathrm{SiOH}_{3}\right)\left(\mathrm{L}^{8}\right)(\mathrm{CO})$ & FIXRIO & $\mathrm{CP}_{2} \mathrm{~S}_{2} \mathrm{Si}$ & [126] \\
\hline 164 & $\left(\mathrm{PPh}_{3}\right)_{2} \mathrm{Os}\left(\mathrm{SnCl}_{3}\right)\left(\mathrm{L}^{8}\right)(\mathrm{CO}), \mathrm{CHCl}_{3}$ & QEWPAK & $\mathrm{CP}_{2} \mathrm{~S}_{2} \mathrm{Sn}$ & [127] \\
\hline 165 & $\left(\mathrm{PPh}_{3}\right)_{2} \mathrm{Os}\left(\mathrm{SnF}_{3}\right)\left(\mathrm{L}^{8}\right)(\mathrm{CO}), 2\left(\mathrm{CH}_{2} \mathrm{Cl}_{2}\right)$ & QEWPEO & $\mathrm{CP}_{2} \mathrm{~S}_{2} \mathrm{Sn}$ & [127] \\
\hline 166 & $\left(\mathrm{PPh}_{3}\right)_{2} \mathrm{Os}\left(\mathrm{SnH}_{3}\right)\left(\mathrm{L}^{8}\right)(\mathrm{CO}), \mathrm{C}_{6} \mathrm{H}_{6}$ & QEWNUC & $\mathrm{CP}_{2} \mathrm{~S}_{2} \mathrm{Sn}$ & [127] \\
\hline 167 & $\left(\mathrm{PPh}_{3}\right)_{2} \mathrm{Os}\left(\mathrm{SnHMe}_{2}\right)\left(\mathbf{L}^{8}\right)(\mathrm{CO}), \mathrm{C}_{6} \mathrm{H}_{6}$ & QEWPUE & $\mathrm{CP}_{2} \mathrm{~S}_{2} \mathrm{Sn}$ & [127] \\
\hline 168 & $\left(\mathrm{PPh}_{3}\right)_{2} \mathrm{Os}\left(\mathrm{SnMeF}_{2}\right)\left(\mathrm{L}^{8}\right)(\mathrm{CO}), \mathrm{CH}_{2} \mathrm{Cl}_{2}$ & QEWPOY & $\mathrm{CP}_{2} \mathrm{~S}_{2} \mathrm{Sn}$ & [127] \\
\hline 169 & $\left(\mathrm{PPh}_{3}\right)_{2} \mathrm{Os}\left(\mathrm{SnMe}_{2} \mathrm{Cl}\right)\left(\mathrm{L}^{8}\right)(\mathrm{CO}), \mathrm{C}_{6} \mathrm{H}_{6}$ & TAXYOH & $\mathrm{CP}_{2} \mathrm{~S}_{2} \mathrm{Sn}$ & [128] \\
\hline 170 & $\left(\mathrm{PPh}_{3}\right)_{2} \mathrm{Os}\left(\mathrm{SnMe}_{2} \mathrm{SnPh}_{3}\right)\left(\mathrm{L}^{8}\right)(\mathrm{CO}), 2\left(\mathrm{CH}_{2} \mathrm{Cl}_{2}\right)$ & PENLIE & $\mathrm{CP}_{2} \mathrm{~S}_{2} \mathrm{Sn}$ & [129] \\
\hline
\end{tabular}


Table 5. Cont.

\begin{tabular}{|c|c|c|c|c|}
\hline Crystal & Formulation & REFCODE & Donor Set & Ref. \\
\hline 171 & $\left(\mathrm{PPh}_{3}\right) \mathrm{Os}\left(\mathrm{SnMe}_{2} \mathrm{C}_{6} \mathrm{H}_{4} \mathrm{PPh}_{2}\right)\left(\mathrm{L}^{8}\right)(\mathrm{CO})$ & TAXYUN & $\mathrm{CP}_{2} \mathrm{~S}_{2} \mathrm{Sn}$ & [128] \\
\hline 172 & $\left(\mathrm{PPh}_{3}\right) \mathrm{Os}\left(\mathrm{SnMeClC}_{6} \mathrm{H}_{4} \mathrm{PPh}_{2}\right)\left(\mathrm{L}^{8}\right)(\mathrm{CO})$ & TAXZAU & $\mathrm{CP}_{2} \mathrm{~S}_{2} \mathrm{Sn}$ & [128] \\
\hline 173 & $\mathrm{Os}_{3}\left(\mathbf{L}^{8}\right)_{2}(\mathrm{CO})_{10}$ & KUJFED & $\mathrm{C}_{3} \mathrm{OsS}_{2}$ & [131] \\
\hline 174 & {$\left[\mathrm{Os}_{4}\left(\mathrm{~L}^{\mathbf{8}}\right)_{2}(\mathrm{CO})_{12}\left(\mathrm{CO}_{2}\right)(\mathrm{S})\right], 2\left(\mathrm{CH}_{2} \mathrm{Cl}_{2}\right)$} & KUJFIH & $\mathrm{C}_{3} \mathrm{OS}_{2}, \mathrm{C}_{3} \mathrm{~S}_{3}$ & [131] \\
\hline 175 & $\mathrm{Os}_{3}(\mathrm{H})\left(\mathbf{L}^{8}\right)(\mathrm{CO})_{9}$ & KUJFUT & $\mathrm{C}_{3} \mathrm{Os}_{2} \mathrm{~S} / \mathrm{C}_{3} \mathrm{HOs}_{2} \mathrm{~S}$ & [131] \\
\hline 176 & $\mathrm{Os}_{3}(\mathrm{H})\left(\mathrm{L}^{24}\right)(\mathrm{CO})_{9}$ & SATGUQ & $\mathrm{C}_{3} \mathrm{Os}_{2} \mathrm{~S} / \mathrm{C}_{3} \mathrm{HOs}_{2} \mathrm{~S}$ & [132] \\
\hline
\end{tabular}

Of the mononuclear molecules, 157 [130] is exceptional for two reasons: it is the only molecule with two dithiocarbamate ligands and does not feature a phosphane ligand. As seen from Figure 10a, the osmium atom is coordinated by two chelating dithiocarbamate ligands and two carbonyls, the latter being mutually cis. The common feature of $\mathbf{1 5 8}$ [125], $\mathbf{1 5 9}$ [125], $\mathbf{1 6 0}$ [126], $\mathbf{1 6 1}$ [126], $\mathbf{1 6 2}$ [126], and $\mathbf{1 6 3}$ [126], is a $\mathrm{CP}_{2} \mathrm{~S}_{2} \mathrm{Si}$ donor set. As exemplified for $\mathbf{1 5 8}$ in Figure 10b, the osmium atom is coordinated by two mutually trans phosphane ligands, a carbonyl, a trisubstituted silicon anion and a chelating dithiocarbamate anion. The molecules in 164 [127], 165 [127], 166 [127], 167 [127], 168 [127], 169 [128], 170 [129], 171 [128], and 172 [128] adopt similar coordination geometries whereby the silicon atoms of the previous series are substituted for tin atoms. An exemplar is 170, shown in Figure 10c, which is notable for having a dinuclear tin substituent.

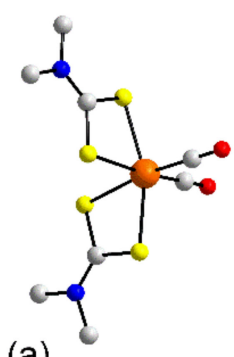

(a)

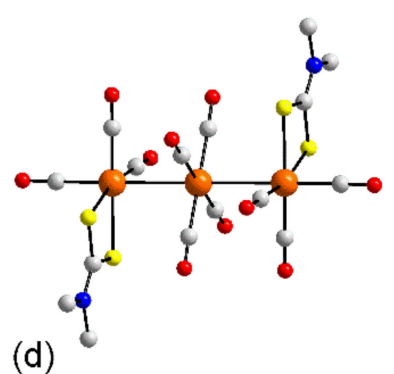

(b)

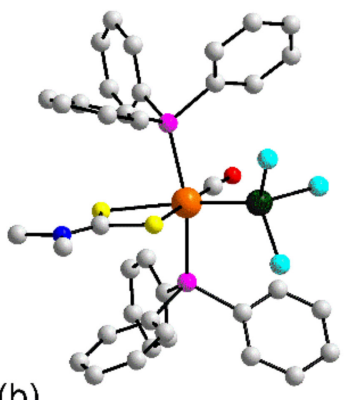

(e)

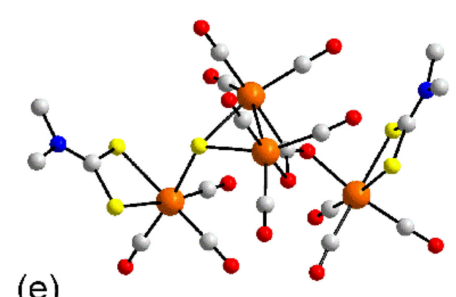

(f)

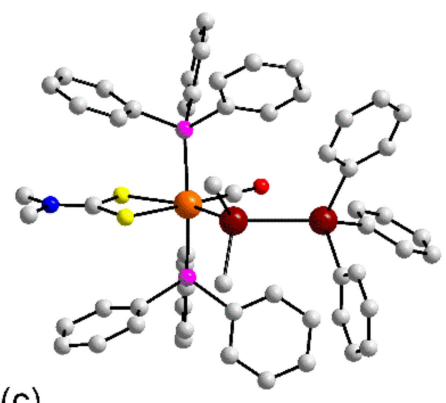

(c)

Figure 10. Molecular structures of osmium complexes: (a) $\mathrm{Os}\left(\mathbf{L}^{8}\right)_{2}(\mathrm{CO})_{2}(\mathbf{1 5 7}),(\mathbf{b})\left(\mathrm{PPh}_{3}\right)_{2} \mathrm{Os}\left(\mathrm{SiCl}_{3}\right)\left(\mathbf{L}^{\mathbf{8}}\right)$ (CO) in the crystal of 158, (c) $\left(\mathrm{PPh}_{3}\right)_{2} \mathrm{Os}\left(\mathrm{SnMe}_{2} \mathrm{SnPh}_{3}\right)\left(\mathbf{L}^{\mathbf{8}}\right)(\mathrm{CO})$ in $\mathbf{1 7 0}$, (d) $\mathrm{Os}_{3}\left(\mathbf{L}^{\mathbf{8}}\right)_{2}(\mathrm{CO})_{10}(\mathbf{1 7 3})$, $(\mathbf{e}) \mathrm{Os}_{4}\left(\mathbf{L}^{8}\right)_{2}(\mathrm{CO})_{12}\left(\mathrm{CO}_{2}\right)(\mathrm{S})$ in $\mathbf{1 7 4}$, and $(\mathbf{f}) \mathrm{Os}_{3}(\mathrm{H})\left(\mathbf{L}^{24}\right)(\mathrm{CO})_{9}(\mathbf{1 7 6})$.

The remaining crystals feature cluster molecules having three, i.e., 173 [131], 175 [131], and 176 [131] or four, 174 [131] osmium atoms. In 173, Figure 10d, the terminal osmium atoms in a chain of three osmium atoms are each chelated by a dithiocarbamate ligand, with further coordination provided by three carbonyl ligands and the octahedral geometry completed by the central osmium atom; the latter features a $\mathrm{C}_{4} \mathrm{Os}_{2}$ donor set. In the tetranuclear cluster of 174, Figure 10e, again each terminal osmium atom is chelated by a dithiocarbamate ligand. The coordination geometry for one of the osmium atoms is 
based on a $\mathrm{C}_{3} \mathrm{~S}_{3}$ donor set as three of the osmium atoms are $\mu_{3}$-bridged by a sulfide-S atom while the second terminal osmium atom has a $\mathrm{C}_{3} \mathrm{OS}_{2}$ coordination geometry as the $\mu_{3}$-carbon dioxide atom employs all three atoms in coordination to three different osmium atoms. Finally, the osmium atoms of $\mathbf{1 7 5}$ and $\mathbf{1 7 6}$ are arranged in a triangle. As shown for $\mathbf{1 7 6}$ in Figure 10f, the dithiocarbamate ligand forms bonds with each of the osmium atoms, forming a bond to one ring osmium atom and at the same time connects to the other two osmium atoms via the second sulfur atom. The osmium atoms that are bridged by the $\mu_{2}$-sulfur atom are also bridged by a $\mu_{2}$-hydride atom. Thus, there are two distinct coordination geometries, i.e., $\mathrm{C}_{3} \mathrm{Os}_{2} \mathrm{~S}$ and $\mathrm{C}_{3} \mathrm{HOs}_{2} \mathrm{~S}$.

\subsubsection{Iridium}

Iridium dithiocarbamates are commonly seen as the organometallic complexes as presented in Table 6 . The iridium complexes are characterized by coordination number six within a distorted octahedral geometry. As with previous precious group elements, a relatively high degree of commonality is noted in the molecular structures, with structural precedents also in existence. The most presented structural motif among the iridium dithiocarbamates is illustrated in Figure 11a for $\mathbf{1 7 7}$ [133]. The iridium atom is chelated by the dithiocarbamate ligand, N,C-chelated by each of two 2-(2-pyridyl)phenyl anions, leading to a $\mathrm{C}_{2} \mathrm{~N}_{2} \mathrm{~S}_{2}$ donor set, with trans-nitrogen atoms, which defines an octahedral geometry. Similar structures but with varying substitution patterns in the dithiocarbamate and N,C-chelating ligands are found in the crystals of $\mathbf{1 7 8}$ [134], 179 [135], 180 [136], 181 [136], 182 [136], 183 [137], 184 [137], 185 [137], 186 [27], 187 [138], 188 [139], 189 [139], 190 [139], 191 [140], 192 [138], 193 [140], 194 [141], 195 [142], 196 [143,144], 197 [145], and $198[146,147]$.

Table 6. Summary of iridium dithiocarbamate structures established by X-ray crystallography. See Table 1, Figure 2, and the Abbreviations section.

\begin{tabular}{|c|c|c|c|c|}
\hline Crystal & Formulation & REFCODE & Donor Set & Ref. \\
\hline 177 & $\operatorname{Ir}(\text { ppy })_{2}\left(\mathbf{L}^{24}\right)$ & WIHPUZ & $\mathrm{C}_{2} \mathrm{~N}_{2} \mathrm{~S}_{2}$ & [133] \\
\hline 178 & $\operatorname{Ir}(\text { buppy })_{2}\left(\mathrm{~L}^{24}\right)$ & MADKAE & $\mathrm{C}_{2} \mathrm{~N}_{2} \mathrm{~S}_{2}$ & [134] \\
\hline 179 & $\operatorname{Ir}(\mathrm{pba})_{2}\left(\mathbf{L}^{24}\right)$ & QOFTEN & $\mathrm{C}_{2} \mathrm{~N}_{2} \mathrm{~S}_{2}$ & [135] \\
\hline 180 & $\operatorname{Ir}(\operatorname{tfmppy})_{2}\left(\mathbf{L}^{29}\right)$ & FODDUA & $\mathrm{C}_{2} \mathrm{~N}_{2} \mathrm{~S}_{2}$ & [136] \\
\hline 181 & $\operatorname{Ir}(\text { btfmppy })_{2}\left(\mathbf{L}^{29}\right)$ & FODDOU & $\mathrm{C}_{2} \mathrm{~N}_{2} \mathrm{~S}_{2}$ & [136] \\
\hline 182 & $\operatorname{Ir}(\operatorname{tfmbpy})_{2}\left(\mathbf{L}^{29}\right)$ & FODFAI & $\mathrm{C}_{2} \mathrm{~N}_{2} \mathrm{~S}_{2}$ & [136] \\
\hline 183 & $\operatorname{Ir}(\operatorname{tfmpiq})_{2}\left(\mathbf{L}^{29}\right)$ & HOFCUD & $\mathrm{C}_{2} \mathrm{~N}_{2} \mathrm{~S}_{2}$ & [137] \\
\hline 184 & $\operatorname{Ir}(\operatorname{tfmpiq})_{2}\left(\mathrm{~L}^{46}\right)$, unknown solvate & HOFDAK & $\mathrm{C}_{2} \mathrm{~N}_{2} \mathrm{~S}_{2}$ & [137] \\
\hline 185 & $\operatorname{Ir}(\operatorname{tfmpiq})_{2}\left(\mathbf{L}^{83}\right)$, unknown solvate & HOFDEO & $\mathrm{C}_{2} \mathrm{~N}_{2} \mathrm{~S}_{2}$ & [137] \\
\hline 186 & $\operatorname{Ir}(\mathrm{pqz})_{2}\left(\mathbf{L}^{\mathbf{8 2}}\right)$ & ZUQLUU & $\mathrm{C}_{2} \mathrm{~N}_{2} \mathrm{~S}_{2}$ & [27] \\
\hline 187 & $\operatorname{Ir}(\mathrm{tfmpqz})_{2}\left(\mathrm{~L}^{29}\right)$ & LIYWUO & $\mathrm{C}_{2} \mathrm{~N}_{2} \mathrm{~S}_{2}$ & [138] \\
\hline 188 & $\operatorname{Ir}(\operatorname{tfmpqz})_{2}\left(\mathrm{~L}^{46}\right)$ & CUMGUP & $\mathrm{C}_{2} \mathrm{~N}_{2} \mathrm{~S}_{2}$ & [139] \\
\hline 189 & $\operatorname{Ir}(\mathrm{tfmpqz})_{2}\left(\mathrm{~L}^{48}\right)$ & CUMGOJ & $\mathrm{C}_{2} \mathrm{~N}_{2} \mathrm{~S}_{2}$ & [139] \\
\hline 190 & $\operatorname{Ir}(\operatorname{tfmpqz})_{2}\left(\mathrm{~L}^{49}\right)$ & CUMHOK & $\mathrm{C}_{2} \mathrm{~N}_{2} \mathrm{~S}_{2}$ & [139] \\
\hline 191 & $\operatorname{Ir}(\operatorname{tfmpqz})_{2}\left(\mathbf{L}^{50}\right)$ & VIWDAJ & $\mathrm{C}_{2} \mathrm{~N}_{2} \mathrm{~S}_{2}$ & [140] \\
\hline 192 & $\operatorname{Ir}(\mathrm{tfmpqz})_{2}\left(\mathbf{L}^{82}\right)$ & LIYWOI & $\mathrm{C}_{2} \mathrm{~N}_{2} \mathrm{~S}_{2}$ & [138] \\
\hline 193 & $\operatorname{Ir}(\operatorname{tfmpqz})_{2}\left(\mathbf{L}^{83}\right)$ & VIWCUC & $\mathrm{C}_{2} \mathrm{~N}_{2} \mathrm{~S}_{2}$ & [140] \\
\hline 194 & $\operatorname{Ir}(\mathrm{dpp})_{2}\left(\mathrm{~L}^{24}\right), \mathrm{CH}_{2} \mathrm{Cl}_{2}$, unknown solvate & IQAFEN & $\mathrm{C}_{2} \mathrm{~N}_{2} \mathrm{~S}_{2}$ & [141] \\
\hline 195 & $\operatorname{Ir}(\mathrm{dpci})_{2}\left(\mathrm{~L}^{82}\right), \mathrm{CH}_{2} \mathrm{Cl}_{2}, 1.5\left(\mathrm{H}_{2} \mathrm{O}\right)$ & VITHEN & $\mathrm{C}_{2} \mathrm{~N}_{2} \mathrm{~S}_{2}$ & [142] \\
\hline 196 & $\operatorname{Ir}(\mathrm{pbt})_{2}\left(\mathrm{~L}^{24}\right)$ & IXOJEM * & $\mathrm{C}_{2} \mathrm{~N}_{2} \mathrm{~S}_{2}$ & {$[143,144]$} \\
\hline 197 & $\operatorname{Ir}(\mathrm{ptbt})_{2}\left(\mathrm{~L}^{24}\right)$ & WOTQUU & $\mathrm{C}_{2} \mathrm{~N}_{2} \mathrm{~S}_{2}$ & [145] \\
\hline 198 & $\operatorname{Ir}\left(\mathrm{X}^{4}\right)_{2}\left(\mathbf{L}^{24}\right), 0.5\left(\mathrm{H}_{2} \mathrm{O}\right)$ & WEKROU * & $\mathrm{C}_{2} \mathrm{~N}_{2} \mathrm{~S}_{2}$ & {$[146,147]$} \\
\hline 199 & $\left(\mathrm{PPh}_{3}\right)_{2} \operatorname{Ir}\left(\mathrm{C}_{5} \mathrm{H}_{5} \mathrm{SMe}\right)\left(\mathrm{L}^{24}\right)$ & ANOKAQ & $\mathrm{C}_{2} \mathrm{P}_{2} \mathrm{~S}_{2}$ & [148] \\
\hline 200 & {$\left[\left(\mathrm{PPh}_{3}\right)_{2} \operatorname{Ir}\left(\mathrm{C}_{5} \mathrm{H}_{4} \mathrm{SMe}\right)\left(\mathbf{L}^{24}\right)\right] \mathrm{PF}_{6}$} & ANOJUJ & $\mathrm{C}_{2} \mathrm{P}_{2} \mathrm{~S}_{2}$ & [148] \\
\hline 201 & {$\left[\left(\mathrm{PPh}_{3}\right)_{2} \mathrm{Ir}\left(\mathrm{C}_{5} \mathrm{H}_{3} \mathrm{Me}-3 \mathrm{SMe}\right)\left(\mathrm{L}^{24}\right)\right] \mathrm{PF}_{6}, \mathrm{ClCH}_{2} \mathrm{CH}_{2} \mathrm{Cl}$} & ANOKEU & $\mathrm{C}_{2} \mathrm{P}_{2} \mathrm{~S}_{2}$ & [148] \\
\hline 202 & {$\left[\left(\mathrm{PPh}_{3}\right)_{2} \mathrm{Ir}\left(\mathrm{C}_{5} \mathrm{H}_{3} \mathrm{OEt}-3 \mathrm{SMe}\right)\left(\mathbf{L}^{24}\right)\right] \mathrm{PF}_{6}$} & ANOKIY & $\mathrm{C}_{2} \mathrm{P}_{2} \mathrm{~S}_{2}$ & [148] \\
\hline 203 & {$\left[\mathrm{Cp}^{*} \operatorname{Ir}\left(\mathrm{L}^{24}\right)(\mathrm{CO})\right] \mathrm{CF}_{3} \mathrm{SO}_{3}$} & GEVGUK & $\mathrm{C}_{4} \mathrm{~S}_{2}$ & [149] \\
\hline
\end{tabular}


Table 6. Cont.

\begin{tabular}{|c|c|c|c|c|}
\hline Crystal & Formulation & REFCODE & Donor Set & Ref. \\
\hline 204 & {$\left[\mathrm{Cp} * \operatorname{Ir}\left(\mathbf{L}^{24}\right)\right]_{2}\left[\mathrm{ClO}_{4}\right]_{2}$} & WIHNIL & $\mathrm{C}_{3} \mathrm{~S}_{3}$ & [150] \\
\hline 205 & $\mathrm{Cp} \mathrm{p}^{*} \operatorname{Ir}(\mathrm{mtz})\left(\mathbf{L}^{8}\right)$, 5-methyltetrazole & NOJPUY & $\mathrm{C}_{3} \mathrm{NS}_{2}$ & [151] \\
\hline 206 & {$\left[\left\{\mathrm{C} \mathrm{p}^{*} \operatorname{Ir}\left(\mathrm{L}^{8}\right)\right\}_{2}(\mathrm{mtz})\right] \mathrm{PF}_{6}$} & NOJQAF & $\mathrm{C}_{3} \mathrm{NS}_{2}$ & [151] \\
\hline 207 & $\mathrm{Cp}{ }^{*} \operatorname{Ir}(\mathrm{SH})\left(\mathrm{PMe}_{3}\right)\left(\mathrm{L}^{5}\right), \mathrm{CH}_{2} \mathrm{Cl}_{2}$ & SIKPUY & $\mathrm{C}_{3} \mathrm{PS}_{2}$ & [152] \\
\hline 208 & $\mathrm{Cp} \mathrm{p}^{*} \operatorname{Ir}(\mathrm{H})\left(\mathrm{PMe}_{3}\right)\left(\mathrm{L}^{5}\right)$ & SIKQEJ & $\mathrm{C}_{3} \mathrm{HPS}$ & [152] \\
\hline 209 & {$\left[\mathrm{C} \mathrm{p}^{*} \operatorname{Ir}\left(\mathrm{PPh}_{3}\right)\left(\mathrm{L}^{24}\right)\right]_{3}\left[\mathrm{Cp} \mathrm{p}^{*} \operatorname{Ir}\left(\mathrm{PPh}_{3}\right)\left(\mathrm{L}^{25}\right)\left(\mathrm{NCH}_{3}\right)\right][\mathrm{I}]_{4}$} & OYUJEY & $\mathrm{C}_{3} \mathrm{NPS}$ & [153] \\
\hline
\end{tabular}

* CSD depositions are available for repeated measurements.

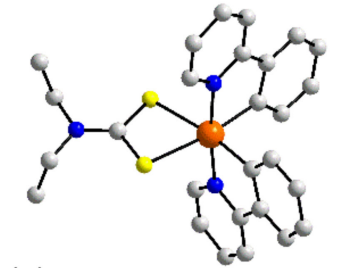

(a)

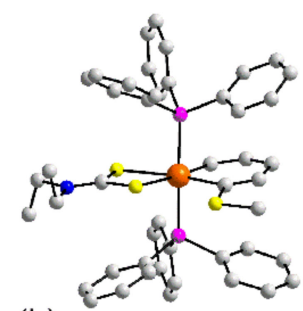

(b)

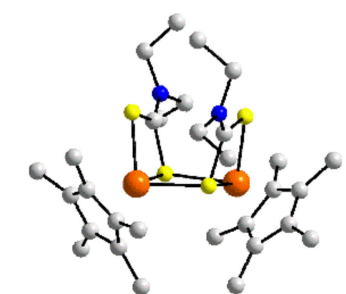

(c)

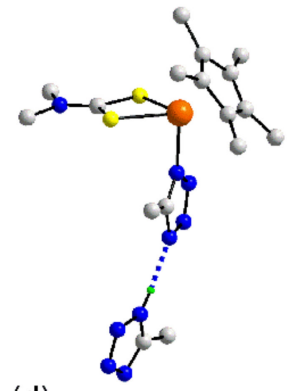

(d)

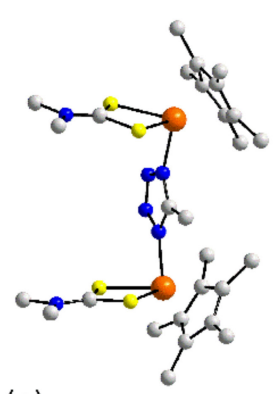

(e)

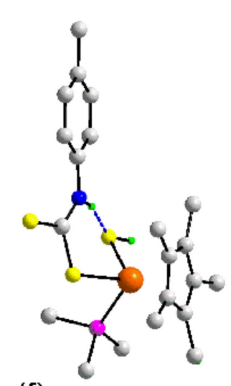

(f)

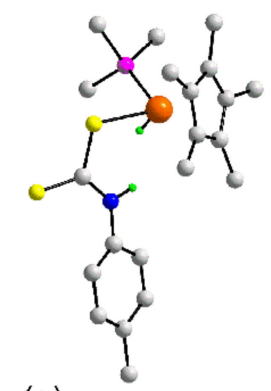

(g)

Figure 11. Molecular structures of iridium dithiocarbamate complexes: (a) $\operatorname{Ir}(\mathrm{ppy})_{2}\left(\mathbf{L}^{\mathbf{2 4}}\right)(\mathbf{1 7 7})$, (b) $\left(\mathrm{PPh}_{3}\right)_{2} \operatorname{Ir}\left(\mathrm{C}_{5} \mathrm{H}_{5} \mathrm{SMe}\right)\left(\mathbf{L}^{24}\right)(\mathbf{1 9 9}),(\mathbf{c})\left[\mathrm{C} \mathrm{p}^{*} \operatorname{Ir}\left(\mathbf{L}^{24}\right)\right]_{2}{ }^{2+}$ in the crystal of 204, (d) $\mathrm{Cp} \mathrm{p}^{*} \operatorname{Ir}(\mathrm{mtz})\left(\mathbf{L}^{8}\right)$ in 205, (e) $\left[\left\{\mathrm{Cp} \mathrm{P}^{*} \operatorname{Ir}\left(\mathbf{L}^{8}\right)\right\}_{2}(\mathrm{mtz})\right]^{+}$in 206, (f) $\mathrm{Cp} \mathrm{p}^{*} \operatorname{Ir}\left(\mathrm{PMe}_{3}\right)\left(\mathrm{L}^{5}\right)(\mathrm{SH})$ in 207, and $(\mathrm{g}) \mathrm{Cp}{ }^{*} \operatorname{Ir}(\mathrm{H})\left(\mathrm{PMe}_{3}\right)\left(\mathbf{L}^{5}\right)(\mathbf{2 0 8})$. In (d) and (f), the blue dashed line represents $\mathrm{N}-\mathrm{H} \cdots \mathrm{N}$ and $\mathrm{N}-\mathrm{H} \cdots \mathrm{S}$ hydrogen bonds, respectively.

In crystals of 199 [148], 200 [148], 201 [148], and 202 [148] a $\mathrm{C}_{2} \mathrm{P}_{2} \mathrm{~S}_{2}$ donor set is featured. In neutral 199, Figure 11b, the iridium center is chelated by dithiocarbamate and C,C-chelating (1-(methylsulfanyl)penta-1,4-diene-1,5-diyl ligands with the phosphane molecules occupying mutually trans positions. The complex molecules in 200-202 are cationic and adopt the same structural motif. The remaining iridium dithiocarbamate structures feature $C \mathrm{p}^{*}$ ligands. The cation in 203 [149] has a geometry reminiscent of the ruthenium analog shown in Figure 5c. A new structural motif is noted for the dication in 204 [150]. Each dithiocarbamate ligand chelates one iridium atom and uses one of the sulfur atoms to bridge the adjacent iridium atom. While not crystallographically imposed, the molecule has approximately 2-fold symmetry so the dithiocarbamate ligands lie to the same side of the central $\operatorname{Ir}_{2} S_{2}$ rectangle. In 205 [151], the carbonyl of 203 has been replaced by a 5-methyltetrazolate to give a neutral complex. As noted from Figure 11d, the anion is hydrogen bonded to 5-methyltetrazole in the crystal. In the cation of 206 [151], the hydrogen bonded molecule of $\mathbf{2 0 5}$ is replaced by a $\left[\mathrm{Cp}^{*} \operatorname{Ir}\left(\mathbf{L}^{8}\right)\right]$ to give the dinuclear molecule shown in Figure 11e. The common feature of 207 [152] and 208 [152] is the coordination by a monodentate dithiocarbamate ligand, rather unusual in the context of the present survey. In 207, Figure 11f, the iridium atom has a $C_{3} P_{2}$ donor set with the second sulfur atom derived from a hydrosulfide anion. An intramolecular hydrogen bond is noted between the dithiocarbamate-N-H $\cdots$ S(hydrosulfide) atoms. In 208, another rare example 
where the dithiocarbamate anion is derived from a primary amine, the hydrosulfide anion of 207 has been replaced by a hydride, Figure 11g. The last iridium structure comprises three independent $\left[\mathrm{Cp}^{*} \operatorname{Ir}\left(\mathrm{PPh}_{3}\right)\left(\mathrm{L}^{25}\right)\left(\mathrm{NCH}_{3}\right)\right]$ cation, and four iodide counterions in the crystal of 209 [153]. The dithiocarbamate cation adopts the structural motif illustrated in Figure 5a.

\subsubsection{Platinum}

As for the palladium dithiocarbamates, binary structures predominate among the platinum dithiocarbamates which have been characterized by X-ray crystallography, see Table 7 for a listing. Thus, square planar geometries based on $S_{4}$ donor sets provided by two chelating dithiocarbamates ligands are found in 210 [73], 211 [73], 212 [75,76], $2 \mathbf{2 1 3}$ [80], 214 [154], 215 [155], 216 [156], 217 [84], 218 [155,157], 219 [157], 220 [158], 221 [159], 222 [160], 223 [161], 224 [86], 225 [162], 226 [163], and 227 [164]. An exemplar molecule is shown for 222 in Figure 12a. This is particularly notable for having additional functionality on the dithiocarbamate ligands giving rise to the possibility of further complexation to other metals. The only binary dithiocarbamate complex that does not conform to the common structural motif is that of 228 [165]. As shown in Figure 12b, the dithiocarbamate ligand carries a pendant pyrazolyl ring and in the case of one dithiocarbamate ligand, a $\mathrm{S}, \mathrm{N}$-chelating mode occurs rather than the normal S,S-chelating mode so the resulting donor set is $\mathrm{NS}_{3}$. As alluded to above, the pendent carboxylic acid residues in $\mathbf{2 2 8}$ can be employed in further coordination to generate higher-dimensional aggregation patterns. This possibility has been realized in four crystals, namely 229 [161], 230 [161], 231 [161], and 232 [161]. A one-dimensional coordination polymer results as each $\mathbf{L}^{\mathbf{1 7}}$ ligand is deprotonated at one of the carboxylic acid residues and complexation occurs with one equivalent of $\mathrm{Zn}\left(\mathrm{OH}_{2}\right)_{4}$. The polymer 229, as in Figure 12c, representative of those in 230 and 231, has a step-like topology. As for 229-231, a square planar geometry based on the $\mathrm{S}_{4}$ donor set is evident for the platinum atom in 232. Here, both carboxylic acid residues of each $\mathbf{L}^{\mathbf{5 2}}$ anion are deprotonated, giving $\mathbf{L}^{\mathbf{5 1}}$. When complexed with two equivalents of $\mathrm{Zn}\left(\mathrm{OH}_{2}\right)_{4}$, a two-dimensional grid with a flat topology ensues, as illustrated in the plan view of Figure 12d. In all four coordination polymers, the carboxylate ligand is functioning in the monodentate mode.

Table 7. Summary of platinum dithiocarbamate structures established by X-ray crystallography. See Table 1, Figure 2, and the Abbreviations section.

\begin{tabular}{|c|c|c|c|c|}
\hline Crystal & Formulation & REFCODE & Donor Set & Ref. \\
\hline 210 & $\operatorname{Pt}\left(\mathbf{L}^{4}\right)_{2}$ & YIRSOK & $\mathrm{S}_{4}$ & [73] \\
\hline 211 & $\operatorname{Pt}\left(\mathbf{L}^{6}\right)_{2}$ & YIRXEF & $\mathrm{S}_{4}$ & [73] \\
\hline 212 & $\operatorname{Pt}\left(\mathbf{L}^{8}\right)_{2}$ & TEDRIG * & $\mathrm{S}_{4}$ & {$[75,76]$} \\
\hline 213 & $\operatorname{Pt}\left(\mathbf{L}^{16}\right)_{2}$ & FUHXIR & $\mathrm{S}_{4}$ & [80] \\
\hline 214 & $\operatorname{Pt}\left(\mathrm{L}^{27}\right)_{2}$ & TUCMAH & $\mathrm{S}_{4}$ & [154] \\
\hline 215 & $\operatorname{Pt}\left(\mathbf{L}^{29}\right)_{2}$ & LOWJEP & $\mathrm{S}_{4}$ & [155] \\
\hline 216 & {$\left[\mathrm{Pt}\left(\mathbf{L}^{31}\right)_{2}\right], \mathrm{C}_{60}$} & MOKNOR & $\mathrm{S}_{4}$ & [156] \\
\hline 217 & $\operatorname{Pt}\left(\mathbf{L}^{33}\right)_{2}$ & EMOKUO & $\mathrm{S}_{4}$ & [84] \\
\hline 218 & $\operatorname{Pt}\left(\mathrm{L}^{34}\right)_{2}$ & ZOJHUE & $\mathrm{S}_{4}$ & {$[155,157]$} \\
\hline 219 & {$\left[\mathrm{Pt}\left(\mathrm{L}^{39}\right)_{2}\right], \mathrm{C}_{6} \mathrm{H}_{5} \mathrm{CN}$} & ZOJJAM & $\mathrm{S}_{4}$ & [157] \\
\hline 220 & $\mathrm{Pt}\left(\mathrm{L}^{41}\right)_{2}$ & LASPIH & $\mathrm{S}_{4}$ & [158] \\
\hline 221 & $\operatorname{Pt}\left(\mathbf{L}^{45}\right)_{2}$ & UHOGIJ & $\mathrm{S}_{4}$ & [159] \\
\hline 222 & $\operatorname{Pt}\left(\mathbf{L}^{52}\right)_{2}$ & MOHNUU & $\mathrm{S}_{4}$ & [160] \\
\hline 223 & {$\left[\mathrm{Pt}\left(\mathrm{L}^{52}\right)_{2}\right], 6\left(\left(\mathrm{CH}_{3}\right)_{2} \mathrm{SO}\right)$} & GUCQAZ & $\mathrm{S}_{4}$ & [161] \\
\hline 224 & $\operatorname{Pt}\left(\mathbf{L}^{54}\right)_{2}$ & HOMXАK & $\mathrm{S}_{4}$ & [86] \\
\hline 225 & $\operatorname{Pt}\left(\mathbf{L}^{55}\right)_{2}$, unknown solvate & GUMCEZ & $\mathrm{S}_{4}$ & [162] \\
\hline 226 & $\operatorname{Pt}\left(\mathbf{L}^{70}\right)_{2}$ & WIFVUF & $\mathrm{S}_{4}$ & [163] \\
\hline 227 & $\operatorname{Pt}\left(\mathbf{L}^{80}\right)_{2}$ & CAJWOC & $\mathrm{S}_{4}$ & [164] \\
\hline 228 & $\operatorname{Pt}\left(\mathrm{L}^{62}\right)_{2}$ & GEZHEA & $\mathrm{S}_{3} \mathrm{~N}$ & [165] \\
\hline 229 & {$\left[\mathrm{Zn}\left(\mathrm{H}_{2} \mathrm{O}\right)_{4} \mathrm{Pt}\left(\mathrm{L}^{17}\right)_{2}\right]_{\mathrm{n}}, 4\left(\mathrm{H}_{2} \mathrm{O}\right)$} & GUCQED & $\mathrm{S}_{4}$ & [161] \\
\hline 230 & {$\left[\mathrm{Zn}\left(\mathrm{H}_{2} \mathrm{O}\right)_{4} \mathrm{Pt}\left(\mathrm{L}^{58}\right)_{2}\right]_{\mathrm{n}}, 2\left(\mathrm{H}_{2} \mathrm{O}\right)$} & GUCQIH & $\mathrm{S}_{4}$ & [161] \\
\hline
\end{tabular}


Table 7. Cont.

\begin{tabular}{|c|c|c|c|c|}
\hline Crystal & Formulation & REFCODE & Donor Set & Ref. \\
\hline 231 & $\left\{\mathrm{Zn}\left(\mathrm{H}_{2} \mathrm{O}\right)_{3} \mathrm{Pt}\left(\mathbf{L}^{53}\right)_{2}\right]_{n}, 3\left(\mathrm{H}_{2} \mathrm{O}\right)$ & GUCQUT & $\mathrm{S}_{4}$ & [161] \\
\hline 232 & $\left\{\left[\mathrm{Zn}\left(\mathrm{H}_{2} \mathrm{O}\right)_{4}\right]_{2} \mathrm{Pt}\left(\mathbf{L}^{51}\right)_{2}\right\}_{n}, 6\left(\mathrm{H}_{2} \mathrm{O}\right)$ & GUCQONN & $\mathrm{S}_{4}$ & [161] \\
\hline 233 & {$\left[\mathrm{Pt}\left(\mathrm{L}^{57}\right) \mathrm{Cl}\right]_{5}, 2\left(\mathrm{ClCH}_{2} \mathrm{CH}_{2} \mathrm{Cl}\right)$} & HACCAR & $\mathrm{ClS}_{3}$ & [166] \\
\hline 234 & $\left(\mathrm{PPh}_{3}\right) \mathrm{Pt}\left(\eta^{1}-\mathrm{L}^{57}\right)\left(\eta^{2}-\mathrm{L}^{57}\right), 2\left(\mathrm{CH}_{2} \mathrm{Cl}_{2}\right), 0.25\left(\mathrm{CH}_{3}\left(\mathrm{CH}_{2}\right)_{3} \mathrm{CH}_{3}\right)$ & DIFQOA & $\mathrm{PS}_{3}$ & {$[26]$} \\
\hline 235 & $\left\{\left[\left(\mathrm{PEt}_{3}\right)_{2} \mathrm{Pt}_{2}\left(\mathrm{~L}^{74}\right)\right\}\left(\mathrm{PF}_{6}\right)_{2}\right.$, unknown solvate & RUDLAE & $\mathrm{P}_{2} \mathrm{~S}_{2}$ & [101] \\
\hline 236 & $\left\{\left[\left(\mathrm{PPh}_{3}\right)_{2} \mathrm{Pt}\right]_{2}\left(\mathrm{~L}^{74}\right)\right\}\left(\mathrm{PF}_{6}\right)_{2}$ & RUDKUX & $\mathrm{P}_{2} \mathrm{~S}_{2}$ & [101] \\
\hline 237 & $\mathrm{Pt}\left(\mathrm{L}^{57}\right)(\mathrm{Cl})\left(\left(\mathrm{CH}_{3}\right)_{2} \mathrm{SO}\right)$ & NASDAO & $\mathrm{ClS}_{3}$ & [167] \\
\hline 238 & $\left(\mathrm{PEt}_{3}\right) \mathrm{Pt}\left(\mathrm{L}^{24}\right) \mathrm{Cl}$ & TOWNUR & $\mathrm{ClPS}_{2}$ & [168] \\
\hline 239 & $\left(\mathrm{PPh}_{3}\right) \operatorname{Pt}\left(\mathrm{L}^{57}\right) \mathrm{Cl}, 0.5\left(\mathrm{CH}_{2} \mathrm{Cl}_{2}\right)$ & HACBUK & $\mathrm{ClPS}_{2}$ & [166] \\
\hline 240 & {$\left[\mathrm{P}\left(\mathrm{C}_{6} \mathrm{H}_{4} \mathrm{Cl}-\mathrm{p}\right)_{3}\right] \mathrm{Pt}\left(\mathbf{L}^{73}\right) \mathrm{Cl}$} & AZIDAQ & $\mathrm{ClPS}_{2}$ & [169] \\
\hline 241 & {$\left[\mathrm{P}\left(\mathrm{C}_{6} \mathrm{H}_{4} \mathrm{~F}-\mathrm{p}\right)_{3}\right] \mathrm{Pt}\left(\mathrm{L}^{73}\right) \mathrm{Cl}$} & AZIDEU & $\mathrm{ClPS}_{2}$ & [169] \\
\hline 242 & {$\left[\mathrm{P}\left(\mathrm{C}_{6} \mathrm{H}_{4} \mathrm{~F}-\mathrm{p}\right)_{3}\right] \mathrm{Pt}\left(\mathbf{L}^{78}\right) \mathrm{Cl}$} & AZICUJ & $\mathrm{ClPS}_{2}$ & [169] \\
\hline 243 & {$\left[\mathrm{P}(\mathrm{p} \text {-tolyl })_{3}\right] \mathrm{Pt}\left(\mathrm{L}^{76}\right) \mathrm{Cl}$} & RIZNEW & $\mathrm{ClPS}_{2}$ & [170] \\
\hline 244 & $\mathrm{Pt}(\mathrm{bmi})\left(\mathrm{L}^{24}\right) \mathrm{I}$ & AHADAR & $\mathrm{CIS}_{2}$ & [171] \\
\hline 245 & $\operatorname{Pt}(\mathrm{bzq})\left(\mathrm{L}^{8}\right)$ & EQONOO & $\mathrm{CNS}_{2}$ & [172] \\
\hline 246 & $\operatorname{Pt}(\mathrm{bzq})\left(\mathrm{L}^{57}\right)$ & EQONII & $\mathrm{CNS}_{2}$ & [172] \\
\hline 247 & $\operatorname{Pt}\left(X^{5}\right)\left(L^{24}\right)$ & PUPHUF & $\mathrm{CNS}_{2}$ & [173] \\
\hline 248 & {$\left[\mathrm{Pt}\left(\mathbf{L}^{24}\right)\right]_{2}(\mathrm{bpy}), \mathrm{CH}_{2} \mathrm{Cl}_{2}$} & RIZBUA & $\mathrm{CNS}_{2}$ & [174] \\
\hline 249 & $\left(\mathrm{PEt}_{3}\right) \operatorname{Pt}\left(\mathrm{L}^{24}\right)\left(\mathrm{CH}_{2}(\mathrm{iPr})\right)$ & HUWGIR & $\mathrm{CPS}_{2}$ & [175] \\
\hline 250 & $\mathrm{Pt}\left(\mathrm{PPh}_{3}\right)\left(\mathrm{L}^{24}\right) \mathrm{C} \equiv \mathrm{W}(\mathrm{CO})_{2}\left(\mathrm{Tp}^{*}\right)$ & JUQRAR & $\mathrm{CPS}_{2}$ & [176] \\
\hline 251 & $\mathrm{Pt}(\mathrm{bzq})\left(\mathrm{L}^{8}\right)\left(\mathrm{PPh}_{2}\right)_{2} \mathrm{Pt}\left(\mathrm{C}_{6} \mathrm{~F}_{5}\right)_{2}, 2\left(\mathrm{CH}_{2} \mathrm{Cl}_{2}\right)$ & QUFXAT & $\mathrm{CNP}_{2} \mathrm{~S}_{2}$ & [177] \\
\hline 252 & $\left\{\mathrm{Ag}\left[\mathrm{Pt}(\mathrm{bzq})\left(\mathrm{L}^{57}\right)\right]_{2}\right\}_{\mathrm{n}}, \mathrm{n}\left(\mathrm{ClO}_{4}\right), \mathrm{n}\left(\mathrm{CH}_{3} \mathrm{CN}\right)$ & EQOPEG & $\mathrm{AgCNS}_{2}$ & [172] \\
\hline
\end{tabular}

* CSD depositions are available for repeated measurements.

An unusual pentanuclear structure is found in the crystal of 233 [166]. As indicated in Figure 13a, each dithiocarbamate ligand chelates a platinum atom and simultaneously links to a second platinum via one sulfur atom only. The square planar geometry is completed by a chloride atom. The addition of a phosphane ligand to a binary dithiocarbamate results in one of the dithiocarbamate ligands coordinating in a monodentate mode, as in 234 [26], illustrated in Figure 13b. The bis dithiocarbamate ligand $\mathbf{L}^{74}$ bridges two $\left(\mathrm{PEt}_{3}\right)_{2} \mathrm{Pt}$ entities in dicationic 235 [101], Figure 13c, and a similar structural motif is noted for 236 [101]. In the quaternary complex 237 [167], a $\mathrm{ClS}_{3}$ donor set is defined by the chelating dithiocarbamate ligand, a chloride and a S-bound DMSO molecule, Figure 13d. The complex molecules in 238 [168], 239 [166], 240 [169], 241 [169], 242 [169], and 243 [170], conform to the same structural motif as illustrated for 238 in Figure 13e.

The next series of structures are organoplatinum derivatives starting with the 244 [171] which is readily related to the previous series in that the chloride is now an iodide and the phosphane is replaced by the N-heterocyclic carbene, Figure 14a. The molecules in 245 [172], Figure 14b, 246 [172], and 247 [173] contain C,N-chelating orthometallated species as well as dithiocarbamate. In a variation, doubly orthometallated $2,2^{\prime}$-bipyridyl links two platinum centers in 248 [174], Figure 14c. Figure 14d represents 249 [175] and 250 [176] with $\mathrm{CPS}_{2}$ donor sets. In the final molecular structure to be described, the platinum atom of Figure $14 \mathrm{~b}$ is linked to $\mathrm{Pt}\left(\mathrm{C}_{6} \mathrm{~F}_{5}\right)_{2}$ via two diphenylphosphinato ligands resulting in a rare example of a $\mathrm{CNP}_{2} \mathrm{~S}_{2}$ donor set in 251 [177], Figure 14e. The last remaining structure to be described is a coordination polymer, i.e., in the crystal of 252 [172]. Here, the platinum atom is $\mathrm{C}, \mathrm{N}$-chelated by a benzo[h]quinolin-10-yl ligand with the square planar geometry completed by a chelating dithiocarbamate ligand. The latter is also connected to a silver cation which also forms connections to two platinum atoms to form a one-dimensional coordination polymer. 


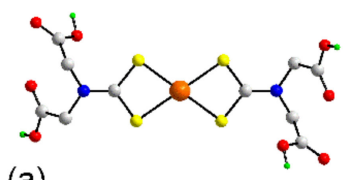

(a)

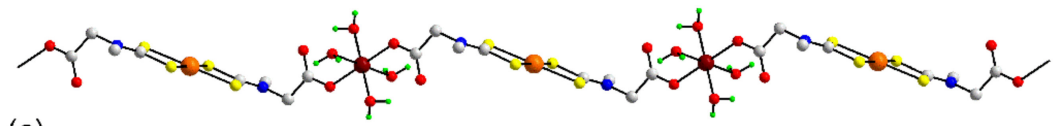

(c)

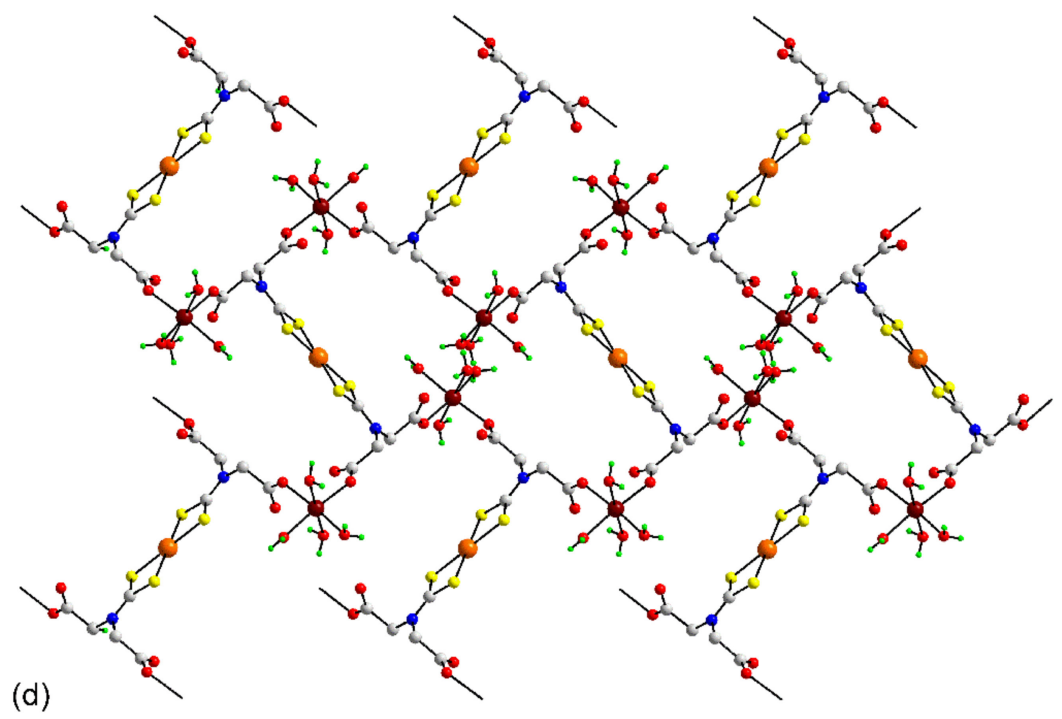

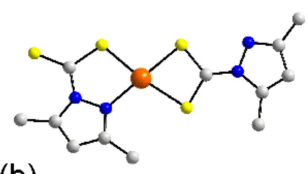

(b)

Figure 12. Molecular structures of platinum dithiocarbamate complexes: (a) $\mathrm{Pt}\left(\mathrm{L}^{\mathbf{5}}\right)_{2}$ (222) and (b) $\mathrm{Pt}\left(\mathbf{L}^{62}\right)_{2}(228)$, and coordination polymers in (c) $\left[\mathrm{Zn}\left(\mathrm{H}_{2} \mathrm{O}\right)_{4} \mathrm{Pt}\left(\mathbf{L}^{17}\right)_{2}\right]_{\mathrm{n}}$ in the crystal of 229 and (d) $\left\{\left[\mathrm{Zn}\left(\mathrm{H}_{2} \mathrm{O}\right)_{4}\right]_{2} \mathrm{Pt}\left(\mathbf{L}^{51}\right)_{2}\right\}_{\mathrm{n}}$ in 232 .

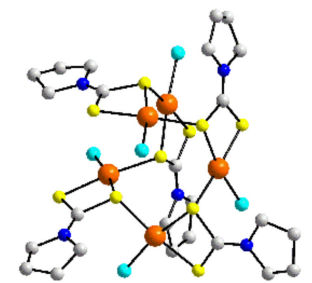

(a)

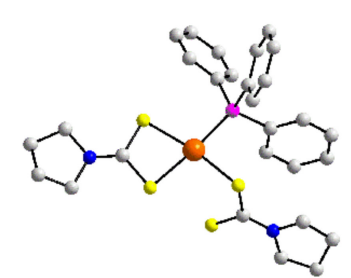

(b) 


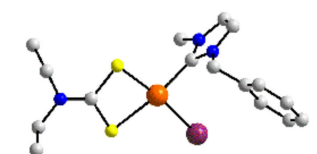

(a)

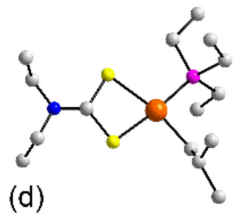

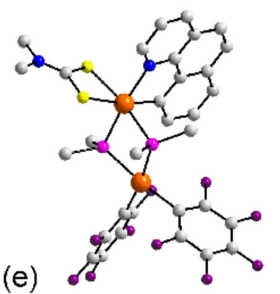

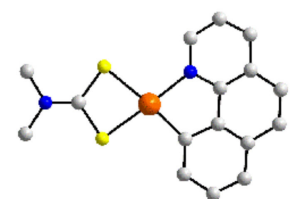

(b)

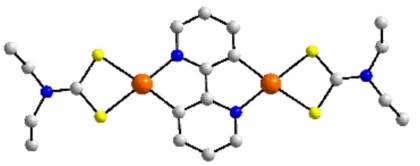

(c)

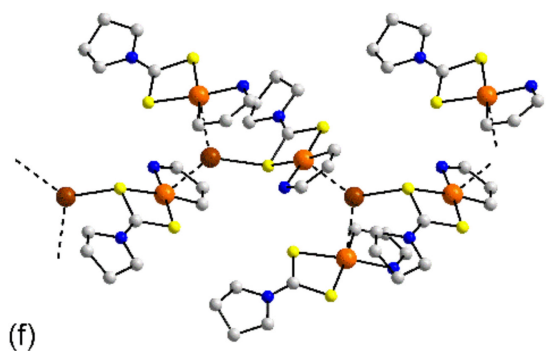

Figure 14. Molecular structures of platinum dithiocarbamate complexes: (a) $\mathrm{Pt}(\mathrm{bmi})\left(\mathbf{L}^{24}\right) \mathrm{I}(244)$, (b) $\left(\operatorname{Pt}(\mathrm{bzq})\left(\mathrm{L}^{8}\right)(\mathbf{2 4 5}),(\mathrm{c})\left[\mathrm{Pt}\left(\mathrm{L}^{24}\right)\right]_{2}\right.$ (bpy) in the crystal of 248 , (d) $\left(\mathrm{PEt}_{3}\right) \mathrm{Pt}\left(\mathrm{L}^{24}\right)\left(\mathrm{CH}_{2}(\mathrm{iPr})\right)(249)$, (e) $\mathrm{Pt}(\mathrm{bzq})\left(\mathrm{L}^{8}\right)\left(\mathrm{PPh}_{2}\right)_{2} \mathrm{Pt}\left(\mathrm{C}_{6} \mathrm{~F}_{5}\right)_{2}(\mathbf{2 5 1})$, and (f) the coordination polymer $\left\{\mathrm{Ag}\left[\mathrm{Pt}(\mathrm{bzq})\left(\mathrm{L}^{57}\right)\right]_{2}\right\}_{\mathrm{n}}$ in 252. In (f), only the atoms forming the chelate ring formed by the benzo[h]quinolin-10-yl ligand are shown.

\subsection{Nuclear Magnetic Resonance (NMR) Studies}

\subsection{1. ${ }^{1} \mathrm{H}$ NMR}

Solution ${ }^{1} \mathrm{H}$ NMR data has been reported for dithiocarbamate complexes for all platinum-group metals, with data for palladium(II) and ruthenium(II) complexes predominating. As a consequence of restricted rotation around the carbamoyl $\mathrm{C}-\mathrm{N}$ bond (see below), it is often the case that the dithiocarbamate ligand substituents give rise to two or, depending on the number of dithiocarbamate ligands and the local symmetry, occasionally more sets of resonances, which can complicate unambiguous assignment of signals, but conversely can also provide a wealth of useful information on the solution-state structures. The $\alpha$-hydrogen nuclei provide the most characteristic probe. The chemical shifts vary quite significantly and typically range between about 1.80 and $4.5 \mathrm{ppm}$, depending on both the dithiocarbamate itself and the metal moiety to which it is coordinated. Table 8 shows how the mean chemical shifts and the standard deviation of these shifts varies with metal ion; Figure 15 shows the mean and 95\% range (two standard deviations) for the shifts. Note that the data given in Table 8 and Figure 15 for ruthenium(II) are for dimeric complexes which are diamagnetic by virtue of spin pairing between the two ruthenium(II) centers. Although there is much overlap, Figure 15 reveals a clear trend in the mean shifts:

(i) Decrease down a Group,

(ii) Increase across a Period,

(iii) Increase with the oxidation state of the metal ion.

These trends are statistically significant (based on standard two-tailed $t$-tests).

Table 8. The mean ${ }^{1} \mathrm{H}$ chemical shifts reported for $\alpha$-hydrogen nuclei and the standard deviation of these shifts as a function of the metal ion.

\begin{tabular}{ccc}
\hline Metal Ion & Mean Shift, ppm & Sample Standard Deviation, ppm \\
\hline $\operatorname{Ru}(\mathrm{II})$ & 3.20 & 0.50 \\
$\mathrm{Ru}(\mathrm{III})$ & 3.63 & 0.37 \\
$\mathrm{Os}(\mathrm{II})$ & 2.47 & 0.63 \\
$\mathrm{Rh}(\mathrm{III})$ & 3.63 & 0.37 \\
$\mathrm{Ir}(\mathrm{III})$ & 3.29 & 0.44 \\
$\mathrm{Pd}(\mathrm{II})$ & 3.82 & 0.61 \\
$\mathrm{Pt}(\mathrm{II})$ & 3.83 & 0.50 \\
$\mathrm{Pt}(\mathrm{IV})$ & 3.51 & 0.26 \\
\hline
\end{tabular}




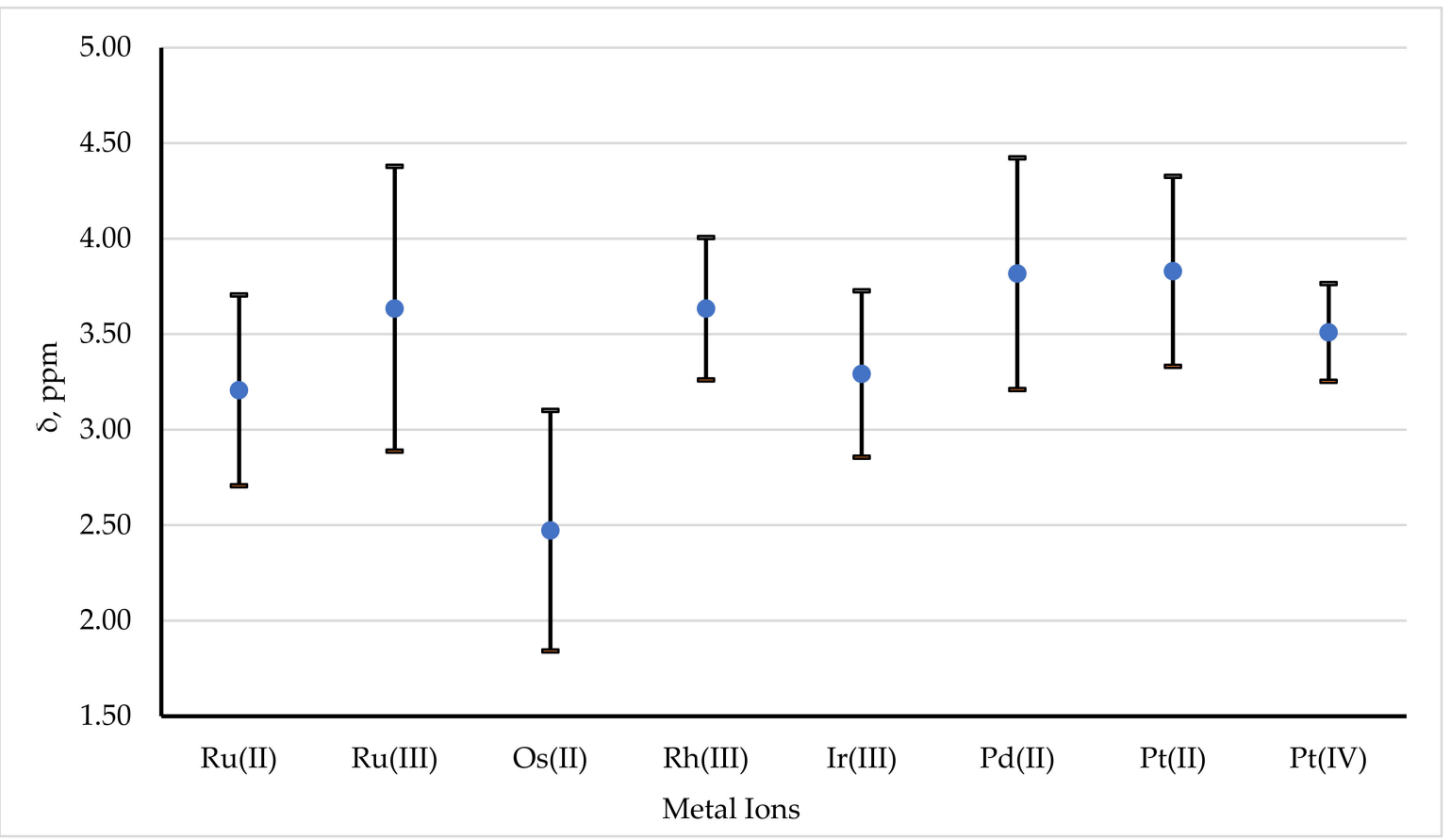

Figure 15. The mean and 95\% range (two standard deviations) for the ${ }^{1} \mathrm{H}$ chemical shifts reported for $\alpha$-hydrogen nuclei.

The ${ }^{1} \mathrm{H}$ NMR chemical shifts of the $\alpha$-hydrogens are also sensitive to the R-group of $\mathrm{R}_{2} \mathrm{NCS}_{2}{ }^{-}$, with those of benzyl groups and alicyclic groups, typically cyclohexyl, resonating at the higher end of the range and those of simply alkyl groups, such as $\mathrm{R}=\mathrm{Me}$ and Et at the lower end of the range. Thus, for example, in the complexes $\mathrm{Pd}\left(\mathrm{S}_{2} \mathrm{CNR}_{2}\right)\left(\eta^{3-} 2\right.$-methylallyl), $\mathrm{NR}_{2}=\mathrm{NMe}_{2}\left(\mathbf{L}^{8}\right), \mathrm{N}\left(\mathrm{CH}_{2} \mathrm{Ph}\right)_{2}\left(\mathbf{L}^{39}\right)$, and $\mathrm{N}(\mathrm{Me}) \mathrm{Cy}\left(\mathbf{L}^{15}\right)$, the signals due to $\alpha$-hydrogens of $\mathbf{L}^{39}$ and $\mathbf{L}^{15}$ are found at 4.92 and $3.7 \mathrm{ppm}$, respectively, while the $\mathrm{NCH}_{3}$ signals of $\mathbf{L}^{8}$ and $\mathbf{L}^{15}$ lie at 3.33 and $2.08 \mathrm{ppm}$, respectively [77]. Another comparative example may be found in reference [79]. The $\alpha$-hydrogen signals of piperazinedithiocarbamate ligands, ${ }_{-}^{-} \mathrm{S}_{2} \mathrm{CN}\left(\mathrm{CH}_{2} \mathrm{CH}_{2}\right)_{2} \mathrm{NR}$, are also generally found in the mid to upper end of the range; as, for example in [(p-tolyl $\left.)_{3} \mathrm{PPd}\left(\mathrm{L}^{69}\right) \mathrm{Cl}\right]: 3.60 \mathrm{ppm}$ [110]. Although less common, complexes of dithiocarbamates derived from primary amines have also been studied. Typical examples include $\mathrm{Pd}\left(\mathbf{L}^{2}\right)_{2}$ [73], $\mathrm{Pd}\left(\mathbf{L}^{4}\right)_{2}$ [73] and $\left\{\mathrm{Me}_{3} \mathrm{Pt}\left(\mathbf{L}^{2}\right)\right\}_{2}$ [178]. The $\mathrm{NH}$ hydrogen signal is found in the range 6.82 to $10.95 \mathrm{ppm}$. Limited data frustrate attempts to identify any trends in the shifts.

Routinely, NMR spectroscopy is generally restricted to diamagnetic compounds because the presence of unpaired electrons can substantially reduce relaxation times, which can lead to very broad and often featureless spectra. However, paramagnetism also extends the normal chemical shift range, and in so doing can provide useful additional structural information. The rate of relaxation and the chemical shift of a nuclide in the presence of a paramagnetic center are related to the distance from that paramagnetic center. At short separations, the high rates of relaxation broaden the signals to such an extent that they are not detectable, whilst at longer ranges, the effects may be too small to give appreciable effects. Between these limits-the Goldilocks zone-observable, but widely dispersed signals are seen. These signals can be used to infer more detailed information about the structure. Dithiocarbamate complexes of ruthenium(II) are a good example, with the $\alpha$-hydrogens of the dithiocarbamate, often lying in the Goldilocks zone. ${ }^{1} \mathrm{H}$ NMR data for a series of ruthenium(III) dithiocarbamate complexes are given in Table 9. 
Table 9. ${ }^{1} \mathrm{H}$ Chemical shifts of $\alpha$-hydrogen nuclei for a series of ruthenium(III) dithiocarbamate complexes.

\begin{tabular}{ccc}
\hline Complex & $\boldsymbol{\delta}, \mathbf{p p m}$ & Ref. \\
\hline$\left[\mathrm{Ru}\left(\mathbf{L}^{8}\right)_{3}\right]$ & 28.85 & {$[25]$} \\
& 28.70 & {$[32]$} \\
{$\left[\mathrm{Ru}\left(\mathbf{L}^{57}\right)_{3}\right]$} & $45.03,36.28$ & {$[25]$} \\
{$\left[\mathrm{Ru}\left(\mathbf{L}^{\mathbf{1 9}}\right)_{3}\right]$} & $44.40,36.00$ & {$[32]$} \\
{$\left[\mathrm{Ru}\left(\mathbf{L}^{\mathbf{2 0}}\right)_{3}\right]$} & $26.80,26.70,21.83,14.63$ & {$[25]$} \\
{$\left[\mathrm{Ru}\left(\mathbf{L}^{21}\right)_{3}\right]$} & $26.70,26.23,21.92,14.55$ & {$[25]$} \\
$\alpha-\left[\mathrm{Ru}_{2}\left(\mathbf{L}^{8}\right)_{5}\right] \mathrm{Cl}$ & $26.82,26.43,21.32,14.40$ & {$[25]$} \\
$\beta-\left[\mathrm{Ru}_{2}\left(\mathbf{L}^{8}\right)_{5}\right] \mathrm{Cl}$ & $3.55,3.52,3.50,3.20,2.99$ & {$[25]$} \\
$\alpha-\left[\mathrm{Ru}_{2}\left(\mathbf{L}^{57}\right)_{5}\right] \mathrm{Cl}$ & $3.62,3.46,3.47,3.17,2.93$ & {$[32]$} \\
$\beta-\left[\mathrm{Ru}_{2}\left(\mathbf{L}^{57}\right)_{5}\right] \mathrm{Cl}$ & $3.55,3.54,3.51,3.20,3.19$ & {$[25]$} \\
$\alpha-\left[\mathrm{Ru}_{2}\left(\mathbf{L}^{19}\right)_{5}\right] \mathrm{Cl}$ & $4.05-3.37$ & {$[32]$} \\
$\beta-\left[\mathrm{Ru}_{2}\left(\mathbf{L}^{\mathbf{2 0}}\right)_{5}\right] \mathrm{Cl}$ & $4.25-3.15$ & {$[32]$} \\
$\alpha-\left[\mathrm{Ru}_{2}\left(\mathbf{L}^{21}\right)_{5}\right] \mathrm{Cl}$ & $3.98-3.40$ & {$[25]$} \\
& $4.60-4.00,3.48-3.14$ & {$[25]$} \\
\hline
\end{tabular}

In the case of $\mathrm{Ru}\left(\mathrm{L}^{8}\right)_{3}$, the magnetic moment was calculated from the ${ }^{1} \mathrm{H}$ NMR shifts, using the Evans' Method [179]. The effective magnetic moment, $\mu_{\text {eff }}$, was found to be $1.92 \mu_{\mathrm{B}}$, at room temperature, consistent with a low-spin $\mathrm{d}^{5}$ electronic configuration: i.e., with a single unpaired electron [25]. For complexes of general formula M(S-S $)_{3}$ for $\mathrm{S}-\mathrm{S}=$ dithiocarbamate, there exists the possibility of two isomeric forms, namely L-M(L-L) 3 and D-M(S-S $)_{3}$, Figure 16.<smiles>C1CS[As]2(SC1)SCCSC21SCCS1</smiles><smiles>C1CSC2(S1)SCCS2</smiles>

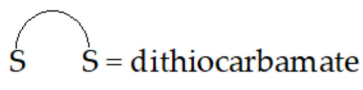

Figure 16. L- and D-forms of $\left[\mathrm{M}(\mathrm{L}-\mathrm{L})_{3}\right]$.

The two isomers are exchanged by inversion at the metal center. In the case of the $\mathrm{Ru}(\mathrm{III})$ complexes, both the barrier to inversion and the barrier to rotation about the dithiocarbamate C-N bond (see below) are such that exchange is slow on the NMR time scale at room temperature. Consequently, the NMR spectra in complexes of unsymmetrical dithiocarbamate ligands can be quite complex, with different (C-N) rotational isomers observed for each of the $\mathrm{L}$ - and the D-configurations. In the case of $\mathrm{Ru}\left(\mathrm{L}^{\mathbf{6 1}}\right)_{3}$, for example [180], 12 sets of signals due to the $\alpha$-hydrogen nuclei are observed in the ${ }^{1} \mathrm{H}$ NMR spectrum in the region $50-20 \mathrm{ppm}$.

In the majority of cases, the dithiocarbamate ligand is coordinated to the metal center via both sulfur atoms in an essentially symmetric fashion. However, there are examples of where the dithiocarbamate is coordinated via one sulfur atom only. For example, in $\left[\mathrm{Tp} \mathrm{p}^{*} \mathrm{Rh}\left(\eta^{2}-\mathrm{L}^{24}\right)\left(\eta^{1}-\mathrm{L}^{24}\right)\right]\left(\mathrm{Tp} \mathrm{p}^{*}=\mathrm{HB}(3,5-\mathrm{pyrazol}-1-\mathrm{yl})\right)$ [68], and [Cp* $\left.\mathrm{Rh}\left(\eta^{2}-\mathrm{L}^{24}\right)\left(\eta^{1}-\mathrm{L}^{24}\right)\right]$ $\left(\mathrm{Cp}^{*}=\right.$ pentamethylcyclopentadienyl) [181], one of the two diethyldithiocarbamate ligands is symmetrically bound via both $S$ atoms, while the other acts as a monodentate ligand. The different coordination modes are readily distinguished because, in the case of the bidentate bound ligand, the lack of symmetry along the $\mathrm{S}_{2} \mathrm{C}-\mathrm{N}$ bond results in two sets of signals being observed for the ethyl group in the symmetrically bound ligand, but one set for the monodentate bound ligand: the methylene $\left(\mathrm{NCH}_{2}\right)$ hydrogens appear as a doublet of quartets in the $\kappa^{2}$-bound ligand and as a simple quartet in the $\kappa^{1}$-bound ligand. In the case of $\left[C \mathrm{p}^{*} \mathrm{Rh}\left(\mathrm{K}^{2}-\mathrm{L}^{24}\right)\left(\mathrm{\kappa}^{1}-\mathrm{L}^{24}\right)\right]$, coalescence of the signals is observed above $c a 350 \mathrm{~K}$ indicating 
exchange between the $\kappa^{2}$ - and $\kappa^{1}$-bound ligands [181]: such exchange was not observed in $\left[T p^{*} \operatorname{Rh}\left(\kappa^{2}-L^{24}\right)\left(\kappa^{1}-L^{24}\right)\right]$. One final point to note is that the ${ }^{1} \mathrm{H}$ signals of the dithiocarbamate ligands such as piperazinedithiocarbamate or mono- or dicyclohexyl-dithiocarbmate may also be affected by dynamic phenomena of the ring. Thus, for example, it is not untypical for the piperazine $\left(\mathrm{S}_{2} \mathrm{C}\right) \mathrm{NCH}_{2}$ - signals to be broadened by ring flexing [55].

Although agostic interactions between the $\alpha$-hydrogens of dithiocarbamate ligands, $\mathrm{S}_{2} \mathrm{CN}(\mathrm{R}) \mathrm{CH}\left(\mathrm{R}^{\prime}\right)$, and the metal center may be observed in the solid-state, such interactions are not generally evident in solution, at least under ambient conditions. Attempts to establish the presence of agostic interactions at lower temperatures have met with mixed success. Thus, for example, ${ }^{1} \mathrm{H}$ NMR of a solution $\mathrm{Ni}\left(\mathrm{L}^{40}\right)_{2}$, cooled to liquid nitrogen temperatures failed to reveal any evidence of the agostic interactions observed in the crystal structure of the complex [86]. Whereas, for the related palladium(II) complex, $\mathrm{Pd}\left(\mathrm{L}^{41}\right)_{2}$ for [86], the singlet due to the $\alpha$-hydrogen nuclei of the $\mathrm{CH}_{2} \mathrm{C}_{5} \mathrm{H}_{4} \mathrm{~N}-3$, split into two equally intense signals at $233 \mathrm{~K}$, consistent with one of the methylene hydrogen atoms being involved in an agostic interaction with the metal center. The ${ }^{1} \mathrm{H}$ NMR sub-spectra due to the dithiocarbamate ligand in these complexes can, overall, thus provide a wealth of structural information, and hence be of intrinsic value beyond just routine characterization.

\section{Barrier to C-N Bond Rotation}

The significant contribution of resonance form (II) (Figure 1) lends substantial double bond character to the $\mathrm{S}_{2} \mathrm{C}-\mathrm{N}$ bond, restricting rotation to an extent that, where symmetry allows, different rotomeric species are often visible in the NMR spectra at ambient temperature. Thus, for example, in cis- $\left[\mathrm{Os}(\mathrm{CO})_{2}\left(\mathbf{L}^{8}\right)_{2}\right]$, although the two dithiocarbamate ligands are equivalent, there is no plane of symmetry aligned along the $\mathrm{C}-\mathrm{N}$ bond vector, rendering the methyl groups in each ligand inequivalent. Two equally intense signals, each of which integrates to six hydrogens, are observed for the $\mathrm{NCH}_{3}$ groups at ambient temperature. These signals broaden on warming and eventually coalesce at $c a 90{ }^{\circ} \mathrm{C}$ [130]. Similarly, the methylene protons of $\left\{\operatorname{Pd}\left(\mathbf{L}^{13}\right)_{2}\right\}$ [78] are also inequivalent and, due to the restricted rotation about the $\mathrm{C}-\mathrm{N}$ bonds are distinguishable in the NMR spectrum. Two doublets, each of integral 2, are observed at 4.75 and $5.21 \mathrm{ppm}\left({ }^{2} J_{\mathrm{HH}}=7 \mathrm{~Hz}\right)$. Well known as the barrier to rotation is, there remain relatively few studies on the magnitude of that barrier: contemporary data for platinum-group metal complexes are collected in Table 10.

Table 10. Contemporary data for the barrier to rotation about the $\mathrm{C}-\mathrm{N}$ bonds of platinum-group metal complexes.

\begin{tabular}{|c|c|c|c|}
\hline Complex & $\Delta \mathrm{G}, \mathrm{kJ} \mathrm{\textrm {mol } ^ { - 1 }}$ & $v(C-N), \mathrm{cm}^{-1}$ & Ref. \\
\hline$\left[\mathrm{Ru}(\mathrm{CO})_{2}\left(\mathbf{L}^{\mathbf{8}}\right)_{2}\right]$ & $78 \pm 1$ & Not reported & [182] \\
\hline$\left[\mathrm{Ru}(\mathrm{CO})\left(\mathrm{PEt}_{3}\right)\left(\mathbf{L}^{\mathbf{8}}\right)_{2}\right]$ & $74 \pm 1$ and $71 \pm 1$ & Not reported & [46] \\
\hline$\left[\mathrm{Os}(\mathrm{CO})_{2}\left(\mathbf{L}^{8}\right)_{2}\right]$ & $74.2 \pm 0.5$ & Not reported & [130] \\
\hline$\left[\left\{\mathrm{PtMe}_{3}\left(\mathbf{L}^{\mathbf{1}}\right)\right\}_{2}\right]$ & $71.43 \pm 0.14$ & 1609 & [178] \\
\hline$\left[\left\{\mathrm{PtMe}_{3}\left(\mathrm{~L}^{\mathbf{8}}\right)\right\}_{2}\right]$ & $81.54 \pm 0.19$ & 1530 & [178] \\
\hline$\left[\left\{\mathrm{PtMe}_{3}\left(\mathrm{~L}^{24}\right)\right\}_{2}\right]$ & $82.89 \pm 0.56$ & 1516 & [178] \\
\hline$\left[\left\{\mathrm{PtMe}_{3}\left(\mathbf{L}^{2}\right)\right\}_{2}\right]$ & $72.27 \pm 0.05$ & 1517 & [178] \\
\hline$\left[\left\{\mathrm{PtMe}_{3}\left(\mathrm{~L}^{3}\right\}_{2}\right]\right.$ & $66.36 \pm 0.05$ & 1518 & [178] \\
\hline \multirow[t]{2}{*}[\{\mathrm{PtMe}_{3}(\mathrm{L}^{4})\}_{2}]{} & $65.61 \pm 0.01$ & 1527 & [178] \\
\hline & $86.54 \pm 0.29$ & & \\
\hline \multirow{2}{*}[\{\mathrm{PtMe}_{3}(\mathbf{L}^{\mathbf{14}})\}_{2}]{} & $87.24 \pm 0.27$ & 1489 & [178] \\
\hline & $87.15 \pm 0.35$ & & \\
\hline
\end{tabular}

Table 10 also includes a comparison of the $\mathrm{C}-\mathrm{N}$ stretching frequencies, where available, with the barrier to rotation. This relationship is depicted graphically in Figure 17. Figure 17 shows that the $\mathrm{C}-\mathrm{N}$ stretching frequencies are very loosely, but never-the-less negatively correlated with higher barriers to $\mathrm{C}-\mathrm{N}$ bond rotation: higher barriers are more associated with lower $\mathrm{C}-\mathrm{N}$ stretching frequencies. This is contrary to expectation given that higher barriers should be associated with an increased double bond character, and thence would 
be expected to correlate with higher $\mathrm{C}-\mathrm{N}$ stretching frequencies. However, with the very limited data available more inquiry into this relationship would be of value.

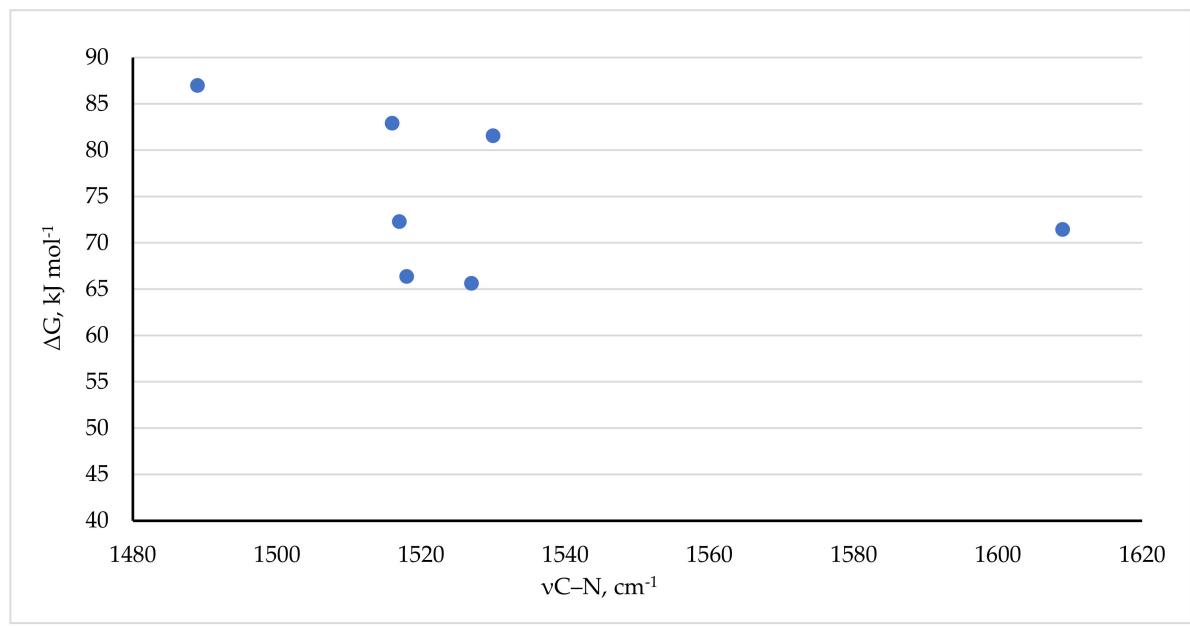

Figure 17. Correlation of $v_{C-N}$ with the barrier to $\mathrm{C}-\mathrm{N}$ bond rotation.

\subsection{2. ${ }^{13} \mathrm{C} \mathrm{NMR}$}

${ }^{13} \mathrm{C}$ NMR data are, unsurprisingly, more sparse than ${ }^{1} \mathrm{H}$ NMR data. The quaternary carbamoyl carbon atom, $\mathrm{S}_{2} \mathrm{CNR}_{2}$, is most characteristic, although it is not always observed because of its long relaxation times. For the compounds discussed in this review, shifts typically range between $c a 200$ and 215 ppm (mean ca 208 ppm) and, compared to the corresponding signal in the free ligand, is typically shifted to a lower frequency by around $5 \mathrm{ppm}$, due to a reduction in the delocalization in the $\mathrm{S}_{2} \mathrm{C}-\mathrm{N}$ system (i.e., increases the contribution of structures I and III, Figure 1). As would logically be expected, since coordination reduces delocalization, the $v_{\mathrm{C}-\mathrm{N}}$ bond stretching in the infrared, which is normally in the range $1320-1600 \mathrm{~cm}^{-1}$, generally increases on coordination [183]. Whilst the carbamoyl moiety provides the most-ready probe for confirming metal complexation, the wider dispersion of ${ }^{13} \mathrm{C}$ chemical shifts, as compared to those of ${ }^{1} \mathrm{H}$, mean that the ${ }^{13} \mathrm{C}$ signals of the nitrogen substituents, $\mathrm{S}_{2} \mathrm{CNR} R_{2}$, are also of utility. The ${ }^{13} \mathrm{C}$ NMR shifts of the $\mathrm{N}$-alkyl substituent groups are to be found in the region $12-55 \mathrm{ppm}$, with those attached directly to the $\mathrm{N}$-atom $(\alpha-C)$ found at the high-frequency end of this range: $45-55 \mathrm{ppm}$; while $\beta$ - and $\gamma$-carbon nuclides are typically in the ranges $20-30$ and $12-20 \mathrm{ppm}$, respectively. These distinct chemical shift ranges make the assignment of the ${ }^{13} \mathrm{C}$ NMR spectra of alkyl-substituted dithiocarbonate complexes a relatively straightforward and unambiguous process. The ${ }^{13} \mathrm{C}$ NMR shifts of $\mathrm{N}$-aryl substituent groups are, as would be expected, less well separated and typically observed in the region $120-150 \mathrm{ppm}$ : $\mathrm{C}_{\text {ipso }} 130-160 \mathrm{ppm} ; \mathrm{C}_{\text {ortho }} 122-132 \mathrm{ppm}$; $\mathrm{C}_{\text {meta }}$ 125-130 ppm; $\mathrm{C}_{\text {para }}$ 120-135 ppm.

Solid-state NMR (SSNMR) studies, usually ${ }^{13} \mathrm{C} C \mathrm{CP}-\mathrm{MAS}$, have been reported for a number of complexes $[98,164,175]$. Unsurprisingly, the SSNMR spectra are generally more complex than the corresponding solution NMR spectra because the three-dimensional crystalline environment effectively reduces the symmetry of the molecules. Thus, for example, the ${ }^{13} \mathrm{C} C \mathrm{CP}-\mathrm{MAS}$ SSNMR spectrum of $\mathrm{Pt}\left(\mathbf{L}^{80}\right)_{2}$ reveals the dithiocarbamate ligands to be inequivalent in the solid-state; the carbamoyl carbon atoms $\left(\mathrm{S}_{2} \mathrm{CN}<\right)$ give rise to a 1:5:5:1 pseudo-quartet at ca $209.6 \mathrm{ppm}$, resulting from overlapping 1:4:1 triplets (the triplet fine structure arises from coupling to ${ }^{195} \mathrm{Pt}$ : $\mathrm{I}=1 / 2 ; 33.8 \%$ abundance), whereas in solution a single signal is observed [164]. The same patterns are observed in the solid-state ${ }^{13} \mathrm{C}$ spectra of $\mathrm{Pt}\left(\mathbf{L}^{29}\right)_{2}$ and $\mathrm{Pt}\left(\mathbf{L}^{34}\right)_{2}$ [155]. Although the symmetry is lower in the solid-state, it is clear that the basic molecular structure is the same in the solid-state and solution in these complexes. Likewise, the structure of silica-supported $\left[\mathrm{Pd}\left(\mathrm{Ph}_{2} \mathrm{P}\left(\mathrm{CH}_{2}\right)_{3} \mathrm{PPh}_{2}\right)\left(\mathrm{L}^{24}\right)\right] \mathrm{BF}_{4}$ has been shown by SSNMR to be largely unaffected, compared to the solution structure [98]. 


\subsection{3. ${ }^{195} \mathrm{Pt} \mathrm{NMR}$}

${ }^{195} \mathrm{Pt}$ NMR data are available for some platinum(II) and platinum(IV) dithiocarbamate complexes. Data are collated in Table 11.

Table 11. ${ }^{195} \mathrm{Pt}$ NMR data reported for selected $\mathrm{Pt}(\mathrm{II})$ and $\mathrm{Pt}(\mathrm{IV})$ dithiocarbamate complexes.

\begin{tabular}{|c|c|c|c|}
\hline Complex & $\delta, \mathrm{ppm}^{\mathrm{a}}$ & $\delta, \mathrm{ppm}^{\mathrm{b}}$ & Ref. \\
\hline$\left[\mathrm{Pt}(\mathrm{pbt})\left(\mathrm{L}^{24}\right)\right]$ & 686 & - & [143] \\
\hline$\left[\mathrm{Pt}\left(\mathbf{L}^{18}\right)_{2}\right]$ & 626 & - & [161] \\
\hline$\left[\mathrm{Pt}\left(\mathrm{L}^{59}\right)_{2}\right]$ & 720 & - & [161] \\
\hline $\mathrm{Zn}\left[\mathrm{Pt}\left(\mathbf{L}^{58}\right)_{2}\right]$ & 793 & - & [161] \\
\hline$\left[\mathrm{Pt}\left(\mathbf{L}^{52}\right)_{2}\right]$ & 603 & - & [161] \\
\hline $\mathrm{Zn}\left[\mathrm{Pt}\left(\mathbf{L}^{53}\right)_{2}\right]$ & 614 & - & [161] \\
\hline $\mathrm{Zn}_{2}\left[\mathrm{Pt}\left(\mathbf{L}^{51}\right)_{2}\right]$ & 678 & - & [161] \\
\hline $\begin{array}{l}{[\mathrm{WPt}(\mu-} \\
\left.\mathrm{C})\left(\mathrm{L}^{24}\right)(\mathrm{CO})_{2}\left(\mathrm{PPh}_{3}\right)\left(\mathrm{Tp}^{*}\right)\right]\end{array}$ & 694 & - & [176] \\
\hline$\left[\mathrm{Pt}(\mathrm{pmea})\left(\mathrm{L}^{24}\right)\right]$ & 810 & - & [173] \\
\hline$\left[\mathrm{PtMeI}(\right.$ pmea $\left.)\left(\mathbf{L}^{24}\right)\right]$ & 1333 & - & [173] \\
\hline$\left[\left\{\mathrm{PtMe}_{3}\left(\mathbf{L}^{\mathbf{1}}\right)\right\}_{2}\right]$ & 1416 & - & [178] \\
\hline$\left[\left\{\mathrm{PtMe}_{3}\left(\mathrm{~L}^{8}\right)\right\}_{2}\right]$ & 1577 & - & [178] \\
\hline$\left[\left\{\mathrm{PtMe}_{3}\left(\mathbf{L}^{24}\right)\right\}_{2}\right]$ & 1564 & - & [178] \\
\hline$\left[\left\{\mathrm{PtMe}_{3}\left(\mathbf{L}^{2}\right)\right\}_{2}\right]$ & $1623,1609,1598,1587$ & - & [178] \\
\hline$\left[\left\{\mathrm{PtMe}_{3}\left(\mathrm{~L}^{3}\right\}_{2}\right]\right.$ & $1626,1609,1607,1596$ & - & [178] \\
\hline$\left[\left\{\mathrm{PtMe}_{3}\left(\mathrm{~L}^{4}\right\}_{2}\right]\right.$ & $1632,1600,1587,1582$ & - & [178] \\
\hline$\left[\left\{\mathrm{PtMe}_{3}\left(\mathrm{~L}^{\mathbf{1 4}}\right)\right\}_{2}\right]$ & $1584,1575,1569,1559$ & 1601 & [178] \\
\hline
\end{tabular}

a Spectra recorded at ambient temperature in solution. Chemical shifts quoted relative to the absolute frequency scale $\Xi\left({ }^{195} \mathrm{Pt}\right)=21.4 \mathrm{MHz}$, and, where appropriate, have been converted from originally published data: $\delta\left[\mathrm{PtCl}_{6}\right]^{2-}+4522=\Xi\left({ }^{195} \mathrm{Pt}\right)$. Conversion factor is taken from [184]. ${ }^{\mathrm{b}}$ Solid-state NMR, recorded at ambient temperature. Chemical shifts quoted relative to the absolute frequency scale $\Xi\left({ }^{195} \mathrm{Pt}\right)=21.4 \mathrm{MHz}$.

For the platinum(II) complexes, the chemical shifts range from 603 to $810 \mathrm{ppm}$, as compared to 1416 to $1632 \mathrm{ppm}$ for the platinum(IV) complexes. The higher chemical shifts of the platinum(IV) complexes are consistent with the general observation that the resonance frequency increases with the oxidation state [184]. Similarly, the trend to a higher frequency in the platinum(II) complexes on ionization (removal of $\mathrm{H}^{+}$) is consistent with the higher charge at the metal center.

\subsection{Applications}

\subsubsection{Biological Applications}

Perhaps unsurprisingly, one of the most common areas of the potential application of dithiocarbamate complexes to be explored is that related to their anti-cancer properties. Ever since cisplatin, cis-[ $\left[\mathrm{PtCl}_{2}\left(\mathrm{NH}_{3}\right)_{2}\right]$, was first approved for use in the treatment of testicular and ovarian cancers by the US food and Drug Administration in 1978 [185], the chemotherapeutic applications of platinum and, more generally, platinum group metal compounds have been the subject of ongoing investigation. Given that organic derivatives of dithiocarbamates, RSC(S)NR' ${ }_{2}$, also display antitumor activity [186], the exploration of anti-cancer properties of platinum-group metal dithiocarbamate complexes is an obvious avenue for research [187].

The half-maximal inhibitory concentration, $\mathrm{IC}_{50}$, is a commonly used measure of the effectiveness of a compound at inhibiting a particular biological function, such as cell proliferation. The in vitro cytotoxicities of those platinum-group metal complexes described in this review against various human cancer cell lines are given in Table 12. Given that the effectiveness of complexes has been investigated against a variety of different cell lines and with many experiments conducted under different laboratory conditions, it is hard to draw general conclusions from the data. Where a common cell line has been used (HeLa; cervical cancer cell line), data indicate that the ruthenium complexes are significantly more active (lower $\mathrm{IC}_{50}$ ) than either palladium or platinum. The sparse data 
and diversity of cell lines used in reported cases suggest that some more systematic studies on the antitumor activity of platinum group metal dithiocarbamate complexes would add significantly to the body of literature.

DNA binding studies have also been carried out to explore the mechanism of the antitumor activity. The DNA binding of complexes of general formula $\left(\mathrm{PR}_{3}\right) \mathrm{Pd}\left(\mathrm{S}_{2} \mathrm{CNR}_{2}\right) \mathrm{Cl}$, $\mathrm{PR}_{3}=\mathrm{PPh}_{3}, \mathrm{PPh}_{2} \mathrm{Cl}, \mathrm{PPh}_{2}$ (tert-Bu), $\mathrm{PPh}_{2}\left(\mathrm{CH}_{2} \mathrm{Ph}\right), \mathrm{PPh}_{2}$ (o-tolyl), $\mathrm{PPh}_{2}$ (p-tolyl), $\mathrm{PPh}_{2}\left(\mathrm{C}_{6} \mathrm{H}_{4}\right.$ $\mathrm{OMe}-\mathrm{O}) ; \mathrm{R}=\mathrm{Me}\left(\mathbf{L}^{8}\right)$, Et $\left(\mathbf{L}^{24}\right)$, n-Pr $\left(\mathbf{L}^{28}\right)$, n-Bu $\left(\mathbf{L}^{31}\right)$, 2-methoxyethyl $\left(\mathbf{L}^{26}\right)$, have been probed by cyclic voltammetry $[103,115]$. The cyclic voltammograms of the complexes display a single cathodic peak due to an irreversible one-electron reduction of the complexes. The addition of DNA more normally causes a diminution in the cathodic peak height and may also induce a shift in the position of the peak, consistent with DNA binding. In some complexes, DNA binding has been shown to lead to increased cathodic peak height, indicating DNA may also catalyze the electron transfer process [103]. Binding constants [103] were found to be in the range $6.16 \times 10^{2}-7.05 \times 10^{6} \mathrm{M}^{-1}$, with the corresponding free energies of activation being in the range -16 to $-39 \mathrm{~kJ} \mathrm{~mol}^{-1}$. The greatest binding affinity was found for $\left[\mathrm{PPh}_{2}(p\right.$-tolyl $\left.\left.) \mathrm{Pd}\left(\mathrm{L}^{31}\right)_{2}\right\}(\mathrm{Cl})\right]$ and was attributed to the presence of the hydrophobic butyl groups on the dithiocarbamate.

UV-Vis spectroscopy also provides a readily accessible probe for DNA binding studies. The UV-vis spectra typically display bands in the region $220-460 \mathrm{~nm}$, attributable to both charge transfer (CT: metal-to-ligand and ligand-to-metal), intraligand $\pi \rightarrow \pi^{*}$ and metal centered $d$ - $d$ transitions. The CT bands are distinguishable because of their high molar extinction coefficients $[25,180]$. The addition of increasing concentrations of DNA leads to a decrease in absorption peak intensity, from which the free energies of interaction can be calculated, with complex-specific negative values indicating spontaneous interaction between the DNA and the complexes [109,188,189]. In general, the DNA-complex appears to be stable over a prolonged period (at least $48 \mathrm{~h}$ ). For further information, the interested reader is referred to [190-194] being reviews of platinum group dithiocarbamates and anti-cancer potential.

Although less well studied than their anti-cancer properties, platinum group metal dithiocarbamate complexes are also known to exhibit antifungal properties. The antifungal properties of the palladium(II) and platinum(II) complexes $\mathrm{M}\left[\mathrm{S}_{2} \mathrm{CN}(\mathrm{Me}) \mathrm{R}\right]_{2}$, where $\mathrm{R}=$ 2,3-dimethoxyethyl $\left(\mathbf{L}^{\mathbf{1 6}}\right)$ or 1,3-doxolane-2-methyl $\left(\mathbf{L}^{\mathbf{2 3}}\right)$, have been reported [80]. All four complexes were found to be active against $A$. flavus, $A$. niger, and $A$. parasiticus: the complexes are more active than the drug Nyastin, but less active than miconazole nitrate, which is known to display extensive biocide action. In general, the palladium complexes displayed similar or greater activity. In the case of the palladium complexes, those of $\mathbf{L}^{\mathbf{2 3}}$ were generally more active, whereas in the platinum complexes, those of $\mathbf{L}^{\mathbf{1 6}}$ were more active [80]. These preliminary studies suggest that the antifungal behavior of platinum-group metal dithiocarbamate complexes would warrant further study.

Another potential application offered by dithiocarbamates being their use as an antibacterial agent, as shown by the inhibitory effect of pyrrolidine dithiocarbamate against bacterial strains $P$. gingivalis, A. actinomycetemcomitans, $S$. aureus, and E. coli and the enhanced activities resulted from the coadministration of $\mathrm{ZnCl}_{2}$ [195,196]. Significantly more investigations have been performed on the antibacterial potential of heavy element dithiocarbamate compounds with the area being reviewed very recently [197]. Among which, a series of six heteroleptic palladium(II) dithiocarbamates of general formula $\left(\mathrm{PR}_{3}\right) \mathrm{Pd}\left(\mathrm{S}_{2} \mathrm{CNR}_{2}\right) \mathrm{Cl}, \mathrm{PR}_{3}=\mathrm{PCy}, \mathrm{PPh}_{2}\left(\mathrm{CH}_{2} \mathrm{Ph}\right), \mathrm{PPh}_{2}\left(\mathrm{C}_{5} \mathrm{H}_{4} \mathrm{~N}-2\right), \mathrm{PPh}_{2}(\mathrm{~m}$-tolyl$)$, $\mathrm{PPh}_{2}$ (p-tolyl), $\mathrm{PPh}_{2}\left(\mathrm{C}_{6} \mathrm{H}_{4} \mathrm{OMe}-\mathrm{o}\right) ; \mathrm{R}=\mathrm{Me}\left(\mathbf{L}^{8}\right), \mathrm{Et}\left(\mathbf{L}^{24}\right), 2$-methoxyethyl $\left(\mathbf{L}^{26}\right)$ and $\mathrm{Cy}\left(\mathbf{L}^{47}\right)$ demonstrated antibacterial potency against two Gram-negative (E. coli and K. pneumoniae) and three Gram-positive bacterial strains (S. epidermidis, S. aureus, and B. subtilis) [117]. The above study further suggested dithiocarbamates of increased lipophilicity presented improved efficacy owing to the better permeability through the cell membrane of the bacterium. 
Table 12. In vitro cytotoxicity of platinum-group metal dithiocarbamate complexes.

\begin{tabular}{|c|c|c|c|c|c|c|c|c|c|c|c|c|c|c|c|}
\hline \multirow{2}{*}{ Complex $^{a}$} & \multicolumn{14}{|c|}{$\mathrm{IC}_{50} / \mu \mathrm{M}^{\mathrm{b}}$} & \multirow{2}{*}{ Ref. } \\
\hline & LU & MCF7 & Heap-1c1c7 & PC-3 & MDA-MB-231 & HepG2/CTR & HepG2/SB3 & HeLa & HL60 & Daudi & LoVo & A549 & HTB-81 & MRC-5 & \\
\hline$\beta-\left[\mathrm{Ru}_{2}\left(\mathrm{~L}^{57}\right)_{5}\right] \mathrm{Cl}$ & - & - & - & - & - & $0.62(0.08)$ & $0.59(0.01)$ & $0.45(0.02)$ & - & - & - & - & - & - & [180] \\
\hline$\beta-\left[\mathrm{Ru}_{2}\left(\mathrm{~L}^{63}\right)_{5}\right] \mathrm{Cl}$ & - & - & - & - & - & $0.66(0.01)$ & $0.75(0.02)$ & $0.46(0.04)$ & - & - & - & - & - & - & [180] \\
\hline$\beta-\left[\mathrm{Ru}_{2}\left(\mathrm{~L}^{60}\right)_{5}\right] \mathrm{Cl}$ & - & - & - & - & - & $9.2(2)$ & $>10$ & $3.95(0.07)$ & - & - & - & - & - & - & [180] \\
\hline$\beta-\left[\mathrm{Ru}_{2}\left(\mathbf{L}^{61}\right)_{5}\right] \mathrm{Cl}$ & - & - & - & - & - & $1.28(0.05)$ & $0.90(0.02)$ & $0.27(0.01)$ & - & - & - & - & - & - & [180] \\
\hline$\left[\mathrm{PPh}_{3} \mathrm{Ru}\left(\eta^{1}-\mathrm{ClL}\right)(\mathrm{CO})\left(\mathrm{L}^{57}\right)\right]$ & - & - & - & - & $\approx 25$ & - & - & - & - & - & - & - & - & - & {$[56]$} \\
\hline$\left[\mathrm{PPh}_{2}(\right.$ p-tolyl $\left.) \operatorname{Pd}\left(\mathbf{L}^{69}\right) \mathrm{Cl}\right]$ & $25.03(6.4)$ & $\begin{array}{c}26.9(4.1) \\
3.72\end{array}$ & $22.6(3.0)$ & $\begin{array}{c}18.4(2.8) \\
6.20\end{array}$ & 19.4(1.1) & - & - & - & - & - & - & $\begin{array}{c}- \\
5.46\end{array}$ & - & - & $\begin{array}{l}{[110]} \\
{[109]}\end{array}$ \\
\hline$\left[\mathrm{P}(\mathrm{p} \text {-tolyl })_{3} \mathrm{Pd}\left(\mathbf{L}^{69}\right) \mathrm{Cl}\right]$ & $32.9(1.8)$ & $\begin{array}{c}25.2(0.1) \\
5.18\end{array}$ & $36.9(5.1)$ & $\begin{array}{c}38.4(1.2) \\
5.29\end{array}$ & $29.5(2.0)$ & - & - & - & - & - & - & $\begin{array}{c}- \\
5.76 \\
\end{array}$ & - & - & $\begin{array}{l}{[110]} \\
{[109]}\end{array}$ \\
\hline$\left[\mathrm{PPh}_{2}(\right.$ p-tolyl $\left.) \mathrm{Pd}\left(\mathbf{L}^{76}\right) \mathrm{Cl}\right]$ & $5.6(0.4)$ & $0.01(0.005)$ & $0.1(0.2)$ & $1.3(2.2)$ & $2.8(1.2)$ & - & - & - & - & - & - & - & - & - & [110] \\
\hline$\left[\mathrm{P}(\mathrm{p} \text {-tolyl })_{3} \mathrm{Pd}\left(\mathrm{L}^{76}\right) \mathrm{Cl}\right]$ & $2.9(0.1)$ & $2.21(3.5)$ & $12.2(1.9)$ & $15.0(3.9)$ & 7.1(1.9) & - & - & - & - & - & - & - & - & - & [110] \\
\hline$\left[\mathrm{PPh}_{2}(\right.$ p-tolyl $\left.) \mathrm{Pd}\left(\mathbf{L}^{78}\right) \mathrm{Cl}\right]$ & $10.57(2.4)$ & $2.4(1.0)$ & $10(3)$ & $33.6(2.9)$ & $5.4(4.1)$ & - & - & - & - & - & - & - & - & - & [110] \\
\hline$\left[\mathrm{P}(\mathrm{p} \text {-tolyl })_{3} \mathrm{Pd}\left(\mathrm{L}^{78}\right) \mathrm{Cl}\right]$ & $10(2.4)$ & $3.02(0.3)$ & $2.8(0.4)$ & $3.9(0.024)$ & $7.0(3.6)$ & - & - & - & - & - & - & - & - & - & [110] \\
\hline$\left[\mathrm{PPh}_{2}(\mathrm{p}\right.$-tolyl $\left.) \mathrm{Pd}\left(\mathrm{L}^{67}\right) \mathrm{Cl}\right]$ & - & 4.51 & - & 5.11 & - & - & - & - & - & - & - & 4.83 & - & - & [109] \\
\hline$\left[\mathrm{P}(\mathrm{p} \text {-tolyl })_{3} \mathrm{Pd}\left(\mathrm{L}^{67}\right) \mathrm{Cl}\right]$ & - & 4.12 & - & 7.12 & - & - & - & - & - & - & - & 7.21 & - & - & [109] \\
\hline$\left[\mathrm{PPh}_{2}(\right.$ tert-But $\left.) \mathrm{Pd}\left(\mathrm{L}^{31}\right) \mathrm{Cl}\right]$ & - & - & - & - & - & - & - & - & - & - & - & - & 9.52 & - & [115] \\
\hline$\left[\mathrm{PPh}_{2}\left(\mathrm{CH}_{2} \mathrm{Ph}\right) \mathrm{Pd}\left(\mathrm{L}^{28}\right) \mathrm{Cl}\right]$ & - & - & - & - & - & - & - & - & - & - & - & - & 4.57 & - & [115] \\
\hline$\left[\mathrm{PPh}_{2}(\mathrm{Cl}) \mathrm{Pd}\left(\mathrm{L}^{28}\right) \mathrm{Cl}\right]$ & - & - & - & - & - & - & - & - & - & - & - & - & 21.7 & - & [115] \\
\hline$\left[\mathrm{PPh}_{3} \mathrm{Pd}\left(\mathbf{L}^{26}\right) \mathrm{Cl}\right]$ & - & - & - & - & - & - & - & - & - & - & - & - & 2.12 & - & [115] \\
\hline$\left[\mathrm{PPh}_{2}(\right.$ tert-But $\left.) \mathrm{Pd}\left(\mathrm{L}^{38}\right) \mathrm{Cl}\right]$ & - & - & - & - & - & - & - & - & - & - & - & - & - & $36.7(2.9)$ & [117] \\
\hline$\left[\mathrm{PPh}_{2}(\mathrm{nPr}) \mathrm{Pd}\left(\mathrm{L}^{26}\right) \mathrm{Cl}\right]$ & - & - & - & - & - & - & - & - & - & - & - & - & - & $>100$ & [117] \\
\hline$\left[\mathrm{PPh}_{3} \operatorname{Pd}\left(\mathbf{L}^{36}\right) \mathrm{Cl}\right]$ & - & - & - & - & - & - & - & - & - & - & - & - & - & $71.5(13.1)$ & [117] \\
\hline$\left[\mathrm{PPh}_{3} \mathrm{Pd}\left(\mathrm{L}^{35}\right) \mathrm{Cl}\right]$ & - & - & - & - & - & - & - & - & - & - & - & - & - & $>100$ & [117] \\
\hline$\left[\mathrm{PPh}_{3} \mathrm{Pd}\left(\mathbf{L}^{37}\right) \mathrm{Cl}\right]$ & - & - & - & - & - & - & - & - & - & - & - & - & - & $81.6(13.1)$ & [117] \\
\hline$\left[\mathrm{PPh}_{2}(\mathrm{p}\right.$-tolyl $\left.) \mathrm{Pd}\left(\mathrm{L}^{36}\right) \mathrm{Cl}\right]$ & - & - & - & - & - & - & - & - & - & - & - & - & - & $22.8(2.4)$ & [117] \\
\hline$\left[\mathrm{PPh}_{2}(\right.$ p-tolyl $\left.) \mathrm{Pd}\left(\mathbf{L}^{35}\right) \mathrm{Cl}\right]$ & - & - & - & - & - & - & - & - & - & - & - & - & - & $>100$ & [117] \\
\hline$\left[\mathrm{PPh}_{2}(\mathrm{p}\right.$-tolyl $\left.) \mathrm{Pd}\left(\mathbf{L}^{38}\right) \mathrm{Cl}\right]$ & - & - & - & - & - & - & - & - & - & - & - & - & - & $30.8(2.6)$ & [117] \\
\hline
\end{tabular}


Table 12. Cont.

\begin{tabular}{|c|c|c|c|c|c|c|c|c|c|c|c|c|c|c|c|}
\hline \multirow{2}{*}{ Complex $^{a}$} & \multicolumn{14}{|c|}{$\mathrm{IC}_{50} / \mu \mathrm{M}^{\mathrm{b}}$} & \multirow{2}{*}{ Ref. } \\
\hline & LU & MCF7 & Heap-1c1c7 & PC-3 & MDA-MB-231 & HepG2/CTR & HepG2/SB3 & HeLa & HL60 & Daudi & LoVo & A549 & HTB-81 & MRC-5 & \\
\hline$\left[\mathrm{PPh}_{2}\left(\mathrm{CH}_{2} \mathrm{Ph}\right) \mathrm{Pd}\left(\mathrm{L}^{38}\right) \mathrm{Cl}\right]$ & - & - & - & - & - & - & - & - & - & - & - & - & - & $>100$ & [117] \\
\hline$\left[\mathrm{PPh}_{3} \mathrm{Pd}\left(\mathbf{L}^{38}\right) \mathrm{Cl}\right]$ & - & - & - & - & - & - & - & - & - & - & - & - & - & $65.3(12.5)$ & [117] \\
\hline$\left[\mathrm{Pd}(3-\right.$ pic $\left.)\left(\mathrm{L}^{20}\right) \mathrm{Cl}\right]$ & - & - & - & - & - & - & - & $>100$ & $>100$ & $>100$ & $>100$ & - & - & - & [198] \\
\hline$\left[\mathrm{Pd}(2-\right.$ pic $\left.)\left(\mathrm{L}^{20}\right) \mathrm{Cl}\right]$ & - & - & - & - & - & - & - & $69.54(0.31)$ & $59.62(0.54)$ & 97.12(1.93) & $53.24(1.52)$ & - & - & - & [198] \\
\hline$\left[\mathrm{Pt}(3-\mathrm{pic})\left(\mathrm{L}^{20}\right) \mathrm{Cl}\right]$ & - & - & - & - & - & - & - & $98.90(2.33)$ & $>100$ & $>100$ & $>100$ & - & - & - & [198] \\
\hline$\left[\mathrm{Pt}(2-\mathrm{pic})\left(\mathrm{L}^{20}\right) \mathrm{Cl}\right]$ & - & - & - & - & - & - & - & $9.32(1.22)$ & $39.12(1.33)$ & $45.22(1.21)$ & $25.32(2.02)$ & - & - & - & [198] \\
\hline
\end{tabular}

${ }^{a} \mathrm{ClL}=3$-[( $p$-Chlorophenylimino)methyl]-4-cresol; pic = picoline. ${ }^{\mathrm{b}}$ By 3-(4,5-dimethylthiazol-2yl)-2,5-diphenyl-tetrazolium bromide (MTT) assay; Standard deviation given in parentheses. 


\subsubsection{Other Applications}

Besides their biological behavior, platinum-group dithiocarbamate complexes have potential utility as metal ion sensors [142,190], as single-source precursors for MOCVD [77], and as catalysts. Cyclometalated iridium(III) dithiocarbamate complexes of the type $\left[\operatorname{Ir}(\mathrm{dpci})_{2}\left(\mathrm{~S}_{2} \mathrm{CNR}_{2}\right)\right]$, where dpci $=3$,4-diphenylcinnoline; $\mathrm{NR}_{2}=\mathrm{NEt}_{2}\left(\mathbf{L}^{24}\right)$ and $\mathrm{N}$-carbazolyl $\left(\mathbf{L}^{\mathbf{8 2}}\right)$ are both highly selective and highly sensitive mercury chemosensors $[142,190]$. The sensors work by having a photoluminescent complex, with a relatively labile dithiocarbmate ligand, which can be scavenged by $\mathrm{Hg}^{2+}$ ions; the ideal scenario is that the presence of the $\mathrm{Hg}^{2+}$ ions act as a clear "on" switch, and these complexes display just such behavior. Furthermore, the complex $\left[\operatorname{Ir}(\mathrm{dpci})_{2}\left(\mathrm{~L}^{82}\right)\right]$ can operate as an AND logic gate, both changing color and displaying significant photophorescence in the presence of acetonitrile (which causes the color change) and $\mathrm{Hg}^{2+}$ ions [142].

Metal dithiocarbamate complexes are well-known as potential single-source precursors for the deposition of metal sulfide thin films [199,200]. The complexes [Pd(allyl) $\left.\left(\mathrm{S}_{2} \mathrm{CNR}_{2}\right)\right]$, with $\mathrm{NR}_{2}=\mathrm{NMe}_{2}\left(\mathbf{L}^{8}\right), \mathrm{NEt}_{2}\left(\mathbf{L}^{24}\right), \mathrm{N}(\mathrm{nPr})_{2}\left(\mathrm{~L}^{28}\right), \mathrm{N}(\mathrm{nBu})_{2}\left(\mathrm{~L}^{31}\right), \mathrm{N}\left(\mathrm{CH}_{2} \mathrm{Ph}\right)_{2}$ $\left(\mathbf{L}^{39}\right), \mathrm{N}(\mathrm{Me}) \mathrm{Bu}\left(\mathbf{L}^{11}\right), \mathrm{N}(\mathrm{Me}) \mathrm{Hex}\left(\mathbf{L}^{\mathbf{1 2}}\right)$, and $\mathrm{NC}_{5} \mathrm{H}_{10}\left(\mathbf{L}^{61}\right)$, may be used as single-source precursors for the MOCVD of $\mathrm{Pd}_{\mathrm{x}} \mathrm{S}_{\mathrm{y}}$ thin films. In particular, $\left[\mathrm{Pd}(\operatorname{allyl})\left(\mathbf{L}^{\mathbf{1 2}}\right)\right]$, was shown to produce films comprising $\mathrm{Pd}_{3} \mathrm{~S}, \mathrm{Pd}_{4} \mathrm{~S}, \mathrm{Pd}_{2.2} \mathrm{~S}, \mathrm{Pd}_{2.5} \mathrm{~S}$, and, predominately, $\mathrm{Pd}_{2.8} \mathrm{~S}$ at $350{ }^{\circ} \mathrm{C}$ [77]. The composition of such films may well be temperature-dependent [201]. Finally, in relation to complexes pertinent to this review, some ruthenium(II) dithiocarbamate complexes, namely $\left[\mathrm{Ru}(\mathrm{dppb})\left(\mathrm{S}_{2} \mathrm{CNR}_{2}\right)_{2}\right](\mathrm{dppb}=1,4-b i$ (diphenylphosphino)butane; $\mathrm{NR}_{2}=\mathrm{NEt}_{2}\left(\mathbf{L}^{24}\right), \mathrm{NC}_{5} \mathrm{H}_{10}\left(\mathbf{L}^{63}\right), \mathrm{N}\left(\mathrm{CH}_{2} \mathrm{CH}_{2}\right)_{2} \mathrm{O}\left(\mathbf{L}^{79}\right)$, and $\mathrm{N}($ cyclohexyl) $)\left(\mathbf{L}^{80}\right)$ have been demonstrated to be pre-catalysts for the epoxidation of cis-cyclooctene, with conversation rates of $c a 50-60 \%$, using $\mathrm{PhIO}$ as the oxidant [34]. The mechanism appears to involve loss of the dppb ligand to form a $\mathrm{Ru}=\mathrm{O}$ species. The dithiocarbamate ligands help to promote the displacement of $\mathrm{dppb}$ fostering the formation of the catalytic species, with both steric and electronic factors in play.

\section{Conclusions}

In summary, dithiocarbamates are highly versatile ligands, which are found in a wide variety of platinum group (and other) metal complexes. The resultant metal dithiocarbamate moiety may itself be the locus chemical or structural interest, or it may be that it is present in an ancillary capacity. The pendant organic groups on the dithiocarbamate nitrogen atom can be as varied as those on the parent amines, from which they are derived, allowing fine-tuning of both the electronic and steric environment around the ligand and hence of the structural and electronic properties of the complexes. The resultant platinum group metal complexes have been shown to have a variety of applications, with their anticancer properties being the most well studied. Comparison of X-ray structural data with NMR for these complexes indicates that the molecular structures are essentially the same in both the solid-state and in solution. Solution NMR, in particular, provides an excellent probe for metal-dithiocarbamate coordination, with the carbamoyl $\mathrm{C}$ atom and the $\alpha$-hydrogen atoms of the $\mathrm{N}$ substituents resonating at characteristic frequencies. Given their facile preparation, considerable stability and versatility in coordination chemistry, it is highly likely that the interest in these compounds will continue to grow.

Author Contributions: Writing—original draft preparation, review, and editing, Y.S.T.; Writingoriginal draft preparation, review, and editing, C.I.Y.; Writing—original draft preparation, review, and editing, E.R.T.T.; Conceptualization, writing—original draft preparation, review, and editing, P.J.H. All authors have read and agreed to the published version of the manuscript.

Funding: Research in dithiocarbamate chemistry at Sunway University is funded by Sunway University Sdn Bhd, grant number GRTIN-IRG-01-2021. The APC was funded by Sunway University Sdn Bhd.

Conflicts of Interest: The authors declare no conflict of interest. 


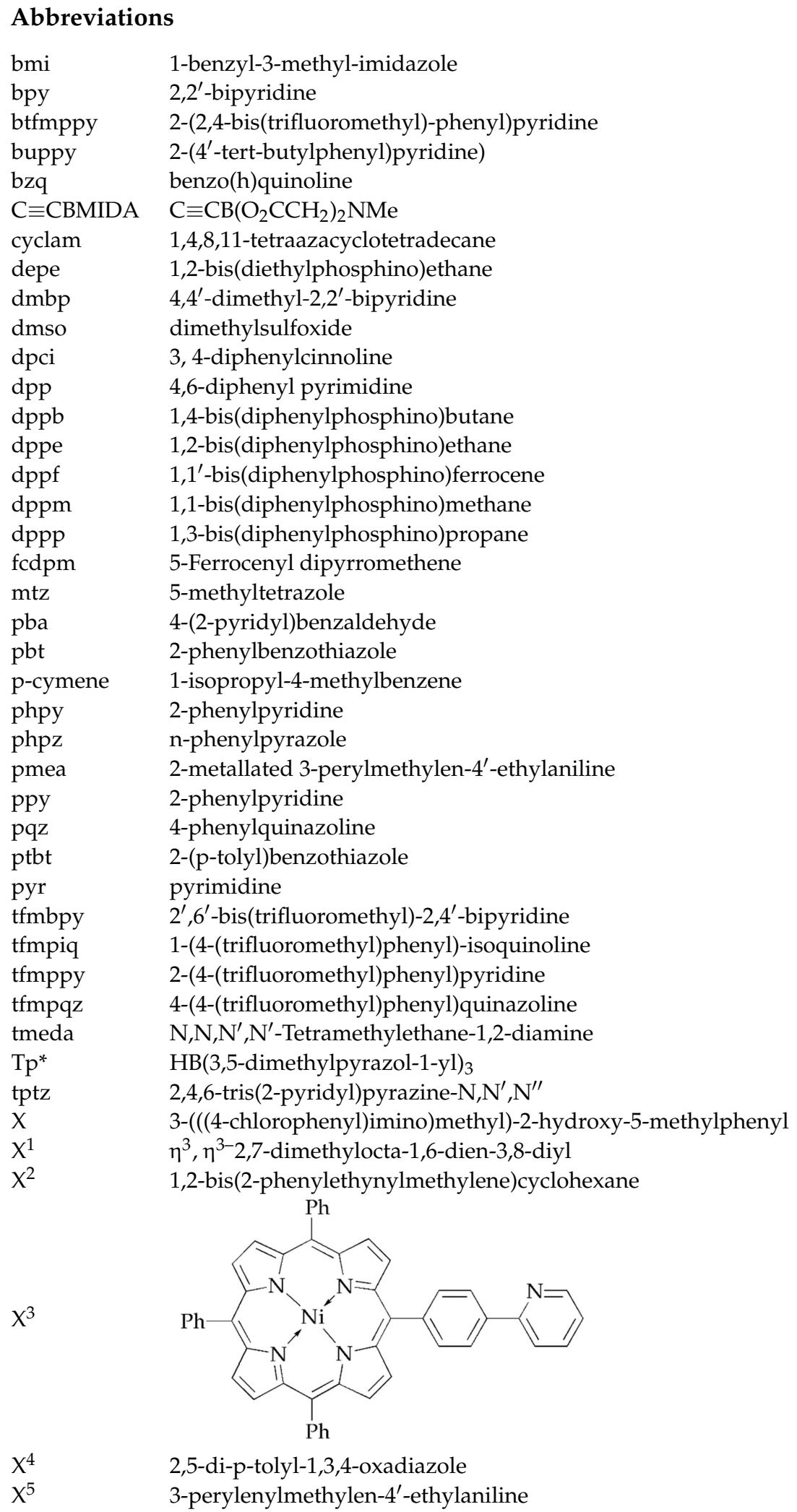

\section{References}

1. Coucouvanis, D. The chemistry of the dithioacid and 1,1-dithiolate complexes. In Progress in Inorganic Chemistry; Lippard, S.J., Ed.; John Wiley \& Sons: Hoboken, NJ, USA, 1970; Volume 11, pp. 234-371. [CrossRef]

2. Eisenberg, R. Structural systematics of 1,1- and 1,2-dithiolato chelates. In Progress in Inorganic Chemistry; Lippard, S.J., Ed.; John Wiley \& Sons: Hoboken, NJ, USA, 1970; Volume 12, pp. 295-369. [CrossRef] 
3. Coucouvanis, D. The chemistry of the dithioacid and 1,1-dithiolate complexes, 1968-1977. In Progress in Inorganic Chemistry; Lippard, S.J., Ed.; John Wiley \& Sons: Hoboken, NJ, USA, 1979; Volume 26, pp. 301-469. [CrossRef]

4. Hogarth, G. Transition metal dithiocarbamates: 1978-2003. In Progress in Inorganic Chemistry; Karlin, K.D., Ed.; John Wiley \& Sons: Hoboken, NJ, USA, 2005; Volume 53, pp. 71-561. [CrossRef]

5. Heard, P.J. Main group dithiocarbamate complexes. In Progress in Inorganic Chemistry; Karlin, K.D., Ed.; John Wiley \& Sons: Hoboken, NJ, USA, 2005; Volume 53, pp. 1-69. [CrossRef]

6. Tiekink, E.R.T. Aggregation patterns in the crystal structures of organometallic Group XV 1,1-dithiolates: The influence of the Lewis acidity of the central atom, metal- and ligand-bound steric bulk, and coordination potential of the 1,1-dithiolate ligands upon supramolecular architecture. CrystEngComm 2006, 8, 104-118. [CrossRef]

7. Tiekink, E.R.T.; Zukerman-Schpector, J. Stereochemical activity of lone pairs of electrons and supramolecular aggregation patterns based on secondary interactions involving tellurium in its 1,1-dithiolate structures. Coord. Chem. Rev. 2010, 254, 46-76. [CrossRef]

8. Tiekink, E.R.T. Exploring the topological landscape exhibited by binary zinc-triad 1,1-dithiolates. Crystals 2018, 8, 292. [CrossRef]

9. Tiekink, E.R.T. Perplexing coordination behaviour of potentially bridging bipyridyl-type ligands in the coordination chemistry of zinc and cadmium 1,1-dithiolate compounds. Crystals 2018, 8, 18. [CrossRef]

10. Lee, S.M.; Tiekink, E.R.T. A structural survey of poly-functional dithiocarbamate ligands and the aggregation patterns they sustain. Inorganics 2021, 9, 7. [CrossRef]

11. Tiekink, E.R.T. On the coordination role of pyridyl-nitrogen in the structural chemistry of pyridyl-substituted dithiocarbamate ligands. Crystals 2021, 11, 286. [CrossRef]

12. Masui, H. Metalloaromaticity. Coord. Chem. Rev. 2001, 219-221, 957-992. [CrossRef]

13. Tiekink, E.R.T.; Zukerman-Schpector, J. Emerging supramolecular synthons: C-H $\cdots \pi($ chelate) interactions in metal bis $(1,1-$ dithiolates). Chem. Commun. 2011, 47, 6623-6625. [CrossRef]

14. Tiekink, E.R.T. The remarkable propensity for the formation of $\mathrm{C}-\mathrm{H} \cdots \pi$ (chelate ring) interactions in the crystals of the firstrow transition metal dithiocarbamates and the supramolecular architectures they sustain. CrystEngComm 2020, 22, 7308-7733. [CrossRef]

15. Sredojević, D.; Bogdanović, G.A.; Tomić, Z.D.; Zarić, S.D. Stacking vs. CH- $\pi$ interactions between chelate and aryl rings in crystal structures of square-planar transition metal complexes. CrystEngComm 2007, 9, 793-798. [CrossRef]

16. Tan, Y.S.; Halim, S.N.A.; Molloy, K.C.; Sudlow, A.L.; Otero-de-la-Roza, A.; Tiekink, E.R.T. Persistence of C-H $\cdots \pi($ chelate ring) interactions in the crystal structures of $\mathrm{Pd}\left(\mathrm{S}_{2} \mathrm{COR}\right)_{2}$. The utility of $\mathrm{Pd}\left(\mathrm{S}_{2} \mathrm{COR}\right)_{2}$ as precursors for palladium sulphide materials. CrystEngComm 2016, 18, 1105-1117. [CrossRef]

17. Tiekink, E.R.T. Supramolecular assembly based on "emerging" intermolecular interactions of particular interest to coordination chemists. Coord. Chem. Rev. 2017, 345, 209-228. [CrossRef]

18. Shinde, S.D.; Sakla, A.P.; Shankaraiah, N. An insight into medicinal attributes of dithiocarbamates: Bird's eye view. Bioorg. Chem. 2020, 105, 104346. [CrossRef] [PubMed]

19. Buac, D.; Schmitt, S.; Ventro, G.; Kona, F.R.; Dou, Q.P. Dithiocarbamate-based coordination compounds as potent proteasome inhibitors in human cancer cells. Mini Rev. Med. Chem. 2012, 12, 1193-1201. [CrossRef]

20. Nagy, E.M.; Ronconi, L.; Nardon, C.; Fregona, D. Noble metal-dithiocarbamates precious allies in the fight against cancer. Mini Rev. Med. Chem. 2012, 12, 1216-1229. [CrossRef]

21. Hogarth, G. Metal-dithiocarbamate complexes: Chemistry and biological activity. Mini Rev. Med. Chem. 2012, 12, 1202-1215. [CrossRef]

22. Ahmed, A.J. Metal complexes of dithiocarbamate derivatives and its biological activity. Asian J. Chem. 2018, 30, 2595-2602. [CrossRef]

23. Sarker, J.C.; Hogarth, G. Dithiocarbamate complexes as single source precursors to nanoscale binary, ternary and quaternary metal sulfides. Chem. Rev. 2021, 121, 6057-6123. [CrossRef] [PubMed]

24. Lewis, E.; Haigh, S.; O'Brien, P. The synthesis of metallic and semiconducting nanoparticles from reactive melts of precursors. J. Mater. Chem. A 2014, 2, 570-580. [CrossRef]

25. Nagy, M.; Nardon, C.; Giovagnini, L.; Marchiò, L.; Trevisan, A.; Fregona, D. Promising anticancer mono- and dinuclear ruthenium(III) dithiocarbamato complexes: Systematic solution studies. Dalton Trans. 2011, 40, 11885-11895. [CrossRef] [PubMed]

26. Almazahreh, L.; El-khateeb, M.; Harb, M.; Görls, H.; Weigand, W. Synthesis and characterization of platinum and palladium pyrrolidinedithiocarbamate complexes. Transit. Met. Chem. 2013, 38, 377-383. [CrossRef]

27. Mei, Q.; Shi, Y.; Hua, Q.; Tong, B. Phosphorescent chemosensor for $\mathrm{Hg}^{2+}$ based on an iridium(III) complex coordinated with 4-phenylquinazoline and carbazole dithiocarbamate. RSC Adv. 2015, 5, 74924-74931. [CrossRef]

28. Batsanov, A.S.; Churakov, A.V.; Howard, J.A.K.; Hughes, A.K.; Johnson, A.L.; Kingsley, A.J.; Neretin, I.S.; Wade, K. Transition metal dicarbollide complexes: Synthesis, molecular, crystal and electronic structures of $\left[\mathrm{M}\left(\mathrm{C}_{2} \mathrm{~B}_{9} \mathrm{H}_{11}\right)\left(\mathrm{NMe}_{2}\right)_{3}\right](\mathrm{M}=\mathrm{Nb}$ or Ta) and their insertion reactions with $\mathrm{CO}_{2}$ and $\mathrm{CS}_{2}$. J. Chem. Soc. Dalton Trans. 1999, 3867-3875. [CrossRef]

29. Taylor, R.; Wood, P.A. A million crystal structures: The whole is greater than the sum of its parts. Chem. Rev. 2019, 119, 9427-9477. [CrossRef] 
30. Bruno, I.J.; Cole, J.C.; Edgington, P.R.; Kessler, M.; Macrae, C.F.; McCabe, P.; Pearson, J.; Taylor, R. New software for searching the Cambridge Structural Database and visualizing crystal structures. Acta Crystallogr. Sect. B Struct. Sci. Cryst. Eng. Mater. 2002, 58, 389-397. [CrossRef]

31. Brandenburg, K.; Berndt, M. DIAMOND, Version 3.2k; GbR: Bonn, Germany, 2006.

32. Nagy, E.M.; Pettenuzzo, A.; Boscutti, G.; Marchi, L.; Via, L.D.; Fregona, D. Ruthenium(II/III)-based compounds with encouraging antiproliferative activity against non-small-cell lung cancer. Chem. Eur. J. 2012, 18, 14464-14472. [CrossRef] [PubMed]

33. Wu, F.-H.; Liu, Y.-L.; Duan, T.; Lu, L.; Zhang, Q.-F.; Leung, W.-H. Synthesis, structure and electrochemistry of $\left[\mathrm{Ru}_{2}\left(\mathrm{~S}_{2} \mathrm{CNMe}\right)_{3}\left(\mu_{3}-\right.\right.$ $\left.\left.\mathrm{S}_{2} \mathrm{CNMe}_{2}\right)_{2}\right]_{2}\left[\mathrm{Mo}_{6} \mathrm{O}_{19}\right] \cdot 2 \mathrm{CH}_{3} \mathrm{COCH}_{3}$. Z. Naturforsch. B Chem. Sci. 2009, 64, 800-804. [CrossRef]

34. Santos, K.; Dinelli, L.R.; Bogado, A.L.; Ramos, L.A.; Cavalheiro, É.T.; Ellena, J.; Castellano, E.E.; Batista, A.A. Crystal structure and catalytic activity of ruthenium (II)/dithiocarbamate complexes in the epoxidation of cyclooctene. Inorg. Chim. Acta 2015, 429, 237-242. [CrossRef]

35. Pinheiro, S.O.; de Sousa, J.R.; Santiago, M.O.; Carvalho, I.M.M.; Silva, A.L.R.; Batista, A.A.; Castellano, E.E.; Ellena, J.; Moreira, I.S.; Diogenes, I.C.N. Synthesis, characterization and structure of ruthenium(II) phosphine complexes with N-heterocyclic thiolate ligands. Inorg. Chim. Acta 2006, 359, 391-400. [CrossRef]

36. Ng, S.; Ziller, J.W.; Farmer, P.J. Multiple pathways for the oxygenation of a ruthenium(II) dithiocarbamate complex: S-oxygenation and S-extrusion. Inorg. Chem. 2004, 43, 8301-8309. [CrossRef] [PubMed]

37. Cookson, J.; Vickers, M.S.; Paul, R.L.; Cowley, A.R.; Beer, P.D. Amide functionalised dithiocarbamate ruthenium(II) bis-bipyridyl receptors: A new class of redox-responsive anion sensor. Inorg. Chim. Acta 2008, 361, 1689-1698. [CrossRef]

38. Milsmann, C.; Bill, E.; Weyhermuller, T.; George, S.D.; Wieghardt, K. Electronic structures of $\left[\mathrm{Ru}^{\mathrm{II}}(\mathrm{cyclam})\left(\mathrm{Et}_{2} \mathrm{dtc}\right)\right]^{+}$, $[\mathrm{Ru}(\mathrm{cyclam})(\mathrm{tdt})]^{+}$, and $[\mathrm{Ru}(\mathrm{cyclam})(\mathrm{tdt})]^{2+}:$ An X-ray absorption spectroscopic and computational study $(\mathrm{tdt}=$ toluene-3,4dithiolate; $\mathrm{Et}_{2} \mathrm{dtc}=\mathrm{N}, \mathrm{N}$-diethyldithiocarbamate(1-)). Inorg. Chem. 2009, 48, 9754-9766. [CrossRef] [PubMed]

39. Hurtubise, V.L.; McArdle, J.M.; Naeem, S.; Toscani, A.; White, A.J.P.; Long, N.J.; Wilton-Ely, J.D.E.T. Multimetallic complexes and functionalized nanoparticles based on unsymmetrical dithiocarbamate ligands with allyl and propargyl functionality. Inorg. Chem. 2014, 53, 11740-11748. [CrossRef] [PubMed]

40. Naeem, S.; Ogilvie, E.; White, A.J.P.; Hogarth, G.; Wilton-Ely, J.D.E.T. The functionalisation of ruthenium(II) and osmium(II) alkenyl complexes with amine- and alkoxy-terminated dithiocarbamates. Dalton Trans. 2010, 39, 4080-4089. [CrossRef] [PubMed]

41. Wilton-Ely, J.D.E.T.; Solanki, D.; Hogarth, G. Multifunctional dithiocarbamates as ligands towards the rational synthesis of polymetallic arrays: An example based on a piperizine-derived dithiocarbamate ligand. Eur. J. Inorg. Chem. 2005, 2005, 4027-4030. [CrossRef]

42. Knight, E.J.; Cowley, A.R.; Hogarth, G.; Wilton-Ely, J.D.E.T. Bifunctional dithiocarbamates: A bridge between coordination chemistry and nanoscale materials. Dalton Trans. 2009, 607-609. [CrossRef]

43. Wu, F.-H.; Duan, T.; Lu, L.; Zhang, Q.-F.; Leung, W.-H. Synthesis and reactivity of ruthenium complexes with 1,1'-dithiolate ligands. J. Organomet. Chem. 2009, 694, 3844-3851. [CrossRef]

44. Wu, F.-H.; Duan, T.-K.; Lu, L.-D.; Zhang, Q.-F. Syntheses and structures of two ruthenium(III) $\operatorname{complexes}\left[\mathrm{Ru}\left(\mathrm{PPh}_{3}\right)_{2}\left(\mathrm{~S}_{2} \mathrm{CNR}_{2}\right) \mathrm{Cl}_{2}\right]$ . $\mathrm{CH}_{2} \mathrm{Cl}_{2}(\mathrm{R}=\mathrm{Et}$ and $i \mathrm{Pr})$. Chin. J. Struct. Chem. 2010, 29, 23-28.

45. Sharma, S.; Chandra, M.; Pandey, D.S. New multifunctional complexes $\left[\mathrm{Ru}\left(\kappa^{3}-\mathrm{L}\right)\left(\mathrm{EPh}_{3}\right)_{2} \mathrm{Cl}\right]^{+}[\mathrm{E}=\mathrm{P}, \mathrm{As} ; \mathrm{L}=2,4,6$-Tris(2-pyridyl)1,3,5-triazine] containing both group V and polypyridyl ligands. Eur. J. Inorg. Chem. 2004, 2004, 3555-3563. [CrossRef]

46. Deeming, A.J.; Forth, C.; Hogarth, G. Reactions of trans- $\left[\mathrm{RuCl}_{2}(\mathrm{CO})_{2}\left(\mathrm{PEt}_{3}\right)_{2}\right]$ with 1,1-dithiolates: Stepwise formation of cis- $\left[\mathrm{Ru}(\mathrm{CO})\left(\mathrm{PEt}_{3}\right)\left(\mathrm{S}_{2} \mathrm{X}\right)\right]\left(\mathrm{X}=\mathrm{CNMe}_{2}, \mathrm{CNEt}_{2}, \mathrm{COEt}, \mathrm{P}(\mathrm{OEt})_{2}, \mathrm{PPh}_{2}\right)$. J. Organomet. Chem. 2006, 691, 79-85. [CrossRef]

47. Boyd, P.D.W.; Hart, M.C.; Pritzwald-Stegmann, J.R.F.; Roper, W.R.; Wright, L.J. Selective substitution of one of the substituents on germanium in coordinatively unsaturated ruthenium germyl complexes. Organometallics 2012, 31, 2914-2921. [CrossRef]

48. Kwok, W.-H.; Lu, G.-L.; Rickard, C.E.F.; Roper, W.R.; Wright, L.J. Tethered silyl complexes from nucleophilic substitution reactions at the $\mathrm{Si}-\mathrm{Cl}$ bond of the chloro(diphenyl)silyl ligand in $\mathrm{Ru}(\mathrm{SiClPh} 2)\left(\kappa^{2}-\mathrm{S}_{2} \mathrm{CNMe}\right)(\mathrm{CO})\left(\mathrm{PPh}_{3}\right)_{2}$. J. Organomet. Chem. 2004, 689, 2979-2987. [CrossRef]

49. McQueen, C.M.A.; Hill, A.F.; Sharma, M.; Singh, S.K.; Ward, J.S.; Willis, A.C.; Young, R.D. Synthesis and reactivity of osmium and ruthenium PBP-LXL boryl pincer complexes. Polyhedron 2016, 120, 185-195. [CrossRef]

50. Wang, X.-Y.; Shi, H.-T.; Zhang, Q.-F. Synthesis and structure of a ruthenium(II)-dithiocarbamate $\operatorname{complex}\left[\mathrm{RuH}(\mathrm{CO})(\mathrm{PPh})_{2}(4-\right.$ CIPhNHCS 2$)] \cdot \mathrm{C}_{6} \mathrm{H}_{14}$. Chin. J. Struct. Chem. 2011, 30, 470-473.

51. Toscani, A.; Heliövaara, E.K.; Hena, J.B.; White, A.J.P.; Wilton-Ely, J.D.E.T. Multimetallic alkenyl complexes bearing macrocyclic dithiocarbamate ligands. Organometallics 2015, 34, 494-505. [CrossRef]

52. Hill, A.F.; Stewart, C.D.; Ward, J.S. Organometallic chemistry of ethynyl boronic acid MIDA ester, HC [triple bond, length as m-dash] CB (O 2 CCH 2) 2 NMe. Dalton Trans. 2015, 44, 5713-5726. [CrossRef] [PubMed]

53. Naeem, S.; White, A.J.P.; Hogarth, G.; Wilton-Ely, J.D.E.T. Multifunctional dithiocarbamates: Synthesis and ring-closing metathesis of diallyldithiocarbamate complexes. Organometallics 2010, 29, 2547-2556. [CrossRef]

54. Bartlett, M.J.; Frogley, B.J.; Hill, A.F.; Sharma, M.; Smith, M.K.; Ward, J.S. Hydrogenating an organometallic carbon chain: Buten-yn-diyl ( $\mathrm{CH}=\mathrm{CHC} \equiv \mathrm{C})$ as a missing link. Dalton Trans. 2019, 48, 16534-16554. [CrossRef] [PubMed]

55. Macgregor, M.J.; Hogarth, G.; Thompson, A.L.; Wilton-Ely, J.D.E.T. Multimetallic arrays: Symmetrical and unsymmetrical bi-, tri-, and tetrametallic organometallic complexes of ruthenium(II) and osmium(II). Organometallics 2009, 28, 197-208. [CrossRef] 
56. Ghosh, M.K.; Mandal, S.; Mohapatra, S.; Chatterjee, A.; Bhattacharyya, A.; Chattopadhyay, S. Spectroscopic, structural, electrochemical, and cytotoxicity studies on dithiocarbamato-chelated ruthenium organometallics incorporating imine-phenol function. J. Coord. Chem. 2019, 72, 180-200. [CrossRef]

57. Ollivier, A.; Foresti, R.; El Ali, Z.; Martens, T.; Kitagishi, H.; Motterlini, R.; Rivard, M. Design and biological evaluation of manganese- and ruthenium-based hybrid CO-RMs (HYCOs). ChemMedChem 2019, 14, 1684-1691. [CrossRef] [PubMed]

58. Hogarth, G.; Faulkner, S. Cyclopentadienyl ruthenium(II) dithiocarbamate complexes: Crystal structures of [CpRu(PPh 3 ) $\left.\left(\mathrm{S}_{2} \mathrm{CNPr}_{2}\right)\right]$ and $\left[\mathrm{CpRu}\left(\mathrm{PPh}_{3}\right)\left(\mathrm{S}_{2} \mathrm{CNMeBu}\right)\right]$. Inorg. Chim. Acta 2006, 359, 1018-1022. [CrossRef]

59. Lalrempuia, R.; Suante, H.; Yennawar, H.P.; Kollipara, M.R. Reactivity of the $\left[\mathrm{CpM}\left(\mathrm{PPh}_{3}\right)_{2}\right]^{+}(\mathrm{M}=\mathrm{Ru}, \mathrm{Os})$ fragment with diethyldithiocarbamate. Polyhedron 2007, 26, 867-870. [CrossRef]

60. El-Khateeb, M.; Al-Noaimi, M.; Harb, M.; Gorls, H.; Weigand, W. Half-sandwich ruthenium complexes of heterocyclicdithiocarboxylato ligands. Jordan J. Chem. 2008, 3, 147.

61. Lu, X.L.; Vittal, J.J.; Tiekink, E.R.T.; Tan, G.K.; Kuan, S.L.; Goh, L.Y.; Hor, T.S.A. Comparative reactivity studies of dppf-containing $\mathrm{CpRu}^{\mathrm{II}}$ and $\left(\mathrm{C}_{6} \mathrm{Me}_{6}\right) \mathrm{Ru}^{\mathrm{II}}$ complexes towards different donor ligands (dppf=1,1'-bis(diphenylphosphino)ferrocene). J. Organomet. Chem. 2004, 689, 1978-1990. [CrossRef]

62. Rheingold, A.L.; Bitterwolf, T.E. Carbonyl-cyclopentadienyl-(N,N-dimethyldithiocarbamato)-ruthenium. CSD Commun. 2019. [CrossRef]

63. Cheung, W.-M.; Zhang, Q.-F.; Wiliams, I.D.; Leung, W.-H. An unsaturated half-sandwich ruthenium(II) complex containing a dithioimidodiphosphinate ligand. Inorg. Chim. Acta 2006, 359, 782-788. [CrossRef]

64. Tay, E.P.L.; Kuan, S.L.; Leong, W.K.; Goh, L.Y. Synthetic and X-ray structural and reactivity studies of $\mathrm{Cp}^{*} \mathrm{Ru} \mathrm{u}^{\mathrm{IV}}$ complexes containing bidentate dithiocarbonate, xanthate, carbonate, and phosphinate ligands $\left(C \mathrm{p}^{*}=\eta^{5}-\mathrm{C}_{5} \mathrm{Me}_{5}\right)$. Inorg. Chem. 2007, 46, 1440-1450. [CrossRef]

65. Kuan, S.L.; Tay, E.P.L.; Leong, W.K.; Goh, L.Y.; Lin, C.Y.; Gill, P.M.W.; Webster, R.D. Highly oxidized ruthenium organometallic compounds. The synthesis and one-electron electrochemical oxidation of $\left[\mathrm{Cp}^{*} \mathrm{Ru}^{\mathrm{IV}} \mathrm{Cl}_{2}\left(\mathrm{~S}_{2} \mathrm{CR}\right)\right]\left(\mathrm{Cp} \mathrm{p}^{*}=\eta^{5}-\mathrm{C}_{5} \mathrm{Me}_{5}, \mathrm{R}=\mathrm{NMe}_{2}, \mathrm{NEt}_{2}\right.$, $\left.\mathrm{O}^{i} \mathrm{Pr}\right)$. Organometallics 2006, 25, 6134-6141. [CrossRef]

66. Xu, C.; Pullarkat, S.A.; Goh, L.Y. Bis(allyl)ruthenium(iv)-initiated S-S and C-S bond cleavages in tetraalkylthiuram sulfides. Formation and X-ray crystal structures of dithiocarbamato complexes. Aust. J. Chem. 2009, 62, 1537-1543. [CrossRef]

67. Dou, Y.-H.; Xu, S.-D.; Chen, Y.; Wu, X.-H. Synthesis, characterization, and anticancer activity of dithiocarbamate ruthenium(II) complexes. Phosphorus Sulfur Silicon Relat. Elem. 2017, 192, 1219-1223. [CrossRef]

68. Seino, H.; Yoshikawa, T.; Hidai, M.; Mizobe, Y. Preparation of mononuclear and dinuclear Rh hydrotris(pyrazolyl)borato complexes containing arenethiolato ligands and conversion of the mononuclear complexes into dinuclear $\mathrm{Rh}-\mathrm{Rh}$ and $\mathrm{Rh}-\mathrm{Ir}$ complexes with bridging arenethiolato ligands. Dalton Trans. 2004, 3593-3600. [CrossRef] [PubMed]

69. Dixon, L.S.H.; Hill, A.F.; Sinha, A.; Ward, J.S. N-heterocyclic silyl pincer ligands. Organometallics 2014, 33, 653-658. [CrossRef]

70. Mansouri, G.; Heidarizadi, F.; Naghipour, A.; Notash, B. Synthesis, characterization and antibacterial study of cyclometalated rhodium(III) complex containing dithiocarbamate. J. Mol. Struct. 2016, 1121, 128-134. [CrossRef]

71. Mukherjee, T.; Sen, B.; Patra, A.; Banerjee, S.; Hundal, G.; Chattopadhyay, P. Cyclometalated rhodium(III) complexes bearing dithiocarbamate derivative: Synthesis, characterization, interaction with DNA and biological study. Polyhedron 2014, 69, 127-134. [CrossRef]

72. Steffen, A.; Tay, M.G.; Batsanov, A.S.; Howard, J.A.K.; Beeby, A.; Vuong, K.Q.; Sun, X.-Z.; George, M.W.; Marder, T.B. 2,5-Bis(p-Rarylethynyl)rhodacyclopentadienes show intense fluorescence: Denying the presence of a heavy atom. Angew. Chem. Int. Ed. 2010, 49, 2349-2353. [CrossRef] [PubMed]

73. Bobinihi, F.F.; Onwudiwe, D.C.; Ekennia, A.C.; Okpareke, O.C.; Arderne, C.; Lane, J.R. Group 10 metal complexes of dithiocarbamates derived from primary anilines: Synthesis, characterization, computational and antimicrobial studies. Polyhedron 2019, 158, 296-310. [CrossRef]

74. Poirier, S.; Tailleur, E.; Lynn, H.; Reber, C. Characterization of Pd - H H-C interactions in bis-dimethyldithiocarbamate palladium(II) and its deuterated analog by luminescence spectroscopy at variable pressure. Dalton Trans. 2016, 45, 10883-10886. [CrossRef]

75. Poirier, S.; Lynn, H.; Reber, C.; Tailleur, E.; Marchivie, M.; Guionneau, P.; Probert, M.R. Variation of M-.H-C interactions in square-planar complexes of nickel(II), palladium(II), and platinum(II) probed by luminescence spectroscopy and X-ray diffraction at variable pressure. Inorg. Chem. 2018, 57, 7713-7723. [CrossRef]

76. Poirier, S.; Guionneau, P.; Luneau, D.; Reber, C. Why do the luminescence maxima of isostructural palladium(II) and platinum(II) complexes shift in opposite directions? Can. J. Chem. 2014, 92, 958-965. [CrossRef]

77. Birri, A.; Harvey, B.; Hogarth, G.; Subasi, E.; Ugur, F. Allyl palladium dithiocarbamates and related dithiolate complexes as precursors to palladium sulfides. J. Organomet. Chem. 2007, 692, 2448-2455. [CrossRef]

78. Shaheen, F.; Badshah, A.; Gielen, M.; Dusek, M.; Fejfarova, K.; de Vos, D.; Mirza, B. Synthesis, characterization, antibacterial and cytotoxic activity of new palladium(II) complexes with dithiocarbamate ligands: X-ray structure of bis(dibenzyl-1-S:S'dithiocarbamato)Pd(II). J. Organomet. Chem. 2007, 692, 3019-3026. [CrossRef]

79. Ehsan, M.A.; Ming, H.N.; McKee, V.; Peiris, T.A.N.; Wijayantha-Kahagala-Gamage, U.; Arifin, Z.; Mazhar, M. Vysotskite structured photoactive palladium sulphide thin films from dithiocarbamate derivatives. New J. Chem. 2014, 38, 4083-4091. [CrossRef]

80. Ferreira, I.P.; de Lima, G.M.; Paniago, E.B.; Takahashi, J.A.; Pinheiro, C.B. Synthesis, characterization and antifungal activity of new dithiocarbamate-based complexes of $\mathrm{Ni}(\mathrm{II}), \mathrm{Pd}(\mathrm{II})$ and $\mathrm{Pt}(\mathrm{II})$. Inorg. Chim. Acta 2014, 423, 443-449. [CrossRef] 
81. Phadnis, P.P.; Jain, V.K.; Schurr, T.; Klein, A.; Lissner, F.; Schleid, T.; Kaim, W. Synthesis, spectroscopy, structure and photophysical properties of dinaphthylmethylarsine complexes of palladium(II) and platinum(II). Inorg. Chim. Acta 2005, 358, $2609-2617$. [CrossRef]

82. Bobinihi, F.F.; Onwudiwe, D.C.; Hosten, E.C. Synthesis and characterization of homoleptic group 10 dithiocarbamate complexes and heteroleptic $\mathrm{Ni}$ (II) complexes, and the use of the homoleptic $\mathrm{Ni}$ (II) for the preparation of nickel sulphide nanoparticles. J. Mol. Struct. 2018, 1164, 475-485. [CrossRef]

83. Hogarth, G.; Rainford-Brent, E.-J.C.-R.C.R.; Kabir, S.E.; Richards, I.; Wilton-Ely, J.D.E.T.; Zhang, Q. Functionalised dithiocarbamate complexes: Synthesis and molecular structures of 2-diethylaminoethyl and 3-dimethylaminopropyl dithiocarbamate complexes $\left[\mathrm{M}\left\{\mathrm{S}_{2} \mathrm{CN}\left(\mathrm{CH}_{2} \mathrm{CH}_{2} \mathrm{NEt}_{2}\right)_{2}\right\}_{\mathrm{n}}\right]$ and $\left[\mathrm{M}\left\{\mathrm{S}_{2} \mathrm{CN}\left(\mathrm{CH}_{2} \mathrm{CH}_{2} \mathrm{CH}_{2} \mathrm{NMe}_{2}\right)_{2}\right\}_{\mathrm{n}}\right](\mathrm{n}=2, \mathrm{M}=\mathrm{Ni}, \mathrm{Cu}, \mathrm{Zn}, \mathrm{Pd} ; \mathrm{n}=3, \mathrm{M}=\mathrm{Co})$. Inorg. Chim. Acta 2009, 362, 2020-2026. [CrossRef]

84. Onwudiwe, D.C.; Ekennia, A.C.; Mogwase, B.M.S.; Olubiyi, O.O.; Hosten, E. Palladium(II) and platinum(II) complexes of N-butylN-phenyldithiocarbamate: Synthesis, characterization, biological activities and molecular docking studies. Inorg. Chim. Acta 2016, 450, 69-80. [CrossRef]

85. Konarev, D.V.; Kovalevsky, A.Y.; Otsuka, A.; Saito, G.; Lyubovskaya, R.N. Neutral and ionic complexes of $\mathrm{C}_{60}$ with metal dibenzyldithiocarbamates. Reversible dimerization of $\mathrm{C}_{60}{ }^{\bullet-}$ in ionic multicomponent complex $\left[\mathrm{Cr}^{\mathrm{I}}\left(\mathrm{C}_{6} \mathrm{H}_{6}\right)_{2}{ }^{\bullet+}\right] \cdot\left(\mathrm{C}_{60}{ }^{\bullet-}\right) \cdot 0.5\left[\mathrm{Pd}(\mathrm{dbdtc})_{2}\right]$. Inorg. Chem. 2005, 44, 9547-9553. [CrossRef]

86. Gupta, A.N.; Kumar, V.; Singh, V.; Manar, K.K.; Drew, M.G.B.; Singh, N. Intermolecular anagostic interactions in group 10 metal dithiocarbamates. CrystEngComm 2014, 16, 9299-9307. [CrossRef]

87. Yadav, M.K.; Rajput, G.; Prasad, L.B.; Drew, M.G.B.; Singh, N. Rare intermolecular M $\cdots H-C$ anagostic interactions in homoleptic $\mathrm{Ni}(\mathrm{II})-\mathrm{Pd}(\mathrm{II})$ dithiocarbamate complexes. New J. Chem. 2015, 39, 5493-5499. [CrossRef]

88. Genre, C.; Levasseur-Theriault, G.; Reber, C. Emitting-state properties of square-planar dithiocarbamate complexes of palladium(II) and platinum(II) probed by pressure-dependent luminescence spectroscopy. Can. J. Chem. 2009, 87, 1625-1635. [CrossRef]

89. Shaheen, F.; Badshah, A.; Anjum, S.; Saqib, A. Bis(piperidine-1-dithiocarbamato- ${ }^{2} S, S^{\prime}$ )palladium(II). Acta Crystallogr. Sect. E Struct. Rep. Online 2006, 62, m329-m330. [CrossRef]

90. Shahzadi, S.; Ali, S.; Badshah, A.; Shaheen, F.; Ahmed, F.; Fettouhi, M. Synthesis and crystal structure of bis(4-methylpiperidinedithiocarbamato-S,S')-palladium(II). J. Chem. Cryst. 2006, 36, 567-570. [CrossRef]

91. Mukherjee, T.; Sarkar, S.; Marek, J.; Zangrando, E.; Chattopadhyay, P. Palladium(II) complexes of dithiocarbamic acids: Synthesis, characterization, crystal structure and DNA binding study. Transit. Met. Chem. 2012, 37, 155-161. [CrossRef]

92. Torres-Huerta, A.; Höpfl, H.; Tlahuext, H.; Hernández-Ahuactzi, I.F.; Sánchez, M.; Reyes-Martínez, R.; Morales-Morales, D. Dinuclear macrocyclic palladium dithiocarbamates derived from the homologous series of aliphatic $1, x$-diamines $(x=4-10)$. Eur. J. Inorg. Chem. 2013, 2013, 61-69. [CrossRef]

93. Webber, P.R.A.; Drew, M.G.B.; Hibbert, R.; Beer, P.D. Transition metal-directed self-assembly of calix[4]arene based dithiocarbamate ligands. Dalton Trans. 2004, 1127-1135. [CrossRef]

94. Sherwood, R.; de Rivera, F.G.; Wan, J.H.; Zhang, Q.; White, A.J.P.; Rossell, O.; Hogarth, G.; Wilton-Ely, J.D.E.T. Multimetallic complexes based on a diphosphine-dithiocarbamate "janus" ligand. Inorg. Chem. 2015, 54, 4222-4230. [CrossRef]

95. Gupta, R.K.; Pandey, R.; Singh, R.; Srivastava, N.; Maiti, B.; Saha, S.; Li, P.; Xu, Q.; Pandey, D.S. Heteroleptic dipyrrinato complexes containing 5-ferrocenyldipyrromethene and dithiocarbamates as coligands: Selective chromogenic and redox probes. Inorg. Chem. 2012, 51, 8916-8930. [CrossRef]

96. Singh, S.K.; Kumar, V.; Drew, M.G.B.; Singh, N. Syntheses, crystal structures and photoluminescent properties of new heteroleptic $\mathrm{Ni}(\mathrm{II})$ and $\mathrm{Pd}(\mathrm{II})$ complexes of ferrocene functionalized dithiocarbamate-and dipyrromethene ligands. Inorg. Chem. Commun. 2013, 37, 151-154. [CrossRef]

97. Li, H.; Jiang, X.-F.; Yu, S.-Y. Syntheses, characterization and crystal structures of dithiocarbamate-based mononuclear palladium(II) complexes. Chin. J. Struct. Chem. 2017, 36, 1185-1192. [CrossRef]

98. Wiench, J.W.; Michon, C.; Ellern, A.; Hazendonk, P.; Iuga, A.; Angelici, R.J.; Pruski, M. Solid-state NMR investigations of the immobilization of $\mathrm{a} \mathrm{BF}_{4}{ }^{-}$salt of a palladium(II) complex on silica. J. Am. Chem. Soc. 2009, 131, 11801-11810. [CrossRef] [PubMed]

99. Xu, S.D.; Wu, X.H. Bimetallic DppfM(II) (M=Pt and Pd) dithiocarbamate complexes: Synthesis, characterization, and anticancer activity. J. Chem. Res. 2019, 43, 437-442. [CrossRef]

100. Jantan, K.A.; Kwok, C.Y.; Chan, K.W.; Marchiò, L.; White, A.J.P.; Deplano, P.; Serpe, A.; Wilton-Ely, J.D.E.T. From recovered metal waste to high-performance palladium catalysts. Green Chem. 2017, 19, 5846-5853. [CrossRef]

101. Knight, E.R.; Leung, N.H.; Lin, Y.H.; Cowley, A.R.; Watkin, D.J.; Thompson, A.L.; Hogarth, G.; Wilton-Ely, J.D.E.T. Multimetallic arrays: Symmetrical bi-, tri- and tetrametallic complexes based on the group 10 metals and the functionalisation of gold nanoparticles with nickel-phosphine surface units. Dalton Trans. 2009, 3688-3697. [CrossRef]

102. Oliver, K.; White, A.J.P.; Hogarth, G.; Wilton-Ely, J.D.E.T. Multimetallic complexes of group 10 and 11 metals based on polydentate dithiocarbamate ligands. Dalton Trans. 2011, 40, 5852-5864. [CrossRef]

103. Khan, H.; Badshah, A.; Zia-ur-Rehman; Said, M.; Murtaza, G.; Shah, A.; Butler, I.S.; Ahmed, S.; Fontaine, F.-G. New dimeric and supramolecular mixed ligand palladium(II) dithiocarbamates as potent DNA binders. Polyhedron 2012, 39, 1-8. [CrossRef] 
104. Prakasam, B.A.; Lahtinen, M.; Peuronen, A.; Muruganandham, M.; Kolehmainen, E.; Haapaniemi, E.; Sillanpaa, M. Synthesis, NMR spectral and structural studies on mixed ligand complexes of Pd(II) dithiocarbamates: First structural report on palladium(II) dithiocarbamate with SCN ${ }^{-}$ligand. J. Mol. Struct. 2016, 1108, 195-202. [CrossRef]

105. Shaheen, F.; Badshah, A.; Gielen, M.; Gieck, C.; de Vos, D. Synthesis, characterization and in vitro cytotoxicity of palladium(II) complexes with mixed ligands. X-ray diffraction study of $\mathrm{C}_{31} \mathrm{H}_{36} \mathrm{ClNPPdS}_{2}$. Appl. Organomet. Chem. 2007, 21, 633-640. [CrossRef]

106. Shaheen, F.; Najam-Ul-Haq, M.; Wurst, K.; Badshah, A.; Ali, S. Chloro(4-methylpiperidine-1-dithiocarbamato- $\left.\kappa^{2} S: S^{\prime}\right)$ (triphenylphosphine-кP)palladium(II). Acta Crystallogr. Sect. E Struct. Rep. Online 2006, 62, m136-m137. [CrossRef]

107. Shaheen, F.; Badshah, A.; Gielen, M.; Croce, G.; Florke, U.; de Vos, D.; Ali, S. In vitro assessment of cytotoxicity, anti-inflammatory, antifungal properties and crystal structures of metallacyclic palladium(II) complexes. J. Organomet. Chem. 2010, 695, 315-322. [CrossRef]

108. Florke, U. Chloro-(3,4-dihydroisoquinoline-2(1H)-carbodithioato)-(tris(2-methylphenyl)phosphine)-palladium(ii). CSD Commun. 2016. [CrossRef]

109. Nawaz, H.; Waseem, A.; Nafees, M.; Arshad, M.N.; Rashid, U. Synthesis, characterization, cytotoxicity and computational studies of new phosphine and carbodithioate based palladium(II) complexes. Appl. Organomet. Chem. 2017, 31, e3771. [CrossRef]

110. Khan, S.Z.; Butler, I.S.; Belanger-Gariepy, F. New ternary palladium(II) complexes: Synthesis, characterization, in vitro anticancer and antioxidant activities. Inorg. Chem. Commun. 2019, 105, 140-146. [CrossRef]

111. Khan, S.Z.; Amir, M.K.; Ullah, I.; Aamir, A.; Pezzuto, J.M.; Kondratyuk, T.; Bélanger-Gariepy, F.; Ali, A.; Khan, S.; Zia-ur-Rehman. New heteroleptic palladium(II) dithiocarbamates: Synthesis, characterization, packing and anticancer activity against five different cancer cell lines. Appl. Organomet. Chem. 2016, 30, 392-398. [CrossRef]

112. Khan, S.Z.; Amir, M.K.; Abbasi, R.; Tahir, M.N.; Zia-ur-Rehman. New 3D and 2D supramolecular heteroleptic palladium(II) dithiocarbamates as potent anticancer agents. J. Coord. Chem. 2016, 69, 2999-3009. [CrossRef]

113. Khan, S.Z.; Amir, M.K.; Naseer, M.M.; Abbasi, R.; Mazhar, K.; Tahir, M.N.; Awan, I.Z.; Zia-ur-Rehman. Heteroleptic Pd(II) dithiocarbamates: Synthesis, characterization, packing and in vitro anticancer activity against HeLa cell line. J. Coord. Chem. 2015, 68, 2539-2551. [CrossRef]

114. Khan, S.Z.; Amir, M.K.; Ullah, I.; Akhter, M.S.; Bélanger-Gariepy, F. Heteroleptic palladium(II) dithiocarbamates: Synthesis, characterization and in vitro biological screening. J. Mol. Struct. 2018, 1156, 564-570. [CrossRef]

115. Khan, H.; Badshah, A.; Said, M.; Murtaza, G.; Ahmad, J.; Jean-Claude, B.J.; Todorova, M.; Butler, I.S. Anticancer metallopharmaceutical agents based on mixed-ligand palladium(II) complexes with dithiocarbamates and tertiary organophosphine ligands. Appl. Organomet. Chem. 2013, 27, 387-395. [CrossRef]

116. Khan, H.; Badshah, A.; Murtaz, G.; Said, M.; Neuhausen, C.; Todorova, M.; Jean-Claude, B.J.; Butler, I.S. Synthesis, characterization and anticancer studies of mixed ligand dithiocarbamate palladium(II) complexes. Eur. J. Med. Chem. 2011, 46, 4071-4077. [CrossRef]

117. Khan, H.; Badshah, A.; Said, M.; Murtaza, G.; Sirajuddin, M.; Ahmad, J.; Butler, I.S. Synthesis, structural characterization and biological screening of heteroleptic palladium(II) complexes. Inorg. Chim. Acta 2016, 447, 176-182. [CrossRef]

118. Chan, E.Y.Y.; Zhang, Q.-F.; Sau, Y.-K.; Lo, S.M.F.; Sung, H.H.Y.; Williams, I.D.; Haynes, R.K.; Leung, W.-H. Chiral bisphosphinite metalloligands derived from a P-chiral secondary phosphine oxide. Inorg. Chem. 2004, 43, 4921-4926. [CrossRef]

119. Serrano, J.L.; García, L.; Pérez, J.; Pérez, E.; Galiana, J.M.; García, J.; Martínez, M.; Sánchez, G.; da Silva, I. New cyclometallated precursors of unsubstituted $\mathrm{N}$-phenylpyrazole $\left[\{\mathrm{Pd}(\mathrm{phpz})(\mu-\mathrm{X})\}_{2}\right](\mathrm{X}=\mathrm{AcO}$ or $\mathrm{OH})$ and study of their reactivity towards selected ligands. Dalton Trans. 2011, 40, 156-168. [CrossRef]

120. Kondrashov, M.; Fleckhaus, A.; Gritcenko, R.; Wendt, O.F. Crystal structure of (piperidine-1-carbodithioato- $\left.\kappa^{2} S, S\right)[2-($ pyridin-2yl)phenyl- $\mathrm{k}^{2} \mathrm{C}^{1}, N$ ]palladium(II). Acta Crystallogr. Sect. E Crystallogr. Commun. 2015, 71, m166. [CrossRef] [PubMed]

121. Lentijo, S.; Miguel, J.A.; Espinet, P. Cyclopalladated complexes of perylene imine: Mononuclear complexes with five- or six-membered metallacycles. Dalton Trans. 2011, 40, 7602-7609. [CrossRef] [PubMed]

122. Cheung, K.-M.; Zhang, Q.-F.; Mak, W.-L.; Sung, H.H.Y.; Williams, I.D.; Leung, W.-H. Bimetallic complexes with porphyrins containing a cyclometalated phenylpyridine group. J. Organomet. Chem. 2005, 690, 253-260. [CrossRef]

123. Schildbach, D.; Arroyo, M.; Lehmen, K.; Martin-Barrios, S.; Sierra, L.; Villafane, F.; Strohmann, C. [(Piperidinomethyl)silylmethyl] cyclopalladated complexes: Their synthesis, reactivity, and solid state structures. Organometallics 2004, 23, 3228-3238. [CrossRef]

124. Wang, H.-F.; Yih, K.-H.; Leeb, G.-H.; Huang, S.-L. Synthesis, reactivity and crystal structures of the Palladium(II) complexes with the pyrimidine containing ligand: Crystal structures of $\left[\mathrm{Pd}\left(\mathrm{PPh}_{3}\right)\left(\eta^{1}-\mathrm{C}_{4} \mathrm{H}_{3} \mathrm{~N}_{2}\right)\left(\eta^{2}-\mathrm{S}_{2} \mathrm{CNC}_{4} \mathrm{H}_{8}\right)\right]$ and $\left[\mathrm{Pd}\left(\mathrm{PPh}_{3}\right)\left(\eta^{1}-\mathrm{C}_{4} \mathrm{H}_{3} \mathrm{~N}_{2}\right)\left(\eta^{2}-\right.\right.$ Tp)]. J. Chin. Chem. Soc. 2011, 58, 174-180. [CrossRef]

125. Kwok, W.-H.; Lu, G.-L.; Rickard, C.E.F.; Roper, W.R.; Wright, L.J. The structurally characterised silyl complexes, $\left.\mathrm{Os}_{\left(\kappa^{2}-\mathrm{S}_{2} \mathrm{CNMe}\right.}\right)^{-}$ $\left(\mathrm{SiMeCl}_{2}\right)(\mathrm{CO})\left(\mathrm{PPh}_{3}\right)_{2}$ and $\mathrm{Os}\left(\mathrm{\kappa}^{2}-\mathrm{S} 2 \mathrm{CNMe} 2\right)\left(\mathrm{SiCl}_{3}\right)(\mathrm{CO})\left(\mathrm{PPh}_{3}\right)_{2}$, which have remarkably unreactive Si-Cl bonds. J. Organomet. Chem. 2006, 691, 2593-2598. [CrossRef]

126. Albrecht, M.; Kwok, W.-H.; Lu, G.-L.; Rickard, C.E.F.; Roper, W.R.; Salter, D.M.; Wright, L.J. Osmadisiloxane and osmastannasiloxane complexes derived from silanolate complexes of osmium(II). Inorg. Chim. Acta 2005, 358, 1407-1419. [CrossRef]

127. Möhlen, M.M.; Rickard, C.E.F.; Roper, W.R.; Whittell, G.R.; Wright, L.J. Syntheses, reactions, and structures of osmium(II) stannyl complexes with the simple stannyl ligands $-\mathrm{SnH}_{3},-\mathrm{SnH}_{2} \mathrm{Me}$, and $-\mathrm{SnHMe}$. Inorg. Chim. Acta 2007, 360, 1287-1297. [CrossRef] 


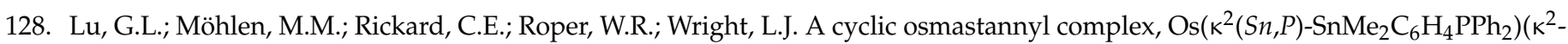
$\left.\mathrm{S}_{2} \mathrm{CNMe}_{2}\right)(\mathrm{CO})\left(\mathrm{PPh}_{3}\right)$ derived from the osmastannol complex, $\mathrm{Os}\left(\mathrm{SnMe}{ }_{2} \mathrm{OH}\right)\left(\kappa^{2}-\mathrm{S}_{2} \mathrm{CNMe}_{2}\right)(\mathrm{CO})\left(\mathrm{PPh}_{3}\right)_{2}$. Inorg. Chim. Acta 2005, 358, 4145-4155. [CrossRef]

129. Möhlen, M.M.; Rickard, C.E.F.; Roper, W.R.; Whittell, G.R.; Wright, L.J. Syntheses, reactions, and structures of osmium(II) distannyl complexes, $\mathrm{L}_{n} \mathrm{Os}-\mathrm{SnMe}_{2} \mathrm{SnR}_{3}(\mathrm{R}=\mathrm{Me}, \mathrm{Ph})$, from reaction between $\mathrm{L}_{n} \mathrm{Os}-\mathrm{SnClMe}_{2}$ and either $\mathrm{LiSnMe}_{3}$ or $\mathrm{KSnPh}_{3}$. J. Organomet. Chem. 2006, 691, 4065-4075. [CrossRef]

130. Karim, M.M.; Abser, M.N.; Hassan, M.R.; Ghosh, N.; Alt, H.G.; Richards, I.; Hogarth, G. Oxidative-addition of thiuram disulfides to osmium(0): Synthesis of cis-[Os(CO) $\left.)_{2}\left(\mathrm{~S}_{2} \mathrm{CNR}_{2}\right)_{2}\right]\left(\mathrm{R}=\mathrm{Me}, \mathrm{Et}, \mathrm{Cy}, \mathrm{CH}_{2} \mathrm{CH}_{2} \mathrm{OMe}\right)$ and molecular structures of cis- $\left[\mathrm{Os}(\mathrm{CO})_{2}\left(\mathrm{~S}_{2} \mathrm{CNMe}_{2}\right)_{2}\right]$ and $\left[\left(\mathrm{MeOCH}_{2} \mathrm{CH}_{2}\right)_{2} \mathrm{NCS}_{2}\right.$. Polyhedron 2012, 42, 84-88. [CrossRef]

131. Bhoumik, N.C.; Saha, T.K.; Ghosh, S.; Nesterov, V.N.; Richmond, M.G.; Kabir, S.E. Facile Os-Os bond cleavage in the reactions of $\left[\mathrm{Os}_{3}(\mathrm{CO})_{10}(\mathrm{NCMe})_{2}\right]$ and $\left[\mathrm{Os}_{3}(\mathrm{CO})_{10}(\mu-\mathrm{H})_{2}\right]$ with tetramethylthiuram disulfide (tmtd): Syntheses and crystal structures of new polynuclear osmium carbonyl complexes containing a dimethyldithiocarbamate ligand(s). J. Organomet. Chem. 2020, $911,121133$. [CrossRef]

132. Garcia-Orozco, I.; Pastor, C.J.; Souto, B.; Delgado, E.; Hernandez, E.; Alvarez-Toledano, C. Nonacarbonyl- $\mu_{3}-N, N-$ diethyldithiocarbamato- ${ }^{3} S: S: S^{\prime}-\mu$-hydrido-triosmium(3 Os-Os). Acta Crystallogr. Sect. E Crystallogr. Commun. 2005, 61, m1644-m1645. [CrossRef]

133. Chen, L.-Q. ( $N, N^{\prime}$-Diethyldithiocarbamato- $\left.\kappa^{2} S, S^{\prime}\right)$ bis[2-(2-pyridyl)phenyl-k $\left.{ }^{2} C^{1}, N\right]$ iridium(III). Acta Crystallogr. Sect. E Crystallogr. Commun. 2007, 63, m2078. [CrossRef]

134. Lau, M.-K.; Cheung, K.-M.; Zhang, Q.-F.; Song, Y.; Wong, W.-T.; Williams, I.D.; Leung, W.-H. Iridium(III) and rhodium(III) cyclometalated complexes containing sulfur and selenium donor ligands. J. Organomet. Chem. 2004, 689, 2401-2410. [CrossRef]

135. Wang, X.-M.; Qiang, J.-Y.; Jia, A.-Q.; Tong, B.; Zhang, Q.-F. Syntheses, crystal structures and phosphorescence properties of cyclometalated iridium(III) bis(pyridylbenzaldehyde) complexes with dithiolate ligands. Z. Naturforsch. B Chem. Sci. 2017, 72, 941-946. [CrossRef]

136. Lu, G.-Z.; Tu, Z.-L.; Liu, L.; Zhang, W.-W.; Zheng, Y.-X. Fast synthesis of iridium (iii) complexes with sulfur-containing ancillary ligand for high-performance green OLEDs with EQE exceeding 31\%. J. Mater. Chem. C 2019, 7, 7273-7278. [CrossRef]

137. Lu, G.-Z.; Wu, R.; Liu, L.; Zhou, L.; Zheng, Y.-X.; Zhang, W.-W.; Zuo, J.-L.; Zhang, H. A series of red iridium(III) complexes using flexible dithiocarbamate derivatives as ancillary ligands for highly efficient phosphorescent OLEDs. Mater. Chem. Front. 2019, 3, 860-866. [CrossRef]

138. Lu, G.Z.; Su, N.; Yang, H.Q.; Zhu, Q.; Zhang, W.-W.; Zheng, Y.-X.; Zhou, L.; Zuo, J.-L.; Chen, Z.-X.; Zhang, H.-J. Rapid room temperature synthesis of red iridium(iii) complexes containing a four-membered Ir-S-C-S chelating ring for highly efficient OLEDs with EQE over 30\%. Chem. Sci. 2019, 10, 3535-3542. [CrossRef] [PubMed]

139. Su, N.; Li, F.-L.; Zheng, Y.-X. Four-membered red iridium(III) complexes with Ir-S-C-S structures for efficient organic lightemitting diodes. J. Mater. Chem. C 2020, 8, 7411-7416. [CrossRef]

140. Lu, G.-Z.; Li, X.; Liu, L.; Zhou, L.; Zheng, Y.-X.; Zhang, W.-W.; Zuo, J.-L.; Zhang, H. Efficient phosphorescent red iridium(III) complexes containing a four-membered Ir-S-C-S ring backbone and large hindered spacers for high-performance OLEDs. J. Mater. Chem. C 2019, 7, 3862-3868. [CrossRef]

141. Mei, Q.; Chen, C.; Tian, R.; Yang, M.; Tong, B.; Hua, Q.; Shi, Y.; Fan, Q.; Ye, S. Highly efficient orange phosphorescent organic light-emitting diodes based on an iridium(III) complex with diethyldithiocarbamate ( $\mathrm{S}^{\wedge} \mathrm{S}$ ) as the ancillary ligand. RSC Adv. 2016, 6, 64003-64008. [CrossRef]

142. Tong, B.; Ma, P.; Zhang, M.; Liu, Y.; Mei, Q.; Zhang, Q.-F. Phosphorescent iridium(III) carbodithioate complex for the detection of $\mathrm{Hg}^{2+}$ and acetonitrile. Inorg. Chem. Commun. 2013, 37, 121-126. [CrossRef]

143. Katlenok, E.A.; Zolotarev, A.A.; Ivanov, A.Y.; Smirnov, S.N.; Baichurin, R.I.; Balashev, K.P. Complexes of Ir(III) and Pt(II) with cyclometallated 2-phenylbenzothiazole and chelating diethyldithiocarbamate and o-ethyldithiocarbonate ions: Structures and optical and electrochemical properties. Russ. J. Coord. 2016, 42, 178-186. [CrossRef]

144. Katlenok, E.A.; Zolotarev, A.A.; Balashev, K.P. Influence of ligand donor-acceptor properties on the structure, optical, and electrochemical characteristics of $\mathrm{Ir}(\mathrm{III})$ complexes with cyclometallated 2-phenylbenzotiazole. Russ. J. Gen. Chem. 2016, 86, 2508-2514. [CrossRef]

145. Chen, L.; Wu, Z.; Yang, J.; Zhang, S. The synthesis and crystal structure of bis(2-(benzo[d]thiazol-2-yl)-5-methylbenzen-1-ido$\left.\kappa^{2} C, N\right)-\left(\mathrm{N}, \mathrm{N}^{\prime}\right.$-diethyldithiocarbamato-k $\left.{ }^{2} S, S^{\prime}\right)$ iridium(III), $\mathrm{C}_{33} \mathrm{H}_{30} \mathrm{~N}_{3} \mathrm{~S}_{4}$ Ir. Z. Kristallogr. New Cryst. Struct. 2019, 234, 1173-1176. [CrossRef]

146. Chen, L.; Yang, C.; Qin, J.; Gao, J.; You, H.; Ma, D. Synthesis, structure, electrochemistry, photophysics and electroluminescence of 1,3,4-oxadiazole-based ortho-metalated iridium(III) complexes. J. Organomet. Chem. 2006, 691, 3519-3530. [CrossRef]

147. Chen, L.; Yang, C.; Li, M.; Qin, J.; Gao, J.; You, H.; Ma, D. Supramolecular architectures, photophysics, and electroluminescence of 1,3,4-oxadiazole-based iridium(III) complexes: From $\mu$-dichloro bridged dimer to mononuclear complexes. Cryst. Growth Des. 2007, 7, 39-46. [CrossRef]

148. Clark, G.R.; Ferguson, L.A.; McIntosh, A.E.; Söhnel, T.; Wright, L.J. Functionalization of metallabenzenes through nucleophilic aromatic substitution of hydrogen. J. Am. Chem. Soc. 2010, 132, 13443-13452. [CrossRef]

149. Scharwitz, M.; van Almsick, T.; Sheldrick, W.S. Carbonyl(diethyldithioarbamato)( $\eta^{5}$-pentamethylcyclopentadienyl)iridium(III) trifluoromethanesulfonate. Acta Crystallogr. Sect. E Crystallogr. Commun. 2007, E63, m105-m107. [CrossRef] 
150. Scharwitz, M.; Oppel, I.M.; Sheldrick, W.S. Bis( $\mu$-diethyldithiocarbamato)bis[( $\eta^{5}$-pentamethylcyclopentadienyl)iridium(III)] bis(perchlorate). Acta Crystallogr. Sect. E Crystallogr. Commun. 2007, 63, m2065-m2066. [CrossRef]

151. Kotera, M.; Sekioka, Y.; Suzuki, T. Structural versatility of 5-methyltetrazolato complexes of ( $\eta^{5}$-pentamethylcyclopentadienyl) iridium(III) incorporating 2,2'-bipyridine, $N, N$-dimethyldithiocarbamate, or 2-pyridinethiolate ligands. Inorg. Chem. 2008, 47, 3498-3508. [CrossRef] [PubMed]

152. Shaver, A.; Mouatassim, B.E.; Mortini, F.; Belanger-Gariepy, F.; Lough, A. Reactions of $\left(\eta^{5}-\mathrm{C}_{5} \mathrm{Me}_{5}\right) \operatorname{Ir}\left(\mathrm{PMe}_{3}\right)\left(\mathrm{SH}_{2}\right.$ and $\left(\eta^{5}-\right.$ $\left.\mathrm{C}_{5} \mathrm{Me}_{5}\right) \operatorname{Ir}\left(\mathrm{PMe}_{3}\right)(\mathrm{SH})(\mathrm{H})$ with thionylaniline (PhNSO) to give novel iridium $\mathrm{S}_{3} \mathrm{O}$ and $\mathrm{S}_{2} \mathrm{O}$ complexes. Organometallics 2007, 26, 4229-4233. [CrossRef]

153. Ariyoshi, K.; Suzuki, T.; Mayer, J.M.; Kojima, M. Reactivities of the $\mathrm{N}$-atom-inserted ligands, $\mathrm{NSC}\left(\mathrm{NR}_{2}\right) \mathrm{S}^{2-}$ and $\mathrm{SN}^{-} \mathrm{C}\left(\mathrm{NR}_{2}\right) \mathrm{S}^{2-}$, in iridium(III) complexes. Chem. Lett. 2011, 40, 831-833. [CrossRef]

154. Onwudiwe, D.C.; Mugo, J.N.; Hrubaru, M.; Hosten, E. Bis diallyl dithiocarbamate Pt(II) complex: Synthesis, characterization, thermal decomposition studies, and experimental and theoretical studies on its crystal structure. J. Sulfur Chem. 2015, 36, 36-47. [CrossRef]

155. Zaeva, A.S.; Ivanov, M.A.; Gerasimenko, A.V.; Ivanov, A.V.; Antzutkin, O.N. Dialkyldithiocarbamate platinum(II) complexes of $\left[\mathrm{Pt}\left(\mathrm{S}_{2} \mathrm{CNR}_{2}\right)_{2}\right]\left(\mathrm{R}=\right.$ iso- $\mathrm{C}_{3} \mathrm{H}_{7}$, iso- $\left.\mathrm{C}_{4} \mathrm{H}_{9}\right)$ : Preparation, ${ }^{13} \mathrm{C} \mathrm{CP}-\mathrm{MAS} \mathrm{NMR}$, molecular structure, supramolecular self-assembly and thermal behaviour. Polyhedron 2020, 175, 114166. [CrossRef]

156. Meletov, K.P.; Konarev, D.V. Raman study of the pressure-induced phase transitions in the molecular donor-acceptor complex $\left\{\mathrm{Pt}(\mathrm{dbdtc})_{2}\right\} \mathrm{C}_{60}$. Chem. Phys. Lett. 2012, 553, 21-25. [CrossRef]

157. Ehsan, M.A.; Younas, M.; Rehman, A.; Altaf, M.; Khan, M.Y.; Al-Ahmed, A.; Ahmad, S.; Isab, A.A. Synthesis and utilization of platinum(II) dialkyldithiocarbamate precursors in aerosol assisted chemical vapor deposition of platinum thin films as counter electrodes for dye-sensitized solar cells. Polyhedron 2019, 166, 186-195. [CrossRef]

158. Poirier, S.; Rahmani, F.; Reber, C. Large d-d luminescence energy variations in square-planar bis(dithiocarbamate) platinum(II) and palladium(II) complexes with near-identical $\mathrm{MS}_{4}$ motifs: A variable-pressure study. Dalton Trans. 2017, 46, 5279-5287. [CrossRef]

159. Poirier, S.; Roberts, R.J.; Le, D.; Leznoff, D.B.; Reber, C. Interpreting effects of structure variations induced by temperature and pressure on luminescence spectra of platinum(II) bis(dithiocarbamate) compounds. Inorg. Chem. 2015, 54, 3728-3735. [CrossRef] [PubMed]

160. Leka, Z.; Vojta, D.; Kosovic, M.; Latinovic, N.; Dakovic, M.; Visnjevac, A. Syntheses, structures and antifungal activities of novel Co, Mo and Pt complexes with triammonium N,N-diacetatedithiocarbamate. Polyhedron 2014, 80, 233-242. [CrossRef]

161. Liebing, P.; Witzorke, J.; Oehler, F.; Schmeide, M. Dithiocarbamatocarboxylate (DTCC) ligands-building blocks for hard/softheterobimetallic coordination polymers. Inorg. Chem. 2020, 59, 2825-2832. [CrossRef]

162. Ajibade, P.A.; Fatokun, A.A.; Andrew, F.P. Synthesis, characterization and anti-cancer studies of $\mathrm{Mn}$ (II), $\mathrm{Cu}(\mathrm{II}), \mathrm{Zn}(\mathrm{II})$ and Pt(II) dithiocarbamate complexes-crystal structures of the $\mathrm{Cu}(\mathrm{II})$ and $\mathrm{Pt}$ (II) complexes. Inorg. Chim. Acta 2020, 504, 119431. [CrossRef]

163. Andrew, F.P.; Ajibade, P.A. Synthesis, characterization and anticancer studies of bis(1-phenylpiperazine dithiocarbamato) $\mathrm{Cu}(\mathrm{II})$, $\mathrm{Zn}(\mathrm{II})$ and $\mathrm{Pt}(\mathrm{II})$ complexes: Crystal structures of 1-phenylpiperazine dithiocarbamato-S,S' zinc(II) and Pt(II). J. Mol. Struct. 2018, 1170, 24-29. [CrossRef]

164. Ivanov, M.A.; Zaeva, A.S.; Gerasimenko, A.V.; Ivanov, A.V. Platinum(II) cyclo-hexamethylenedithiocarbamate complex, $\left[\mathrm{Pt}\left\{\mathrm{S}_{2} \mathrm{CN}\left(\mathrm{CH}_{2}\right)_{6}\right\}_{2}\right]$ and its solvated form, $\left[\mathrm{Pt}\left\{\mathrm{S}_{2} \mathrm{CN}\left(\mathrm{CH}_{2}\right)_{6}\right\}_{2}\right] \cdot \mathrm{CHCl}_{3}$ : Crystal and molecular structures, ${ }^{13} \mathrm{C} \mathrm{CP} / \mathrm{MAS} \mathrm{NMR}$ data, and thermal behavior. Russ. J. Coord. Chem. 2013, 39, 764-770. [CrossRef]

165. Keter, F.K.; Guzei, I.A.; Darkwa, J. N-heterocyclic dithiocarbamate platinum(II) complexes: Unexpected transformation of dithiocarbamate to oxodithiocarbonate in phosphinoplatinum complexes in solution. Inorg. Chem. Commun. 2013, $27,60-63$. [CrossRef]

166. Montagner, D.; Miguel, P.J.S. Unique $\mathrm{Pt}_{5}$ metallacycle: $\left[\mathrm{Pt}{ }^{\mathrm{II}} \mathrm{Cl}(\text { pyrrolidinedithiocarbamate) }]_{5}\right.$. Dalton Trans. 2011, 40, 10809-10811. [CrossRef]

167. Isab, A.A.; Ali, M.A.J.; Sharif, S.; Khan, I.U.; Kang, S.K.; Khalid, T.; Saleem, M.; Ahmad, S. Synthesis, crystal structure and antimicrobial studies of chlorido(dimethylsulfoxide- $\mathrm{K} S$ )(pyrrolidinedithiocarbamato- $\mathrm{K}^{2} \mathrm{~S}, \mathrm{~S}^{\prime}$ ) platinum(II). Inorg. Chem. Commun. 2011, 14, 1962-1965. [CrossRef]

168. Rheingold, A.L.; Reger, D.L. Chloro-(diethylcarbamodithioato)-(triethylphosphine)-platinum. CSD Commun. 2019. [CrossRef]

169. Amir, M.K.; Hayat, F.; Khan, S.Z.; Hogarth, G.; Kondratyuk, T.; Pezzuto, J.M.; Tahir, M.N. Monofunctional platinum(II) dithiocarbamate complexes: Synthesis, characterization and anticancer activity. RSC Adv. 2016, 6, 110517-110524. [CrossRef]

170. Imran, M.; Kondratyuk, T.; Belanger-Gariepy, F. New ternary platinum(II) dithiocarbamates: Synthesis, characterization, anticancer, DNA binding and DNA denaturing studies. Inorg. Chem. Commun. 2019, 103, 12-20. [CrossRef]

171. Verron, R.; Achard, T.; Seguin, C.; Fournel, S.; Bellemin-Laponnaz, S. Synthesis and characterization of n-heterocyclic carbene dithiocarbamate platinum complexes with antitumoral activity. Eur. J. Inorg. Chem. 2020, 2020, 2552-2557. [CrossRef]

172. Forniés, J.; Sicilia, V.; Casas, J.M.; Martín, A.; López, J.A.; Larraz, C.; Borja, P.; Ovejero, C. Pt-Ag clusters and their neutral mononuclear Pt(II) starting complexes: Structural and luminescence studies. Dalton Trans. 2011, 40, 2898-2912. [CrossRef]

173. Expósito, J.E.; Álvarez-Paíno, M.; Aullón, G.; Miguel, J.A.; Espinet, P. Higher fluorescence in platinum(IV) orthometallated complexes of perylene imine compared with their platinum(II) or palladium(II) analogues. Dalton Trans. 2015, 44, 16164-16176. [CrossRef] 
174. Paziresh, S.; Aghakhanpour, R.B.; Fuertes, S.; Sicilia, V.; Hosseini, F.N.; Nabavizadeh, S.M. A double rollover cycloplatinated(II) skeleton: A versatile platform for tuning emission by chelating and non-chelating ancillary ligand systems. Dalton Trans. 2019, 48, 5713-5724. [CrossRef]

175. Rheingold, A.L.; Garza, D. (Diethylcarbamodithioato)-(isobutyl)-(triethylphosphine)-platinum(II). CSD Commun. 2015. [CrossRef]

176. Burt, L.K.; Hill, A.F. Heterobimetallic $\mu_{2}$-carbido complexes of platinum and tungsten. Dalton Trans. 2020, $49,8143-8161$. [CrossRef]

177. Fortuño, C.; Martín, A.; Mastrorilli, P.; Latronico, M.; Petrelli, V.; Todisco, S. Stable mixed-valence diphenylphosphanido bridged platinum(II)-platinum(IV) complexes. Dalton Trans. 2020, 49, 4935-4955. [CrossRef]

178. Heard, P.J.; Kite, K.; Nielsen, J.S.; Tocher, D.A. Trimethylplatinum(IV) complexes of dithiocarbamato ligands: An experimental NMR study on the barrier to C-N bond rotation. J. Chem. Soc. Dalton Trans. 2000, 1349-1356. [CrossRef]

179. Evans, D.F. The determination of the paramagnetic susceptibility of substances in solution by nuclear magnetic resonance. J. Chem. Soc. 1959, 2003-2005. [CrossRef]

180. Brustolin, L.; Nardon, C.; Pettenuzzo, N.; Zuin Fantoni, N.; Quarta, S.; Chiara, F.; Gambalunga, A.; Trevisan, A.; Marchiò, L.; Pontisso, P.; et al. Synthesis, chemical characterization and cancer cell growth-inhibitory activities of $\mathrm{Cu}$ (II) and Ru(III) aliphatic and aromatic dithiocarbamato complexes. Dalton Trans. 2018, 47, 15477-15486. [CrossRef] [PubMed]

181. Robertson, D.R.; Stephenson, T.A. Metal complexes of sulphur ligands. Part 15. Reaction of bis[( $\eta$-arene)dichlororuthenium] and bis[dichloro( $\eta$-pentamethylcyclopentadienyl)metal] complexes of rhodium and iridium with various dithioacid ligands. J. Chem. Soc. Dalton Trans. 1978, 486-495. [CrossRef]

182. Forth, C.S. Studies on Sulfur-Capped Osmium and Ruthenium Clusters with Focus on Dynamics; University of London: London, UK, 2003.

183. Van Gaal, H.L.M.; Diesveld, J.W.; Pijpers, F.W.; Van der Linden, J.G.M. Carbon-13 NMR spectra of dithiocarbamates. Chemical shifts, carbon-nitrogen stretching vibration frequencies and. pi.-bonding in the NCS2 fragment. Inorg. Chem. 1979, 18, 3251-3260. [CrossRef]

184. Ursini, C.V. Platinum-195 nuclear magnetic resonance of organometallic compounds. Quím. Nova 1997, 20, 72-82. [CrossRef]

185. Trzaska, S. Cisplatin. Chem. Eng. News 2005, 83, 52. [CrossRef]

186. Kamoon, R.A.; Nadhum, S.A.; Mohammed, M.H. Dithiocarbamates derivatives as anticancer agents: A Review. Ann. Trop. Med. Public Health 2020, 23, S19. [CrossRef]

187. Moharana, P.; Ghosh, D.; Paira, R. Drive to organoruthenium and organoiridium complexes from organoplatinum: Nextgeneration anticancer metallotherapeutics. Inorg. Chem. Commun. 2021, 124, 108364. [CrossRef]

188. Hussain, S.; Bukhari, I.H.; Ali, S.; Shahzadi, S.; Shahid, M.; Munawar, K.S. Synthesis and spectroscopic and thermogravimetric characterization of heterobimetallic complexes with $\mathrm{Sn}(\mathrm{IV})$ and $\mathrm{Pd}(\mathrm{II})$; DNA binding, alkaline phosphatase inhibition and biological activity studies. J. Coord. Chem. 2015, 68, 662-677. [CrossRef]

189. Hussain, S.; Ali, S.; Shahzadi, S.; Shahid, M.; Tahir, A.A.; Abbas, S.M.; Riaz, M.; Ahmad, I.; Hussain, I. Multinuclear (Sn/Pd) complexes with disodium 2,2'-(dithiocarboxyazanediyl)diacetate hydrate; Synthesis, characterization and biological activities. J. Coord. Chem. 2017, 70, 4070-4092. [CrossRef]

190. Odularu, A.T.; Ajibade, P.A.; Mbese, J.Z.; Oyedeji, O.O. Developments in platinum-group metals as dual antibacterial and anticancer agents. J. Chem. 2019, 2019, 5459461. [CrossRef]

191. Anant, R.; Kapdi, A.R.; Fairlamb, I.J.S. Anti-cancer palladium complexes: A focus on $\mathrm{PdX}_{2} \mathrm{~L}_{2}$, palladacycles and related complexes. Chem. Soc. Rev. 2014, 43, 4751-4777. [CrossRef]

192. Fanelli, M.; Formica, M.; Fusi, V.; Giorgi, L.; Micheloni, M.; Paoli, P. New trends in platinum and palladium complexes as antineoplastic agents. Coord. Chem. Rev. 2016, 310, 41-79. [CrossRef]

193. Alam, M.N.; Huq, F. Comprehensive review on tumour active palladium compounds and structure-activity relationships. Coord. Chem. Rev. 2016, 316, 36-67. [CrossRef]

194. Amir, M.K.; Zebkhan, S.; Hayat, F.; Hassan, A.; Butler, I.S.; Zia-ur-Rehman. Anticancer activity, DNA-binding and DNAdenaturing aptitude of palladium(II) dithiocarbamates. Inorg. Chim. Acta 2016, 451, 31-40. [CrossRef]

195. Kang, M.S.; Choi, E.K.; Choi, D.H.; Ryu, S.Y.; Lee, H.H.; Kang, H.C.; Koh, J.T.; Kim, O.S.; Hwang, Y.C.; Yoon, S.J.; et al. Antibacterial activity of pyrrolidine dithiocarbamate. FEMS Microbiol. Lett. 2008, 280, 250-254. [CrossRef]

196. Choi, E.K.; Lee, H.H.; Kang, M.S.; Kim, B.G.; Lim, H.S.; Kim, S.M.; Kang, I.C. Potentiation of bacterial killing activity of zinc chloride by pyrrolidine dithiocarbamate. J. Microbiol. 2010, 48, 40-43. [CrossRef]

197. Yeo, C.I.; Tiekink, E.R.T.; Chew, J. Insights into the Antimicrobial Potential of Dithiocarbamate Anions and Metal-Based Species. Inorganics 2021, 9, 48. [CrossRef]

198. Giovagnini, L.; Marzano, C.; Bettio, F.; Fregona, D. Mixed complexes of $\mathrm{Pt}(\mathrm{II})$ and $\mathrm{Pd}(\mathrm{II})$ with ethylsarcosinedithiocarbamate and 2-/3-picoline as antitumor agents. J. Inorg. Biochem. 2005, 99, 2139-2150. [CrossRef] [PubMed]

199. Malik, M.A.; Afzaal, M.; O’Brien, P. Precursor Chemistry for Main Group Elements in Semiconducting Materials. Chem. Rev. 2010, 110, 4417-4446. [CrossRef] [PubMed]

200. Knapp, C.E.; Carmalt, C.J. Solution based CVD of main group materials. Chem. Soc. Rev. 2016, 45, 1036-1064. [CrossRef] [PubMed]

201. Fredrick, G.; Erling, R. On the sulfides, selenides, and tellurides of palladium. Acta Chem. Scand. 1956, 10, 1620-1634. [CrossRef] 